\title{
The Condercet paradox revisited
}

Citation for published version (APA):

Herings, P. J. J., \& Houba, H. (2010). The Condercet paradox revisited. METEOR, Maastricht University School of Business and Economics. METEOR Research Memorandum No. 009

https://doi.org/10.26481/umamet.2010009

Document status and date:

Published: 01/01/2010

DOI:

10.26481/umamet.2010009

Document Version:

Publisher's PDF, also known as Version of record

\section{Please check the document version of this publication:}

- A submitted manuscript is the version of the article upon submission and before peer-review. There can be important differences between the submitted version and the official published version of record.

People interested in the research are advised to contact the author for the final version of the publication, or visit the DOI to the publisher's website.

- The final author version and the galley proof are versions of the publication after peer review.

- The final published version features the final layout of the paper including the volume, issue and page numbers.

Link to publication

\footnotetext{
General rights rights.

- You may freely distribute the URL identifying the publication in the public portal. please follow below link for the End User Agreement:

www.umlib.nl/taverne-license

Take down policy

If you believe that this document breaches copyright please contact us at:

repository@maastrichtuniversity.nl

providing details and we will investigate your claim.
}

Copyright and moral rights for the publications made accessible in the public portal are retained by the authors and/or other copyright owners and it is a condition of accessing publications that users recognise and abide by the legal requirements associated with these

- Users may download and print one copy of any publication from the public portal for the purpose of private study or research.

- You may not further distribute the material or use it for any profit-making activity or commercial gain

If the publication is distributed under the terms of Article $25 \mathrm{fa}$ of the Dutch Copyright Act, indicated by the "Taverne" license above, 


\section{Maastricht University}

P. J ean-J acques Herings, Harold Houba

The Condercet Paradox Revisited

$\mathrm{RM} / 10 / 009$

\section{METEOR}

Maastricht University School of Business and Economics Maastricht Research School of Economics

of Technology and Organization

P.O. Box 616

NL - 6200 MD Maastricht

The Netherlands 


\title{
The Condercet Paradox Revisited*
}

\author{
P. Jean-Jacques Herings ${ }^{\dagger} \quad$ Harold Houba ${ }^{\ddagger}$
}

March 1, 2010

\begin{abstract}
We analyze the simplest Condorcet cycle with three players and three alternatives within a strategic bargaining model with recognition probabilities and costless delay. Mixed consistent subgame perfect equilibria exist whenever the geometric mean of the agents' risk coefficients, ratios of utility differences between alternatives, is at most one. Equilibria are generically unique, Pareto efficient, and ensure agreement within finite expected time. Agents propose best or second-best alternatives. Agents accept best alternatives, may reject second-best alternatives with positive probability, and reject otherwise. For symmetric recognition probabilities and risk coefficients below one, agreement is immediate and each agent proposes his best alternative.
\end{abstract}

Keywords: Bargaining, Condorcet Paradox, Consistent Subgame Perfect Equilibrium, Risk Aversion, Compromise Prone

JEL CODES: C73, C78, D72

*The authors thank Frans Spinnewyn, Peter Sudhölter, and Walter Trockel for valuable comments.

${ }^{\dagger}$ P.J.J. Herings, Department of Economics, Maastricht University, P.O. Box 616, 6200 MD, The Netherlands. E-mail: P.Herings@maastrichtuniversity.nl. This author would like to thank the Netherlands Organisation for Scientific Research (NWO) for financial support.

${ }^{\ddagger}$ H. Houba, Department of Econometrics and Tinbergen Institute, VU University Amsterdam, De Boelelaan 1105, 1081 HV Amsterdam, The Netherlands. Email: hhouba@feweb.vu.nl. 


\section{Introduction}

Decisions on collective choice problems are often taken by means of majority voting, and the analysis of such voting is therefore an important topic in political economy. When an alternative exists that beats every other feasible alternative in a pairwise vote, i.e. a Condorcet winner, then this will be the outcome reached for a huge variety of games that capture the underlying institution. Unfortunately, Condorcet winners may not exist and this gives rise to the Condorcet paradox in which any alternative can be reached from any other by a sequence of alternatives, where each alternative in the sequence beats the previous one by a pairwise majority vote. ${ }^{1}$ In its most simple form this paradox features three agents, three alternatives and agents' preferences such that a pairwise vote over the alternatives results in a Condorcet cycle: one pair of agents prefers the second alternative to the third alternative, another pair of agents prefers the first alternative to the second alternative, and a third pair of agents prefers the third alternative to the first alternative. Whether and how agents reach an agreement in this paradox cannot be answered. It is however the main research question of this paper after adding some additional structure to this voting situation.

We take the strategic bargaining approach to analyze the Condorcet paradox, an approach that is advocated in Banks and Duggan (2000). Such an approach has several advantages. It makes explicit how alternatives that are up for voting are selected and how agents vote on alternatives. The equilibrium strategies specify far-sighted beliefs on and off the equilibrium path regarding any future continuation. We analyze a version of Baron-Ferejohn's (1989) bargaining model with majority voting over proposed alternatives in which the division of a surplus is replaced by a discrete set of alternatives consisting of three alternatives. Preferences over these alternatives form a Condorcet cycle and perpetual disagreement is the worst outcome. In every bargaining round, exogenous and positive recognition probabilities select one agent who has the right to propose. This recognized agent either proposes one of the three alternatives or gives up the right to propose in which case bargaining proceeds to the next round. In the former case, the other agents publicly vote in a sequential order. Majority voting on three alternatives implies that one vote in favor suffices for acceptance, after which the alternative will be implemented. Otherwise, no alternative is implemented and we proceed to the next round where random selection determines the proposer. In order to stay as closely as possible to the literature on the Condorcet paradox, we refrain from discounting the future or the equivalent of some exogenous probability of permanent breakdown. Our bargaining model extends the wellknown alternating-offers model of Rubinstein (1982) and Binmore (1987) to three randomly proposing agents. This model is also closely related to the very general set-up in Banks and Duggan (2000), though the case with a discrete number of alternatives considered here is ruled out by their convexity assumption regarding the space of alternatives.

We characterize the set of mixed consistent subgame perfect equilibria. A subgame perfect equilibrium is said to be consistent (CPE) if the strategy of an agent is the same whenever the agent faces the same continuation game. This notion of consistency corresponds to the "invariance with respect to isomorphisms" of Harsanyi and Selten (1988), p. 73. This equilibrium notion is in general weaker than the notion of Markov-perfect or

\footnotetext{
${ }^{1}$ This literature includes e.g. Plott (1967), Rubinstein (1979), Schofield (1983), Cox (1984), and Le Breton (1987). The result about sequences of pairwise votes is shown in McKelvey $(1976,1979)$.
} 
stationary equilibrium as defined in Maskin and Tirole (2001), but both are closely related for the class of games under consideration. ${ }^{2}$ For a foundation of stationary equilibria, we refer to Bhaskar, Mailath, and Morris (2009).

Our main results give a novel perspective on the indeterminacy of the simplest Condorcet paradox when it is embedded in an institutional setting where a recognized agent puts up an alternative for majority voting. We discuss our main results pointwise:

Existence. We derive a very simple condition that is necessary and sufficient for the existence of a mixed CPE. To express this condition, we define an agent's risk coefficient as the ratio of the utility difference between his best and second-best alternative to the utility difference between his second-best and his third-best alternative. Risk coefficients are closely related to the risk limit of Zeuthen (1930) and Harsanyi (1977). The condition for existence states that the geometric mean of the agents' risk coefficients should be less than or equal to one. We define a compromise-prone (or risk averse) agent as someone who prefers his second-best alternative to the fair lottery over his best and worst alternative. The subclass with three compromise-prone agents obeys the condition for existence.

Agreement within finite expected time. Every mixed CPE implies a stochastic equilibrium outcome that can be seen as a lottery over all three alternatives, each with positive probability. The probability of perpetual disagreement is zero. Consequently, each agent's expected equilibrium utility lies strictly between the utility associated with his worst and best alternative. We also establish the stronger result that each agent's expected equilibrium utility is at most the utility level of the second-best alternative for sure. The main message is that, in the presence of Condorcet cycles, agents reach an agreement within finite expected time.

Pareto efficiency. Even though CPE may involve stochastic delay, such delay is costless. Since the equilibrium outcome is a lottery over the three alternatives, it follows that every CPE is Pareto efficient.

Four main cases. The equilibrium analysis distinguishes eight cases, of which seven can actually occur. Of these cases, three are degenerate and four have positive Lebesgue measure. In these last four cases, recognized agents apply a possibly degenerate lottery over their best and second-best alternative, never propose their worst alternative, and never give up the right to propose. Voters always accept for sure their best alternative, apply a possibly degenerate lottery in accepting their second-best alternative, and reject for sure their worst alternative. At most one agent proposes his second-best alternative with positive probability.

Generic uniqueness. The four main cases each admit unique CPE utilities. The three degenerate cases may admit multiple CPE utilities. Since the seven cases do not overlap, except possibly at their boundaries, the expected CPE utility is generically unique. For each of the four main cases, the unique CPE utilities are supported by unique CPE strategies, except for degenerate subcases.

Immediate agreement on the recognized agent's best alternative in one of the main cases. In one of the main cases each agent proposes for sure his best alternative, and each agent accepts for sure both his best and second-best alternatives and rejects for sure his worst alternative. Such strategies imply immediate agreement. For the symmetric recognition

\footnotetext{
${ }^{2}$ Since for convenience Maskin and Tirole (2001) restrict attention to games in which the timing of moves is independent of previous actions (p. 195), strictly speaking the notion of Markov perfect or stationary equilibrium is not defined for our bargaining model.
} 
procedure, the necessary and sufficient condition for immediate agreement is that all three agents are compromise-prone. In general, for every constellation of utilities, there exists at least one recognition procedure with immediate agreement.

No limits on stochastic delay. In the three other main cases, there is a positive probability that delay occurs. Expected delay can go to infinity when the recognition probability vector converges to a unit vector, i.e. in the limit one of the agents becomes the only recognized agent as in a bargaining model with one-sided offers.

Stochastic cycles. Infinite cycles occur according to the logic of the Condorcet paradox. However, within a cooperative game theoretic setting, Chwe (1994) argues that cycles cannot occur when agents are farsighted. We study CPE cycles in the sense of whether there is a positive probability that an equilibrium path can result in which all three alternatives have been proposed and rejected before some alternative is accepted. Such CPE cycles can occur, but only in some of the three degenerate cases.

Risk aversion improves the bargaining position. The general conclusion of the bargaining literature is that risk-aversion undermines an agent's bargaining position in two-agent bargaining models, see e.g. Roth (1985), Safra, Zhou, and Zilcha (1990), and Kihlstrom, Roth, and Schmeidler (1991). We investigate changes in risk aversion in our model. Changes in risk aversion in the main case with immediate agreement do not have any effect on the probability distribution over alternatives. For the three other main cases, a less risk-averse agent does worse in the sense that the probability of attaining his best alternative decreases, and the probabilities of attaining his second-best and worst alternatives both increase. Given Pareto efficiency, at least one agent does strictly better. We show that the equilibrium utility of one of the three agents is not affected by a change in risk aversion.

Although CPEs have a simple structure and the simplest Condorcet paradox is considered, the equilibrium analysis is rather involved because of the astonishing number of cases that can occur. These cases arise as follows. We first show that application of the CPE conditions straightforwardly implies several general properties, such as Pareto efficiency, some of the properties of CPE strategies mentioned before, and the fact that an agent's expected CPE utility is at most the utility of his second-best alternative. The latter implies four cases, each case depends upon the number of agents that exactly reach the utility level of the second-best alternative. We then show that only one of these cases is impossible, one case has two subcases, and another one even has four subcases. This makes in total the seven cases mentioned before, of which three are degenerate. For each case, we derive necessary and sufficient conditions for existence. For each case it is required that the geometric mean of the agents' risk coefficients should be at most equal to one. For each case there are additional conditions related to the recognition probabilities and the risk coefficients. We show that all additional conditions partition the parameter space associated with the common condition on the geometric mean, except for some overlap at common boundaries.

A crucial result in the analysis that helps to reduce the complexity of the problem while not discarding any essential CPEs is the following: For any CPE and triple of expected CPE utilities, there also exists a CPE that has the same triple of expected CPE utilities, no one gives up his right to propose, and in addition agent-independent rejection probabilities. It is therefore without loss of generality to investigate the latter CPEs. Then, in investigating all the cases, for each agent there are basically two unknown probabilities associated with the 
lottery by which each agent randomizes over proposing his best and second-best alternative, and the lottery by which each agent randomizes over accepting or rejecting his second-best alternative.

An important issue that we treat in a companion paper is the following. Since we analyze the model without discounting, the bargaining model lacks continuity at infinity, an issue noted by Bloch (1996) for a different bargaining model. Herings and Houba (2010) show that the one-stage-deviation principle does apply for a class of bargaining models that includes the one analyzed here and the one in Bloch (1996). Furthermore, they derive necessary and sufficient conditions under which CPEs satisfy the traditional equilibrium conditions. Loosely stated, these conditions for $\mathrm{CPE}$ require that the perperiod CPE probability of reaching some agreement is positive, which is indeed the case since perpetual disagreement cannot occur in any CPE. In this paper, we concentrate on the Condorcet paradox and we only apply this method.

The paper is organized as follows. Section 2 describes the bargaining model. Section 3 introduces the notion of CPE and characterizes the set of CPEs as the solutions to a specific system of equations. In that section, we also derive some of the general properties and reduce the complexity of the problem at hand. Then, Section 4 analyzes this system by considering the four distinct cases that are related to the number of agents that have in expectation an equilibrium utility equal to their middle alternative, where subcases are treated in subsections. Section 5 is devoted to the study of some special cases and examples. Section 6 combines all the conditions of Section 4 and studies the questions of equilibrium existence and uniqueness. Section 7 analyzes the potential for delay and cycles and Section 8 the role of risk aversion. Section 9 concludes.

\section{The Model}

We consider a decision making problem involving three agents, called players that are labeled $i=1,2,3$, and three possible outcomes, $x_{1}, x_{2}$, and $x_{3}$. We consider the case where the preferences of the players satisfy the following restriction

$$
x_{1} \succ^{1,3} x_{2} \succ^{1,2} x_{3} \succ^{2,3} x_{1}
$$

The formulation in (2.1) means that players 1 and 3 prefer the outcome $x_{1}$ to $x_{2}$, players 1 and 2 prefer the outcome $x_{2}$ to $x_{3}$, and players 2 and 3 prefer the outcome $x_{3}$ to $x_{1}$, so the players are involved in a decision problem that gives rise to the Condorcet paradox. A naïve approach would lead to the claim that majority voting over the alternatives results in a cycle.

Here we model majority voting over the alternatives by an explicit extensive form game. We assume that in each period $t$ some player, say player $i$, is selected randomly according to an a priori specified probability distribution. Player $i$ then decides either to make a proposal to the other two players, i.e. he proposes some outcome $x_{j}$, or he decides not to make a proposal, and the players reach period $t+1$. In the latter case, we will say that player $i$ makes proposal $x_{0}$. In the former case, the other two players vote sequentially. ${ }^{3}$

\footnotetext{
${ }^{3}$ Simultaneous voting may lead to undesirable equilibria due to coordination failures. For instance, the case where all players vote in favor of all proposals leads to an equilibrium, as there is no player who can gain by deviating.
} 
To avoid inessential multiplicity of equilibria, we assume that the player who ranks the outcome highest, is the first one to vote. ${ }^{4}$

Table 1 illustrates the order in which players vote given a proposal by some player. For instance, if player 1 proposes $x_{1}$, then we assume that first player 3 votes and next, conditional on a vote against by player 3 , player 2 . After player $i$ makes a proposal $x_{j}$, the first player to respond is denoted by $f_{j i}$, the second by $s_{j i}$.

\begin{tabular}{|c|c||c|c||c|c|}
\hline Proposal & Sequence & Proposal & Sequence & Proposal & Sequence \\
\hline$\left(x_{1}, 1\right)$ & $(3,2)$ & $\left(x_{1}, 2\right)$ & $(1,3)$ & $\left(x_{1}, 3\right)$ & $(1,2)$ \\
$\left(x_{2}, 1\right)$ & $(2,3)$ & $\left(x_{2}, 2\right)$ & $(1,3)$ & $\left(x_{2}, 3\right)$ & $(2,1)$ \\
$\left(x_{3}, 1\right)$ & $(3,2)$ & $\left(x_{3}, 2\right)$ & $(3,1)$ & $\left(x_{3}, 3\right)$ & $(2,1)$ \\
\hline
\end{tabular}

Table 1: The order of voting.

A voter casts a vote either in favor or against $x_{j}$. If the first voter casts a vote in favor of $x_{j}$, then the outcome $x_{j}$ is accepted and the game ends. If the first voter votes against $x_{j}$, then the second voter is allowed to vote. If the second voter casts a vote in favor of $x_{j}$, then the outcome $x_{j}$ is accepted and the game ends. Otherwise, period $t+1$ is reached. In period $t+1$ a new proposer is selected, and the entire procedure is repeated.

We assume that the probability of being recognized as a proposer is in each period $t$ given by $\rho=\left(\rho_{1}, \rho_{2}, \rho_{3}\right)$, where $\rho_{i}>0$ is the probability that player $i$ is recognized. The preferences of the players are represented by von-Neumann Morgenstern utility functions. We normalize utilities in such a way that the utility of disagreement forever is 0 for all players. Player $i$ 's utility of acceptance of proposal $x_{j}$ in period $t$ is equal to $u^{i}\left(x_{j}\right)$, so we consider the case without discounting. ${ }^{5}$ To satisfy $(2.1)$, we have that

$$
\begin{aligned}
& u^{1}\left(x_{1}\right)>u^{1}\left(x_{2}\right)>u^{1}\left(x_{3}\right) \geq 0, \\
& u^{2}\left(x_{2}\right)>u^{2}\left(x_{3}\right)>u^{2}\left(x_{1}\right) \geq 0, \\
& u^{3}\left(x_{3}\right)>u^{3}\left(x_{1}\right)>u^{3}\left(x_{2}\right) \geq 0 .
\end{aligned}
$$

For $i=1,2,3$, and $j=0,1,2,3$, we define $u_{j}^{i}=u^{i}\left(x_{j}\right), u_{j}=\left(u_{j}^{1}, u_{j}^{2}, u_{j}^{3}\right), u^{i}=\left(u_{0}^{i}, u_{1}^{i}, u_{2}^{i}, u_{3}^{i}\right)^{\top}$, and $u=\left(u^{1}, u^{2}, u^{3}\right)$. For $i=1,2,3$, we define $b_{i}, m_{i}$, and $w_{i}$ as the number of the alternative related to the best, middle, and worst outcome for player $i$. For instance, we have $b_{1}=1$, $m_{2}=3$, and $w_{3}=2$.

The sequence of proposers, proposals, and votes defines a history. A pure strategy of a player assigns an action to each history where he has to make a decision. We analyze mixed strategies that are defined in the usual way. Every mixed strategy implies a probability distribution $\left(\pi_{0}, \pi_{1}, \pi_{2}, \pi_{3}\right)$ over the four possible final outcomes, perpetual disagreement,

\footnotetext{
${ }^{4}$ Suppose player 1 proposes $x_{2}$, the best outcome for player 2 , and suppose that player 3 votes before player 2. The outcome $x_{2}$ is the worst outcome for player 3. Player 3 may nevertheless decide to vote in favor of $x_{2}$ since he knows that the proposal will be accepted anyhow by player 2 next and is therefore indifferent as far as his voting behavior is concerned.

${ }^{5}$ In the presence of discounting, equilibrium existence follows from standard results on equilibrium existence in stochastic games, see Fink (1964), Takahashi (1964), and Sobel (1971). For this class of games, Haller and Lagunoff (2000) show that the set of equilibria is generically finite. Herings and Peeters (2004) show that generically there is an odd number of equilibria and they provide an algorithm to compute the equilibrium that would be selected by a generalization of the tracing procedure.
} 
agreement on $x_{1}$, agreement on $x_{2}$, and agreement on $x_{3}$. So, any mixed strategy implies expected payoffs that are a weighted average of $u_{j}, j=0,1,2,3$, with weights $\pi_{j}$. Note that $\pi_{0}>0$ implies a positive probability of the players' worst possible outcome of perpetual disagreement. By (2.2)-(2.4), the expected payoffs are Pareto efficient if and only if $\pi_{1}+$ $\pi_{2}+\pi_{3}=1$. Pareto efficiency rules out perpetual disagreement, i.e. $\pi_{0}=0$.

Utility functions $u$ and recognition probabilities $\rho$ satisfying $(2.2)-(2.4)$ determine a game $G=(u, \rho)$ in extensive form. The class of all such games is denoted $\mathcal{G}$.

\section{Consistent Subgame Perfect Equilibria}

We analyze the game by examining its consistent subgame perfect equilibria. Suppose a player has to take an action at two subgames that are isomorphic in the sense that the corresponding preferences and action spaces are equivalent. Then consistency requires that the player take the same probability mix over actions in both subgames. This notion of consistency corresponds to the "invariance with respect to isomorphisms" of Harsanyi and Selten (1988), p. 73. A subgame perfect equilibrium in consistent strategies is called a consistent subgame perfect equilibrium (CPE).

Since the continuation game following the selection of a proposer is history independent, we can therefore restrict ourselves to strategies where the proposal is history independent. We denote by $p_{j}^{i}$ the probability that player $i$ proposes $x_{j}$ when he is recognized as proposer. Since the continuation game following a proposal by some player depends only on the proposal made and the identity of the proposer, the rejection probability may only depend on the identity of the proposer and the proposal made, but not on any other aspect of the history. The continuation game starting with the last responder to a proposal depends on the proposal made, but does not depend on the identity of the proposer. We therefore require the response of the last responder to be independent of the identity of the proposer.

The probability that player $i$ votes against a proposal $x_{j}$ by player $h$ is denoted by $r_{j h}^{i}$. As explained in the previous paragraph, the notion of a consistent strategy imposes the requirement $r_{32}^{1}=r_{33}^{1}, r_{11}^{2}=r_{13}^{2}$, and $r_{21}^{3}=r_{22}^{3}$. For notational simplicity, we define $r_{0 h}^{i}=1$. We define the set $P$ of admissible proposals by $P=P^{1} \times P^{2} \times P^{3}$, where

$$
P^{i}=\left\{p^{i} \in \mathbb{R}_{+}^{4} \mid \sum_{j=0,1,2,3} p_{j}^{i}=1\right\}, \quad i=1,2,3,
$$

and the set $R$ of admissible rejection probabilities by $R=R^{1} \times R^{2} \times R^{3}$, where

$$
R^{i}=\left\{r^{i} \in[0,1]^{4 \times 2} \mid \text { for } h, h^{\prime} \neq i, r_{0 h}^{i}=1 \text { and } r_{w_{i} h}^{i}=r_{w_{i} h^{\prime}}^{i}\right\} .
$$

Given consistent strategies, we can compute the expected utilities of the players. It will be useful to do so conditional on the identity of the proposer. The expected utility of player $i$ conditional on the proposer being player $h$ is denoted by $v_{h}^{i}$. Unconditional expected utility of player $i$ is $z^{i}$ and satisfies $z^{i}=\sum_{h=1}^{3} \rho_{h} v_{h}^{i}$.

Consistency of the strategies implies that the following recursive system holds,

$$
\begin{aligned}
v_{h}^{i} & =\sum_{j=0}^{3} p_{j}^{h}\left(1-r_{j h}^{h-1} r_{j h}^{h+1}\right) u_{j}^{i}+\sum_{j=0}^{3} p_{j}^{h} r_{j h}^{h-1} r_{j h}^{h+1} z^{i}, \quad i=1,2,3, h=1,2,3, \\
z^{i} & =\sum_{h=1}^{3} \rho_{h} v_{h}^{i}, \quad i=1,2,3 .
\end{aligned}
$$


In the definition of rejection probabilities above, we identify player 0 with player 3 , and player 4 with player 1. Equation (3.1) expresses that the expected utility of player $i$ conditional on the proposer being player $h$ is equal to the sum over all proposals of the probability that player $h$ makes this proposal and that it is accepted by the other players times the utility of the proposal plus the probability that player $h$ makes a proposal that is rejected times the utility $z^{i}$.

Let $(p, r)$ be a CPE. No player has a profitable deviation, so in particular, no player has a profitable one-shot deviation. The absence of a profitable one-shot deviation is equivalent to the following set of implications, where in (3.3) it holds that $i \in\{1,2,3\}$ and $j \in\{0,1,2,3\}$,

$$
\begin{aligned}
p_{j}^{i}>0 & \Rightarrow\left(1-r_{j i}^{i-1} r_{j i}^{i+1}\right) u_{j}^{i}+r_{j i}^{i-1} r_{j i}^{i+1} z^{i}=\max _{k \in\{0,1,2,3\}}\left(1-r_{k i}^{i-1} r_{k i}^{i+1}\right) u_{k}^{i}+r_{k i}^{i-1} r_{k i}^{i+1} z^{i} \\
r_{j h}^{i}>0 & \Rightarrow z^{i} \geq u_{j}^{i} \text { or } r_{j h}^{i^{\prime}}=0, \quad j=1,2,3, h=1,2,3, i=f_{j h}, i^{\prime}=s_{j h} \\
r_{j h}^{i}<1 & \Rightarrow z^{i} \leq u_{j}^{i} \text { or } r_{j h}^{i^{\prime}}=0, \quad j=1,2,3, h=1,2,3, i=f_{j h}, i^{\prime}=s_{j h} \\
r_{j h}^{i}>0 & \Rightarrow z^{i} \geq u_{j}^{i}, \quad j=1,2,3, h=1,2,3, i=s_{j h} \\
r_{j h}^{i}<1 & \Rightarrow z^{i} \leq u_{j}^{i}, \quad j=1,2,3, h=1,2,3, i=s_{j h}
\end{aligned}
$$

Equality (3.3) expresses that a proposal that is made with positive probability maximizes the sum of instantaneous utility and continuation utility. We obtain (3.4) by observing that $r_{j h}^{i}>0$ implies $\left(1-r_{j h}^{i^{\prime}}\right) u_{j}^{i}+r_{j h}^{i^{\prime}} z^{i} \geq u_{j}^{i}$; the utility to player $i$ of accepting proposal $j$ by player $h$ should weakly exceed the utility of rejection. This inequality is equivalent to $z^{i} \geq u_{j}^{i}$ or $r_{j h}^{i^{\prime}}=0$. The derivation of (3.5)-(3.7) is analogous. Observe that (3.4)-(3.5) correspond to the cases where player $i$ is the first voter to accept or reject a proposal, and (3.6)-(3.7) to the cases where player $i$ is the second voter to make such a decision.

In the following, let $(p, r)$ be a given CPE, inducing continuation utilities $v$ and $z$. We will analyze how $(p, r)$ depends on the primitives of the model, $u^{i}$ and $\rho_{i}, i=1,2,3$. We continue by deriving several properties regarding the structure of CPEs and conclude this section with two theorems. All these results reduce (3.3)-(3.7) to a considerably simpler system that is analyzed in the next section. These properties are derived step by step.

The first property states that forever delay with probability 1 is not an equilibrium. Indeed, forever delay with probability 1 implies, for every $i, z^{i}=0$ and $v^{i}=0$. By (3.3), player 1 should obtain expected utility 0 from proposing $x_{1}$, which can only be the case if $r_{11}^{2}=r_{11}^{3}=1$. By (3.4), $r_{11}^{3}=1$ implies $z^{3} \geq u_{1}^{3}$ or $r_{11}^{2}=0$. This leads to a contradiction as $z^{3}=0<u_{1}^{3}$ and $r_{11}^{2}=1$. It follows that forever delay with probability 1 is not an equilibrium.

Using the conclusion of the previous paragraph, we have that some player makes with positive probability a proposal that is accepted with positive probability. Since such a player is recognized with strictly positive probability, the probability that negotiations have not terminated at period $t$ goes to zero as $t$ goes to infinity. In other words, at any equilibrium there is an agreement in finite expected time with probability 1 . So, $\pi_{0}=0$ and each $z^{i}$ is therefore a weighted average of $u_{j}^{i}, j=1,2,3$, with $\pi_{j} \in[0,1]$ such that $\pi_{1}+\pi_{2}+\pi_{3}=1$ independent of $i$. It holds in particular that $\pi_{j}>0$ for some $j=1,2,3$ and $\left(z^{1}, z^{2}, z^{3}\right) \neq 0$. By Herings and Houba (2010), it follows that (3.1)-(3.7) together with $\left(z^{1}, z^{2}, z^{3}\right) \neq 0$ is necessary and sufficient for $(p, r) \in P \times R$ to be a CPE inducing continuation utilities $v$ and $z$. As a corollary, some agreement with probability one implies 
the following result.

\section{TheOREM 3.1 Every CPE is Pareto efficient.}

This result shows that bargaining under exogenous recognition probabilities is a road map to overcome the Condorcet paradox. The generality of this result holds for all parameter values for which the bargaining model has at least one $\mathrm{CPE}$ and it is therefore unrestricted in the sense that the necessary and sufficient conditions for the existence of a CPE immediately imply Pareto efficiency. Given the indeterminacy of many cooperative theories about the Condorcet paradox, this result already suggests a great potential in further elaborating the bargaining approach.

In the next step, we show that each player $i$ has $z^{i}$ strictly exceeding the utility $u_{w_{i}}^{i}$ of his worst outcome. Suppose, on the contrary, that player $i$ has $z^{i}=u_{w_{i}}^{i}$. The probability is therefore 1 that the outcome $x_{w_{i}}$ is accepted at some point in time, since otherwise the utility of $i$ strictly exceeds $u_{w_{i}}^{i}$. Therefore, with probability 1 , player $i-1$ gets utility $u_{b_{i-1}}^{i-1}$ and player $i+1$ utility $u_{m_{i+1}}^{i+1}$. Moreover, it follows that $z^{i-1}=u_{b_{i-1}}^{i-1}$ and $z^{i+1}=u_{m_{i+1}}^{i+1}$. Since $u_{m_{i}}^{i-1}=u_{w_{i-1}}^{i-1}<u_{b_{i-1}}^{i-1}=z^{i-1}$, (3.7) yields that player $i-1$ rejects proposal $x_{m_{i}}$ by player $i$ with probability 1 , so $r_{m_{i} i}^{i-1}=1$. Since $r_{m_{i} i}^{i-1}=1$ and $z^{i+1}=u_{m_{i+1}}^{i+1}<u_{b_{i+1}}^{i+1}=u_{m_{i}}^{i+1}$, (3.4) yields that player $i+1$ rejects proposal $x_{m_{i}}$ by player $i$ with probability 0 . Proposal $x_{m_{i}}$ by player $i$ is therefore accepted with probability 1 , so $v_{i}^{i} \geq u_{m_{i}}^{i}>u_{w_{i}}^{i}$. Since $v_{i-1}^{i} \geq u_{w_{i}}^{i}$ and $v_{i+1}^{i} \geq u_{w_{i}}^{i}$, we find that $u_{w_{i}}^{i}=z^{i}=\rho_{1} v_{1}^{i}+\rho_{2} v_{2}^{i}+\rho_{3} v_{3}^{i}>u_{w_{i}}^{i}$, a contradiction. We conclude that each player has $z^{i}$ strictly exceeding $u_{w_{i}}^{i}$, i.e.

$$
z^{i}>u_{w_{i}}^{i}, \quad i=1,2,3 .
$$

We show next that each player $i$ has $z^{i}$ strictly lower than the utility of his best outcome, $u_{b_{i}}^{i}$. If some player $i$ has $z^{i}=u_{b_{i}}^{i}$, then outcome $x_{b_{i}}$ is accepted with probability 1 , so $z^{i+1}=u_{b_{i}}^{i+1}=u_{w_{i+1}}^{i+1}$, a contradiction to (3.8). We have found that

$$
z^{i}<u_{b_{i}}^{i}, \quad i=1,2,3 .
$$

Next, any voter rejects his worst alternative for sure. To see this, when player $h$ proposes outcome $x_{w_{i}}, i \neq h$, then player $i$ is the last one to vote. It holds by (3.8) that $z^{i}>u_{w_{i}}^{i}$, so by $(3.7), r_{w_{i} h}^{i}=1$. We have shown that

$$
r_{w_{i} h}^{i}=1, \quad i=1,2,3, h \neq i .
$$

Notice that (3.10) implies proposer-independent probabilities of rejecting $r_{32}^{1}=r_{33}^{1}, r_{11}^{2}=$ $r_{13}^{2}$, and $r_{21}^{3}=r_{22}^{3}$ as required in the definition of $R$.

We continue by establishing that, independent of who proposes, the recognized player can always conclude the bargaining for sure by proposing either his worst or his middle alternative. Consider a proposal $x_{m_{i}}$ by player $i$, so player $i$ proposes his middle outcome and player $i+1$, for whom it is the best alternative, votes before player $i-1$. We argue that this proposal will be accepted with probability 1 by player $i+1$, i.e. $r_{m_{i} i}^{i+1}=0$. By (3.10), since $m_{i}=w_{i-1}, r_{m_{i} i}^{i-1}=1$. Using that $m_{i}=b_{i+1}$, we know by $(3.9), u_{m_{i}}^{i+1}>z^{i+1}$. Since $r_{m_{i} i}^{i-1}=1$, we use (3.4) to conclude that $r_{m_{i} i}^{i+1}=0$. We have derived that

$$
r_{21}^{2}=r_{32}^{3}=r_{13}^{1}=0 \text {. }
$$


Consider now a proposal $x_{w_{i}}$ by player $i$ meaning player $i$ proposes his worst outcome. We argue that this proposal will be accepted with probability one, i.e. $r_{w_{i} i}^{i-1} r_{w_{i} i}^{i+1}=0$. Since $w_{i}=b_{i-1}$, it follows from (3.9) that $u_{w_{i}}^{i-1}>z^{i-1}$, so by (3.4), $r_{w_{i} i}^{i-1}=0$ or $r_{w_{i} i}^{i+1}=0$. We have derived that

$$
r_{31}^{2} r_{31}^{3}=r_{12}^{1} r_{12}^{3}=r_{23}^{1} r_{23}^{2}=0 .
$$

As a corollary, a recognized player will never propose his worst alternative, because he can do strictly better by proposing his second best, i.e.

$$
p_{w_{i}}^{i}=0, \quad i=1,2,3 .
$$

We have already argued that each $z^{i}$ is a weighted average of $u_{j}^{i}, j=1,2,3$, with weights $\pi_{j}$ independent of $i$. We argue next that all weights are strictly positive. If only one weight would be strictly positive, we would get a contradiction to (3.8) for some $i$. Suppose that exactly two weights are strictly positive, without loss of generality the weights $\pi_{1}$ on outcome $x_{1}$ and $\pi_{2}$ on $x_{2}$ sum up to one and $\pi_{3}=0$, so $z^{i}=\pi_{1} u_{1}^{i}+\pi_{2} u_{2}^{i}$. For the equality $\pi_{3}=0$ to hold, the proposal $x_{3}$ by player 3 should be rejected with probability 1 . The proposal $x_{1}$ by player 3 is accepted with probability 1 according to (3.11). We can now use (3.3) to conclude that $p_{0}^{3}=p_{3}^{3}=0$, and since $p_{2}^{3}=0$ by (3.13), we know that $p_{1}^{3}=1$. From (3.4) and (3.10), the proposal $x_{1}$ by player 1 is accepted with probability 1 by player 3 . The proposal $x_{2}$ by player 1 is accepted with probability 1 according to (3.11). We can now use (3.3) to conclude that $p_{0}^{1}=p_{2}^{1}=0$, and since $p_{3}^{1}=0$ by (3.13), we know that $p_{1}^{1}=1$. A proposal $x_{2}$ by player 2 would be rejected with probability 1 by player 1 using (3.5) and the fact that $r_{22}^{3}=1$ by (3.10). It now follows that $\pi_{1}=1$, a contradiction to (3.8). We conclude that all weights are strictly positive,

$$
\pi_{1}, \pi_{2}, \pi_{3}>0
$$

So far we have characterized the set of consistent subgame perfect equilibria as the set of solutions $(p, r) \in P \times R$ to (3.1)-(3.7) leading to utility levels $z \neq 0$. The next result claims that there is no loss of generality in restricting the analysis to proposer-independent rejection probabilities.

THEOREM 3.2 If $(p, r) \in P \times R$ is a consistent subgame perfect equilibrium inducing utilities $v$ and $z$, then there is also a consistent subgame perfect equilibrium $(p, \bar{r}) \in P \times R$ inducing utilities $v$ and $z$ such that $\bar{r}$ is proposer-independent, i.e. $\bar{r}_{j h}^{i}=\bar{r}_{j h^{\prime}}^{i}$ for all $i, j, h$, and $h^{\prime}$. Moreover, $\bar{r}$ can be defined by setting, for $i=1,2,3, \bar{r}_{b_{i} i+1}^{i}=0, \bar{r}_{m_{i} i-1}^{i}=r_{m_{i} i+1}^{i}$, and $\bar{r}_{j h}^{i}=r_{j h}^{i}$, otherwise.

Proof: Assume that $(p, r) \in P \times R$ satisfies (3.1)-(3.7). We show that $(p, \bar{r})$ satisfies (3.1)-(3.7), where $\bar{r}$ is as defined in Theorem 3.1. We verify first that $\bar{r}$ is proposer independent. Indeed, for $i=1,2,3$, we have the following. It holds by definition that $\bar{r}_{0 h}^{i}=1$, $h \neq i$. We have by definition that $\bar{r}_{b_{i} i+1}^{i}=0$ and $\bar{r}_{b_{i} i-1}^{i}=r_{b_{i} i-1}^{i}=0$, where the last equality holds by (3.11). Also it holds by definition that $\bar{r}_{m_{i} i+1}^{i}=r_{m_{i} i+1}^{i}$ and $\bar{r}_{m_{i} i-1}^{i}=r_{m_{i} i+1}^{i}$, so $\bar{r}_{m_{i} i+1}^{i}=\bar{r}_{m_{i} i-1}^{i}$. Finally, we have $r_{w_{i} h}^{i}=1, h \neq i$, by $(3.10)$, and $\bar{r}_{w_{i} h}^{i}=r_{w_{i} h}^{i}, h \neq i$, by definition.

We show next that

$$
r_{j h}^{h-1} r_{j h}^{h+1}=\bar{r}_{j h}^{h-1} \bar{r}_{j h}^{h+1}, \quad h=1,2,3, j=0,1,2,3 .
$$


For $j=0$, this follows immediately from the definition of $R$. Three possible cases remain: (i) $j=b_{h}$, (ii) $j=m_{h}$, and (iii) $j=w_{h}$.

Case (i), $j=b_{h}$. Since $b_{h}=m_{h-1}=w_{h+1}$, we have

$$
\bar{r}_{b_{h} h}^{h-1} \bar{r}_{b_{h} h}^{h+1}=\bar{r}_{m_{h-1} h}^{h-1} \bar{r}_{w_{h+1} h}^{h+1}=r_{m_{h-1} h}^{h-1} r_{w_{h+1} h}^{h+1}=r_{b_{h} h}^{h-1} r_{b_{h} h}^{h+1},
$$

where the second equality follows by definition of $\bar{r}$.

Case (ii), $j=m_{h}$. Since $m_{h}=w_{h-1}=b_{h+1}$, we obtain

$$
\bar{r}_{m_{h} h}^{h-1} \bar{r}_{m_{h} h}^{h+1}=\bar{r}_{w_{h-1} h}^{h-1} \bar{r}_{b_{h+1} h}^{h+1}=r_{w_{h-1} h}^{h-1} r_{b_{h+1} h}^{h+1}=r_{m_{h} h}^{h-1} r_{m_{h} h}^{h+1},
$$

where the second equality follows by definition of $\bar{r}$.

Case (iii), $j=w_{h}$. By (3.12), it holds that $r_{w_{h} h}^{h-1} r_{w_{h} h}^{h+1}=0$. Since $w_{h}=b_{h-1}$, we have that $\bar{r}_{w_{h} h}^{h-1}=\bar{r}_{b_{h-1} h}^{h-1}=0$, where the last equality follows by definition of $\bar{r}$. It follows that $\bar{r}_{w_{h} h}^{h-1} \bar{r}_{w_{h} h}^{h+1}=0$.

Using (3.14) we have that $(p, \bar{r}, v, z)$ satisfies (3.1), (3.2), and (3.3). We verify next that $(p, \bar{r}, v, z)$ satisfies (3.4)-(3.7). Consider some $\bar{r}_{j h}^{i}$. If $j=b_{i}$, then $\bar{r}_{j h}^{i}=0$ and $i=f_{j h}$, so (3.4), (3.6), and (3.7) hold trivially. Implication (3.5) holds as well, since $z^{i}<u_{b_{i}}^{i}$ by (3.9). If $j=w_{i}$, then $\bar{r}_{j h}^{i}=r_{j h}^{i}=1$ by (3.10), so (3.5) and (3.7) hold trivially. Since by (3.8) $z^{i}>u_{w_{i}}^{i}$, we find that (3.4) and (3.6) also hold. If $j=m_{i}$ and $h=i+1$, then $i=f_{j h}$, so (3.6) and (3.7) hold trivially. We have that $\bar{r}_{j h}^{i}=r_{j h}^{i}$ and $\bar{r}_{j h}^{i^{\prime}}=\bar{r}_{w_{i-1} i+1}^{i-1}=r_{w_{i-1} i+1}^{i-1}=r_{j h}^{i^{\prime}}$, so (3.4) and (3.5) hold. Finally, we consider the case where $j=m_{i}$ and $h=i-1$, so $i=s_{j h}$ and (3.4) and (3.5) hold trivially. Assume $\bar{r}_{j h}^{i}>0$. Since by definition $\bar{r}_{j h}^{i}=\bar{r}_{m_{i} i-1}^{i}=r_{m_{i} i+1}^{i}$, we have $r_{m_{i} i+1}^{i}>0$, so by (3.4) $z^{i} \geq u_{m_{i}}^{i}$ or $r_{m_{i} i+1}^{i-1}=0$. Since $m_{i}=w_{i-1}$, (3.10) implies $r_{m_{i} i+1}^{i-1}=1$, so $z^{i} \geq u_{m_{i}}^{i}$. It follows that (3.6) holds. Assume $\bar{r}_{j h}^{i}<1$. Since by definition $\bar{r}_{j h}^{i}=\bar{r}_{m_{i} i-1}^{i}=r_{m_{i} i+1}^{i}$, we have $r_{m_{i} i+1}^{i}<1$, so by $(3.5) z^{i} \leq u_{m_{i}}^{i}$ or $r_{m_{i} i+1}^{i-1}=0$. Since $m_{i}=w_{i-1}$, (3.10) implies $r_{m_{i} i+1}^{i-1}=1$, so $z^{i} \geq u_{m_{i}}^{i}$. It follows that (3.7) holds. Q.E.D.

The bargaining model may admit multiple CPEs that support the same utilities $z$ and $v$. Such multiplicity is inessential in terms of characterizing expected equilibrium utilities. The importance of Theorem 3.2 is that it identifies a systematic way in reducing the number of unknowns without discarding any pair $z$ and $v$ of equilibrium utilities. This theorem implies that every pair $z$ and $v$ of CPE utilities can be supported by proposer-independent rejection probabilities and analyzing such probabilities reduces the number of unknowns.

Since some of the previous results, such as each player always rejects his worst alternative, already hinted at proposer-independent probabilities, Theorem 3.2 is especially selective in subgames in which the current proposer chose to put up his worst alternative for a vote. Recall that such subgames are never reached in any CPE, but the CPE implies that such a proposal would be accepted with probability one, i.e. player $i-1$ proposed $w_{i-1}$ and $r_{b_{i+1} i-1}^{i+1} r_{m_{i} i-1}^{i}=0$. Of course, $r_{m_{i} i-1}^{i}$ has to satisfy the equilibrium condition in the subgame where $i+1$, for whom it is the best alternative, voted against. In case $r_{m_{i} i-1}^{i}=0$ would be the equilibrium probability, the requirement $r_{b_{i+1} i-1}^{i+1} r_{m_{i} i-1}^{i}=0$ leads to indeterminacy of $r_{b_{i+1} i-1}^{i+1} \in[0,1]$. So, the CPE strategies do not force a player to accept for sure his best alternative in this case, in case the next voter will accept for sure. ${ }^{6}$ More generally, in case

\footnotetext{
${ }^{6}$ Since a player always rejects his third-best alternative, this indeterminacy is ruled out when player $i$ proposed $w_{i-1}$ instead of player $i-1$, because then $i+1$ votes before $i-1$, who rejects for sure, making $i+1$ accept for sure.
} 
player $i$ would be indifferent between accepting $m_{i}$ proposed by player $i-1$ or rejecting $m_{i}$, then $r_{b_{i+1} i-1}^{i+1} r_{m_{i} i-1}^{i}=0$ implies indeterminacy of either $r_{b_{i+1} i-1}^{i+1} \in[0,1]$ or $r_{m_{i} i-1}^{i} \in[0,1]$. In that case, Theorem 3.2 identifies $\bar{r}_{b_{i+1} i-1}^{i+1}=\bar{r}_{b_{i+1} i}^{i+1}=0$ and $\bar{r}_{m_{i} i-1}^{i}=\bar{r}_{m_{i} i+1}^{i}=r_{m_{i} i+1}^{i}$. Apart from being simpler, the proposer-independent rejection probabilities identified in Theorem 3.2 also are more intuitive. In particular, other CPE strategies supporting the same $z$ and $v$ are less intuitive when refraining from accepting a best alternative for sure.

By virtue of Theorem 3.2, we may drop the subscript indicating the proposer from the notation of a rejection probability. The set of proposer-independent rejection probabilities is $Q=Q^{1} \times Q^{2} \times Q^{3}$, where

$$
Q^{i}=\left\{q^{i} \in[0,1]^{4} \mid q_{0}^{i}=1\right\} .
$$

We have already argued that each $z^{i}$ is a weighted average of $u_{j}^{i}, j=1,2,3$, with weights $\pi_{j}$ independent of $i$. We argue next that all these weights are strictly positive. If only one weight would be strictly positive, we would get a contradiction to (3.8) for some $i$. Suppose that exactly two weights are strictly positive, without loss of generality the weights $\pi_{1}$ on outcome $x_{1}$ and $\pi_{2}$ on $x_{2}$ sum up to one and $\pi_{3}=0$, so $z^{i}=\pi_{1} u_{1}^{i}+\pi_{2} u_{2}^{i}$. For the equality $\pi_{3}=0$ to hold, the proposal $x_{3}$ by player 3 should be rejected with probability 1 . The proposal $x_{1}$ by player 3 is accepted with probability 1 according to (3.11). We can now use (3.3) to conclude that $p_{0}^{3}=p_{3}^{3}=0$, and since $p_{2}^{3}=0$ by (3.13), we know that $p_{1}^{3}=1$. From (3.4) and (3.10), the proposal $x_{1}$ by player 1 is accepted with probability 1 by player 3. The proposal $x_{2}$ by player 1 is accepted with probability 1 according to (3.11). We can now use (3.3) to conclude that $p_{0}^{1}=p_{2}^{1}=0$, and since $p_{3}^{1}=0$ by $(3.13)$, we know that $p_{1}^{1}=1$. A proposal $x_{2}$ by player 2 would be rejected with probability 1 by player 1 using (3.5) and the fact that $r_{22}^{3}=1$ by (3.10). It now follows that $\pi_{1}=1$, a contradiction to (3.8). We conclude that all weights are strictly positive,

$$
\pi_{1}, \pi_{2}, \pi_{3}>0
$$

Recall that at any equilibrium there is agreement within finite expected time with probability 1 . Consequently, also $\pi_{1}+\pi_{2}+\pi_{3}=1$.

We summarize what we have found so far. At a $\operatorname{CPE}(p, \bar{q}) \in P \times Q$ an agreement is eventually reached with probability one, and all outcomes occur with strictly positive probability. Therefore, any CPE is Pareto efficient. The expected equilibrium utility is strictly in between the utility of a player's worst outcome and a player's best outcome. If a player proposes his middle outcome, it is accepted for sure since for every player $i$ it holds that $\bar{q}_{b_{i}}^{i}=0$. If a player would propose his worst outcome, it would also be accepted for sure, implying that no player ever proposes his worst outcome, i.e. for every player $i$, $p_{w_{i}}^{i}=0$. Moreover, a player rejects his worst outcome for sure, i.e. for every $i, \bar{q}_{w_{i}}^{i}=1$. The only variables that have not yet been determined are $p_{0}^{i}, p_{m_{i}}^{i}, p_{b_{i}}^{i}$, and $\bar{q}_{m_{i}}^{i}$.

It seems intuitive that in any $\operatorname{CPE}(p, \bar{q}) \in P \times Q$ with utilities $v$ and $z$, the recognized player is better off making some proposal instead of not making a proposal, i.e. $p_{0}^{i}=0$. As we will show in Section 4.1, for some parameter values we can have $p_{0}^{i}>0$ for some $i$. In such cases, however, we will show that there also exists a closely related $\operatorname{CPE}(\bar{p}, \bar{q}) \in P \times Q$ with $\bar{p}_{0}^{i}=0$ for all players $i$ that yields the same pair of utilities $v$ and $z$. This implies that in characterizing the set of CPEs, we may first search for equilibria $(\bar{p}, \bar{q}) \in P \times Q$ with $\bar{p}_{0}^{i}=0$ for all players $i$. This is without loss of generality in the sense that if there is 
an equilibrium $(p, \bar{q})$ involving $p_{0}^{i} \neq 0$ for some $i$, then there is also an equilibrium $(\bar{p}, \bar{q})$ where $\bar{p}_{b_{i}}^{i}=p_{b_{i}}^{i}+p_{0}^{i}, \bar{p}_{0}^{i}=0$, and $\bar{p}_{j}=p_{j}$ for $j \neq 0, b_{i}$. Indeed, by the definition of equilibrium it should not be profitable to propose $x_{b_{i}}$ instead of $x_{0}$. This means that either $x_{b_{i}}$ is rejected with probability 1 when proposed or $z^{i}=u_{b_{i}}^{i}$. The latter case contradicts (3.9), so we only have to consider the former case. Since we are considering CPEs, the change in strategy from not making a proposal to proposing one's best outcome, which is rejected with probability 1 , is not affecting the payoffs of anyone, and is also an equilibrium. The next proposition gives an easy characterization of CPEs $(\bar{p}, \bar{q}) \in P \times Q$ where no player gives up the right to make a proposal, i.e. $\bar{p}_{0}^{i}=0$ for all players $i$.

THEOREM 3.3 The strategy profile $(\bar{p}, \bar{q}) \in P \times Q$ is a CPE where all players make a proposal with probability one if and only if for $i=1,2,3, \bar{p}_{0}^{i}=\bar{p}_{w_{i}}^{i}=0, \bar{q}_{b_{i}}^{i}=0, \bar{q}_{w_{i}}^{i}=1$, and there is $\bar{\pi} \in \mathbb{R}_{++}^{3}$ and $\bar{z} \in \mathbb{R}^{3}$ such that

$$
\begin{aligned}
& \bar{p}_{m_{i}}^{i}>0 \Rightarrow u_{m_{i}}^{i} \geq\left(1-\bar{q}_{m_{i-1}}^{i-1}\right) u_{b_{i}}^{i}+\bar{q}_{m_{i-1}}^{i-1} \bar{z}^{i}, \quad i=1,2,3, \\
& \bar{p}_{b_{i}}^{i}>0 \Rightarrow\left(1-\bar{q}_{m_{i-1}}^{i-1}\right) u_{b_{i}}^{i}+\bar{q}_{m_{i-1}}^{i-1} \bar{z}^{i} \geq u_{m_{i}}^{i}, \quad i=1,2,3, \\
& \bar{q}_{m_{i}}^{i}>0 \Rightarrow \bar{z}^{i} \geq u_{m_{i}}^{i}, \quad i=1,2,3, \\
& \bar{q}_{m_{i}}^{i}<1 \Rightarrow \bar{z}^{i} \leq u_{m_{i}}^{i}, \quad i=1,2,3, \\
& \bar{\pi}_{1} u_{1}^{i}+\bar{\pi}_{2} u_{2}^{i}+\bar{\pi}_{3} u_{3}^{i}=\bar{z}^{i}, \quad i=1,2,3, \\
& \bar{\pi}_{1}+\bar{\pi}_{2}+\bar{\pi}_{3}=1, \\
& \bar{\pi}_{1}: \bar{\pi}_{2}=\rho_{1} \bar{p}_{b_{1}}^{1}\left(1-\bar{q}_{1}^{3}\right)+\rho_{3} \bar{p}_{m_{3}}^{3}: \rho_{2} \bar{p}_{b_{2}}^{2}\left(1-\bar{q}_{2}^{1}\right)+\rho_{1} \bar{p}_{m_{1}}^{1}, \\
& \bar{\pi}_{2}: \bar{\pi}_{3}=\rho_{2} \bar{p}_{b_{2}}^{2}\left(1-\bar{q}_{2}^{1}\right)+\rho_{1} \bar{p}_{m_{1}}^{1}: \rho_{3} \bar{p}_{b_{3}}^{3}\left(1-\bar{q}_{3}^{2}\right)+\rho_{2} \bar{p}_{m_{2}}^{2} .
\end{aligned}
$$

\section{PROOF:}

$(\Rightarrow)$ This direction follows immediately from the results derived in this section.

$(\Leftarrow)$ This direction follows by defining, for $h=1,2,3, i=1,2,3$, and $j=0,1,2,3$,

$$
\begin{aligned}
\bar{v}_{h}^{i} & =\bar{p}_{m_{h}}^{h} u_{m_{h}}^{i}+\bar{p}_{b_{h}}^{h}\left(1-\bar{q}_{b_{h}}^{h-1}\right) u_{b_{h}}^{i}+\bar{p}_{b_{h}}^{h} \bar{q}_{b_{h}}^{h-1} \bar{z}^{i}, \\
\bar{r}_{j h}^{i} & =\bar{q}_{j}^{i},
\end{aligned}
$$

and verifying that a solution $(\bar{p}, \bar{q}, \bar{\pi}, \bar{z})$ to $(3.15)-(3.22)$ inducing expected utilities $\bar{v}$ and rejection probabilities $\bar{r}$ leads to a solution $(\bar{p}, \bar{r}, \bar{v}, \bar{z})$ to $(3.1)-(3.7)$ with $\bar{z} \neq 0$. Q.E.D.

\section{Equilibrium Types}

The results of the previous section show that player $i$ faces two dilemmas. First, by what probability will I propose my middle alternative $x_{m_{i}}$ knowing it will be accepted for sure instead of taking the risk involved in proposing my best alternative. Second, by what probability will I reject my middle alternative $x_{m_{i}}$ when offered to me knowing that rejecting it leads to a gamble over my top three alternatives including my worst. These dilemmas concern the equilibrium values of $p_{m_{i}}^{i}$ and $q_{m_{i}}^{i}$ that also pin down $p_{b_{i}}^{i}=1-p_{m_{i}}^{i}$.

The answer to the first dilemma results in four possible types of equilibrium. The first one is where all players $i$ have a strictly positive $\bar{p}_{m_{i}}^{i}$. The other types of equilibria are characterized by two, one, and none of the players having a strictly positive $\bar{p}_{m_{i}}^{i}$. We devote 
one subsection to each of these types. The answer to the second dilemma is intimately related to the value of $\bar{z}^{i}$. We will show that all equilibria have the property that $\bar{z}^{i} \leq u_{m_{i}}^{i}$. Then it follows that $\bar{q}_{m_{i}}^{i}=0$ if $\bar{z}^{i}<u_{m_{i}}^{i}$, whereas strictly positive values for $\bar{q}_{m_{i}}^{i}$ are admitted when $\bar{z}^{i}=u_{m_{i}}^{i}$.

For the cases with one and none of the players having a strictly positive $\bar{p}_{m_{i}}^{i}$ there exist two, respectively four, equilibrium subtypes, depending on the number of players with $\bar{z}^{i}=u_{m_{i}}^{i}$. For the other cases we do not have to distinguish subtypes. In the type of equilibrium where one player has a strictly positive $\bar{p}_{m_{i}}^{i}$, without loss of generality player 1 , we can show that in equilibrium $\bar{q}_{1}^{3}=0$ and $\bar{q}_{3}^{2}>0$. Two equilibrium subtypes occur for $\bar{z}^{1}=u_{2}^{1}$ and $\bar{z}^{1}<u_{2}^{1}$, respectively. In the equilibrium type where no player has a strictly positive $\bar{p}_{m_{i}}^{i}$, four subtypes occur, with one subtype for each possible number of players with $\bar{z}^{i}=u_{m_{i}}^{i}$. The equilibrium subtypes are analyzed in subsubsections. In the final subsection, we provide a table that summarizes the results obtained for all subtypes.

\subsection{Three Players with $\bar{p}_{m_{i}}^{i}>0$.}

In this subsection we are analyzing equilibria where every player makes a proposal with probability one, proposes his middle alternative with strictly positive probability, does not propose his worst alternative, and may propose his best alternative with strictly positive probability less than one.

Consider a game $(u, \rho) \in \mathcal{G}$ and let $\left(\left(\bar{p}_{b_{i}}^{i}, \bar{p}_{m_{i}}^{i}, \bar{q}_{m_{i}}^{i}, \bar{z}^{i}\right)_{i=1,2,3}, \bar{\pi}\right)$ be a solution to (3.15)(3.22) with $\bar{p}_{m_{i}}^{i}>0, i=1,2$, 3. From (3.15), for every $i, u_{m_{i}}^{i} \geq\left(1-\bar{q}_{m_{i-1}}^{i-1}\right) u_{b_{i}}^{i}+\bar{q}_{m_{i-1}}^{i-1} \bar{z}^{i}$, so $\bar{z}^{i} \leq u_{m_{i}}^{i}$. We argue next that for every $i, \bar{z}^{i}=u_{m_{i}}^{i}$. Suppose, for some $i, \bar{z}^{i}<u_{m_{i}}^{i}$. Then $\bar{q}_{m_{i}}^{i}=0$ by (3.17), so $\bar{p}_{m_{i+1}}^{i+1}=0$ by (3.15), a contradiction since we are considering the case $\bar{p}_{m_{i+1}}^{i+1}>0$. It follows that for every $i, \bar{z}^{i}=u_{m_{i}}^{i}$, and by $(3.15), \bar{q}_{m_{i-1}}^{i-1}=1$, so the proposal $x_{m_{i}}$ by a player $i$ is accepted with probability 1 and the proposal $x_{b_{i}}$ by a player $i$ is rejected with probability 1 . Note that since a proposal $x_{b_{i}}$ by player $i$ is rejected for sure, player $i$ is indifferent between making such a proposal and giving up the right to propose, i.e. propose $x_{0}$.

Equations (3.19)-(3.20) now reduce to the system

$$
\begin{aligned}
\bar{\pi}_{1} u_{1}^{1}+\bar{\pi}_{2} u_{2}^{1}+\bar{\pi}_{3} u_{3}^{1} & =u_{2}^{1}, \\
\bar{\pi}_{1} u_{1}^{2}+\bar{\pi}_{2} u_{2}^{2}+\bar{\pi}_{3} u_{3}^{2} & =u_{3}^{2}, \\
\bar{\pi}_{1} u_{1}^{3}+\bar{\pi}_{2} u_{2}^{3}+\bar{\pi}_{3} u_{3}^{3} & =u_{1}^{3}, \\
\bar{\pi}_{1}+\bar{\pi}_{2}+\bar{\pi}_{3} & =1 .
\end{aligned}
$$

Equations (3.21) and (3.22) can be simplified to

$$
\bar{\pi}_{1}: \bar{\pi}_{2}: \bar{\pi}_{3}=\rho_{3} \bar{p}_{m_{3}}^{3}: \rho_{1} \bar{p}_{m_{1}}^{1}: \rho_{2} \bar{p}_{m_{2}}^{2} .
$$

Whenever utilities are such that (4.1)-(4.4) has a solution $\bar{\pi} \gg 0$, an equilibrium of the type we are looking for in this subsection exists. We derive now under what assumptions on utilities such a solution $\bar{\pi}$ exists. We will show that there is at most one solution, so a solution, if it exists, is unique.

It will be useful to introduce the notation $\Delta_{k}^{i}$ for the utility difference between the $k$ best outcome and $(k+1)$-best outcome for agent $i$. More precisely, we define $\Delta_{1}^{i}=u_{b_{i}}^{i}-u_{m_{i}}^{i}$ and $\Delta_{2}^{i}=u_{m_{i}}^{i}-u_{w_{i}}^{i}$. Moreover, we define $\Delta_{3}^{i}=\Delta_{1}^{i}+\Delta_{2}^{i}$, so it holds that $\Delta_{3}^{i}=u_{b_{i}}^{i}-u_{w_{i}}^{i}$. 
From equalities (4.1) and (4.4), we obtain

$$
\left(1-\bar{\pi}_{2}\right) \Delta_{2}^{1}=\bar{\pi}_{1} \Delta_{3}^{1} .
$$

Combining (4.2) and (4.4) leads to

$$
\begin{aligned}
\bar{\pi}_{1} \Delta_{2}^{2} & =\bar{\pi}_{2} \Delta_{1}^{2} \\
& =\Delta_{1}^{2}-\bar{\pi}_{1} \frac{\Delta_{3}^{1} \Delta_{1}^{2}}{\Delta_{2}^{1}},
\end{aligned}
$$

where the second equality follows using (4.6). Rewriting the last equality leads to

$$
\bar{\pi}_{1}=\frac{\Delta_{2}^{1} \Delta_{1}^{2}}{\Delta_{1}^{1} \Delta_{1}^{2}+\Delta_{2}^{1} \Delta_{1}^{2}+\Delta_{2}^{1} \Delta_{2}^{2}} .
$$

It is immediate that $0<\bar{\pi}_{1}<1$.

By (4.7) we have $\bar{\pi}_{2}=\bar{\pi}_{1} \Delta_{2}^{2} / \Delta_{1}^{2}$, and we find that

$$
\bar{\pi}_{2}=\frac{\Delta_{2}^{1} \Delta_{2}^{2}}{\Delta_{1}^{1} \Delta_{1}^{2}+\Delta_{2}^{1} \Delta_{1}^{2}+\Delta_{2}^{1} \Delta_{2}^{2}} .
$$

Since $\bar{\pi}_{3}=1-\bar{\pi}_{1}-\bar{\pi}_{2}$, we find that

$$
\bar{\pi}_{3}=\frac{\Delta_{1}^{1} \Delta_{1}^{2}}{\Delta_{1}^{1} \Delta_{1}^{2}+\Delta_{2}^{1} \Delta_{1}^{2}+\Delta_{2}^{1} \Delta_{2}^{2}} .
$$

Obviously, it holds that $0<\bar{\pi}_{2}<1$ and $0<\bar{\pi}_{3}<1$. At this point we have established that there is at most one solution to (4.1)-(4.4). For there to be some solution, (4.3) should hold. Using the already derived expressions for $\bar{\pi}_{1}, \bar{\pi}_{2}$, and $\bar{\pi}_{3}$, we find that (4.3) holds if and only if

$$
\Delta_{1}^{1} \Delta_{1}^{2} \Delta_{1}^{3}=\Delta_{2}^{1} \Delta_{2}^{2} \Delta_{2}^{3} .
$$

This equation holds in degenerate cases only. It requires that the product over the players of the utility difference between their best and middle alternative be exactly equal to the product of the utility difference between the middle and the worst alternative.

A game $(u, \rho)$ with $\Delta_{1}^{1} \Delta_{1}^{2} \Delta_{1}^{3}=\Delta_{2}^{1} \Delta_{2}^{2} \Delta_{2}^{3}$ has many equilibria $(\bar{p}, \bar{q})$ of the type described in this subsection. All such equilibria can be constructed as follows. Let $\bar{\pi}$ be the uniquely determined probabilities by which the alternatives are implemented at equilibrium. Let $\lambda>0$ be such that, for $i=1,2,3, \lambda \bar{\pi}_{m_{i}} \leq \rho_{i}$. If player $i$ is selected as proposer, he proposes $x_{m_{i}}$ with probability $\lambda \bar{\pi}_{m_{i}} / \rho_{i}$ and $x_{b_{i}}$ with probability $1-\lambda \bar{\pi}_{m_{i}} / \rho_{i}$. The former proposal is accepted, the latter rejected. This construction ensures that (4.5) holds. The higher $\lambda$, the less delay before an outcome is accepted. The highest possible choice of $\lambda$ occurs when there is at least one player $i$ for which $\bar{p}_{m_{i}}^{i}=1$. In that case, the selection of player $i$ as a proposer leads to a proposal that is accepted for sure.

Summarizing, we have the following. Let utilities be such that $\Delta_{1}^{1} \Delta_{1}^{2} \Delta_{1}^{3}=\Delta_{2}^{1} \Delta_{2}^{2} \Delta_{2}^{3}$, so there is a unique solution $\bar{\pi} \gg 0$ to (4.1)-(4.4). Then the set of CPEs with all players making a proposal with probability one is given by

$$
\bar{p}=\left[\begin{array}{ccc}
0 & 0 & 0 \\
1-\bar{p}_{m_{1}}^{1} & 0 & \bar{p}_{m_{3}}^{3} \\
\bar{p}_{m_{1}}^{1} & 1-\bar{p}_{m_{2}}^{2} & 0 \\
0 & \bar{p}_{m_{2}}^{2} & 1-\bar{p}_{m_{3}}^{3}
\end{array}\right]
$$




$$
\bar{q}^{1}=\left[\begin{array}{l}
1 \\
0 \\
1 \\
1
\end{array}\right], \bar{q}^{2}=\left[\begin{array}{l}
1 \\
1 \\
0 \\
1
\end{array}\right], \text { and } \bar{q}^{3}=\left[\begin{array}{l}
1 \\
1 \\
1 \\
0
\end{array}\right]
$$

where $\bar{p}$ satisfies (4.5). The other CPEs are obtained by transferring part or all of the probability $1-\bar{p}_{m_{i}}^{i}$ by which $x_{b_{i}}$ is proposed by player $i$ to the option not to make a proposal, $x_{0}$.

In this CPE, each player randomizes between his security utility $u_{m_{i}}^{i}$, knowing it will be accepted for sure by player $i+1$, and some gamble among all three alternatives in case he either proposes his best alternative, knowing the latter will be rejected for sure, or does not make a proposal at all. In this gamble, at some future date either player $i-1$ proposes player $i$ 's best alternative and player $i$ accepts, or player $i+1$ may propose player $i$ 's worst alternative but since this is player $i-1$ 's best alternative the latter player accepts, or player $i$ proposes his middle alternative, which is accepted by player $i+1$. Notice that all equilibria are symmetric whenever the recognition probabilities $\left(\rho_{1}, \rho_{2}, \rho_{3}\right)$ are identical to $\left(\bar{\pi}_{2}, \bar{\pi}_{3}, \bar{\pi}_{1}\right)$, because the latter ensures that the probabilities of proposing the middle alternative are equal. In that case there is one equilibrium without delay, i.e. $p_{m_{i}}^{i}=1$ for all $i$. All other equilibria involve delay.

All CPEs $(\bar{p}, \bar{q})$ lead to the same equilibrium payoffs $\bar{z}$ given by $\bar{z}^{i}=u_{m_{i}}^{i}$. We have uniqueness in equilibrium utilities but multiplicity in the supporting equilibrium strategies. therefore, as mentioned before, the multiplicity of equilibria is inessential. Since also $\bar{v}_{i}^{i}=u_{m_{i}}^{i}=\bar{z}^{i}$, there is no advantage in being the proposer. The recognition probabilities $\rho$ do not influence the probability $\bar{\pi}_{j}$ that the bargaining process ends with outcome $x_{j}$. These probabilities depend on the utilities only.

We can summarize our findings regarding equilibrium existence in the following theorem.

ThEOREM 4.1 There is a $C P E(\bar{p}, \bar{q}) \in P \times Q$ with $\bar{p}_{m_{i}}^{i}>0, i=1,2,3$, if and only if $\Delta_{1}^{1} \Delta_{1}^{2} \Delta_{1}^{3}=\Delta_{2}^{1} \Delta_{2}^{2} \Delta_{2}^{3}$. In this case, there is a unique CPE with minimal expected delay, given by the solution $(\bar{p}, \bar{q})$ to (4.5), (4.8), and (4.9) with $\bar{p}_{m_{i}}^{i}=1$ for at least one player $i$, where $\left(\bar{\pi}_{1}, \bar{\pi}_{2}, \bar{\pi}_{3}\right)$ is the unique solution to (4.1)-(4.4). Other CPEs are obtained by proportionally lowering $\bar{p}_{m_{i}}^{i}$ across players $i$, as well as by shifting probability weight from $\bar{p}_{b_{i}}^{i}$ to $\bar{p}_{0}^{i}$. All CPEs induce the same equilibrium utilities, given by $\bar{z}^{i}=u_{m_{i}}^{i}, i=1,2,3$.

\subsection{Two Players with $\bar{p}_{m_{i}}^{i}>0$.}

Next we consider equilibria where one player, without loss of generality player 1, proposes his best outcome for sure, and the other two players put strictly positive weight on their middle outcome. We argue that no such equilibria exist.

Consider a game $(u, \rho) \in \mathcal{G}$ and let $\left(\left(\bar{p}_{b_{i}}^{i}, \bar{p}_{m_{i}}^{i}, \bar{q}_{m_{i}}^{i}, \bar{z}^{i}\right)_{i=1,2,3}, \bar{\pi}\right)$ be a solution to (3.15)(3.22) with $\bar{p}_{1}^{1}=1, \bar{p}_{3}^{2}>0$, and $\bar{p}_{1}^{3}>0$. By (3.15), $\left(1-\bar{q}_{2}^{1}\right) u_{2}^{2}+\bar{q}_{2}^{1} \bar{z}^{2} \leq u_{3}^{2}$, so $\bar{z}^{2} \leq u_{3}^{2}$. Suppose $\bar{z}^{2}<u_{3}^{2}$. Then $\bar{q}_{3}^{2}=0$ by $(3.17)$, so $\bar{p}_{1}^{3}=0$ by $(3.15)$, a contradiction to $\bar{p}_{1}^{3}>0$. It follows that $\bar{z}^{2}=u_{3}^{2}$. Now (3.15) implies $u_{3}^{2} \geq\left(1-\bar{q}_{2}^{1}\right) u_{2}^{2}+\bar{q}_{2}^{1} u_{3}^{2}$, so $\bar{q}_{2}^{1}=1$. It follows that if player 2 proposes his best alternative, it is rejected for sure. No other player ever proposes this alternative. The bargaining process never ends with outcome $x_{2}$, i.e. $\bar{\pi}_{2}=0$. This is a contradiction to $\bar{\pi}_{2}>0$. We conclude that there are no equilibria with the properties as 
stated in this subsection.

TheOrem 4.2 There is no $C P E(\bar{p}, \bar{q}) \in P \times Q$ with for some $i=1,2,3, \bar{p}_{m_{i}}^{i}=0$, $\bar{p}_{m_{i-1}}^{i-1}>0$, and $\bar{p}_{m_{i+1}}^{i+1}>0$.

\subsection{One Player with $\bar{p}_{m_{i}}^{i}>0$.}

Now we consider equilibria with two players proposing their best outcome for sure, and where one player, without loss of generality player 3 , puts strictly positive weight on his middle outcome.

Consider a game $(u, \rho) \in \mathcal{G}$ and let $\left(\left(\bar{p}_{b_{i}}^{i}, \bar{p}_{m_{i}}^{i}, \bar{q}_{m_{i}}^{i}, \bar{z}^{i}\right)_{i=1,2,3}, \bar{\pi}\right)$ be a solution to (3.15)(3.22) with $\bar{p}_{1}^{1}=1, \bar{p}_{2}^{2}=1$, and $\bar{p}_{1}^{3}>0$. So, player 1 proposes $x_{1}$, player 2 proposes $x_{2}$, and player 3 mixes over $x_{3}$ and $x_{1}$. To obtain $\bar{\pi}_{j}>0, j=1,2,3$, we must have $\bar{p}_{3}^{3}>0, \bar{q}_{2}^{1}<1$, and $\bar{q}_{3}^{2}<1$. By (3.15) and (3.16) we find

$$
\begin{aligned}
& \left(1-\bar{q}_{1}^{3}\right) u_{1}^{1}+\bar{q}_{1}^{3} \bar{z}^{1} \geq u_{2}^{1}, \\
& \left(1-\bar{q}_{2}^{1}\right) u_{2}^{2}+\bar{q}_{2}^{1} \bar{z}^{2} \geq u_{3}^{2}, \\
& \left(1-\bar{q}_{3}^{2}\right) u_{3}^{3}+\bar{q}_{3}^{2} \bar{z}^{3}=u_{1}^{3} .
\end{aligned}
$$

Since $\bar{q}_{3}^{2}<1$ and $u_{3}^{3}>u_{1}^{3}$, the equality implies $\bar{q}_{3}^{2} \in(0,1)$ and $\bar{z}^{3}<u_{1}^{3}$. It follows by (3.17) and (3.18) that $\bar{z}^{2}=u_{3}^{2}$. Since $\bar{z}^{3}<u_{1}^{3}$, we have $\bar{q}_{1}^{3}=0$ by (3.17), so the proposal of player 1 is accepted for sure. By $\bar{q}_{2}^{1}<1$ and (3.18), we also must have $\bar{z}^{1} \leq u_{2}^{1}$. There are now two possible cases. Case 1 where $\bar{z}^{1}=u_{2}^{1}$ and Case 2 with $\bar{z}^{1}<u_{2}^{1}$. In Case 2 we have $\bar{q}_{2}^{1}=0$ by (3.17).

\subsubsection{Case 1}

It holds that $(\bar{p}, \bar{q}) \in P \times Q$ is a CPE if and only if there is $\bar{\pi}$ such that

$$
\begin{aligned}
& \bar{p}=\left[\begin{array}{ccc}
0 & 0 & 0 \\
1 & 0 & 1-\bar{p}_{3}^{3} \\
0 & 1 & 0 \\
0 & 0 & \bar{p}_{3}^{3}
\end{array}\right] \\
& \bar{q}^{1}=\left[\begin{array}{c}
1 \\
0 \\
\bar{q}_{2}^{1} \\
1
\end{array}\right], \bar{q}^{2}=\left[\begin{array}{c}
1 \\
1 \\
0 \\
\bar{q}_{3}^{2}
\end{array}\right], \text { and } \bar{q}^{3}=\left[\begin{array}{l}
1 \\
0 \\
1 \\
0
\end{array}\right], \\
& \left(1-\bar{q}_{3}^{2}\right) u_{3}^{3}+\bar{q}_{3}^{2}\left(\bar{\pi}_{1} u_{1}^{3}+\bar{\pi}_{2} u_{2}^{3}+\bar{\pi}_{3} u_{3}^{3}\right)=u_{1}^{3}, \\
& \bar{\pi}_{1} u_{1}^{1}+\bar{\pi}_{2} u_{2}^{1}+\bar{\pi}_{3} u_{3}^{1}=u_{2}^{1}, \\
& \bar{\pi}_{1} u_{1}^{2}+\bar{\pi}_{2} u_{2}^{2}+\bar{\pi}_{3} u_{3}^{2}=u_{3}^{2}, \\
& \bar{\pi}_{1} u_{1}^{3}+\bar{\pi}_{2} u_{2}^{3}+\bar{\pi}_{3} u_{3}^{3}<u_{1}^{3}, \\
& \bar{\pi}_{1}+\bar{\pi}_{2}+\bar{\pi}_{3}=1, \\
& \bar{\pi}_{1}: \bar{\pi}_{2}: \bar{\pi}_{3}=\rho_{1}+\rho_{3}\left(1-\bar{p}_{3}^{3}\right): \rho_{2}\left(1-\bar{q}_{2}^{1}\right): \rho_{3} \bar{p}_{3}^{3}\left(1-\bar{q}_{3}^{2}\right),
\end{aligned}
$$


where $0<\bar{p}_{3}^{3}<1,0 \leq \bar{q}_{2}^{1}<1$, and $0<\bar{q}_{3}^{2}<1$.

Using the same derivation as in Subsection 4.1, it can be shown that there is a solution $\bar{\pi} \gg 0$ to the system (4.13)-(4.16) if and only if

$$
\Delta_{1}^{1} \Delta_{1}^{2} \Delta_{1}^{3}<\Delta_{2}^{1} \Delta_{2}^{2} \Delta_{2}^{3}
$$

Moreover, each specification of utilities satisfying (4.18) leads to a unique solution $\bar{\pi} \gg 0$ to (4.13)-(4.16). Indeed, as before it holds that

$$
\begin{aligned}
& \bar{\pi}_{1}=\frac{\Delta_{2}^{1} \Delta_{1}^{2}}{\Delta_{1}^{1} \Delta_{1}^{2}+\Delta_{2}^{1} \Delta_{1}^{2}+\Delta_{2}^{1} \Delta_{2}^{2}}, \\
& \bar{\pi}_{2}=\frac{\Delta_{2}^{1} \Delta_{2}^{2}}{\Delta_{1}^{1} \Delta_{1}^{2}+\Delta_{2}^{1} \Delta_{1}^{2}+\Delta_{2}^{1} \Delta_{2}^{2}}, \\
& \bar{\pi}_{3}=\frac{\Delta_{1}^{1} \Delta_{1}^{2}}{\Delta_{1}^{1} \Delta_{1}^{2}+\Delta_{2}^{1} \Delta_{1}^{2}+\Delta_{2}^{1} \Delta_{2}^{2}} .
\end{aligned}
$$

Inequality (4.18) specifies that the product over the players of the utility difference between their best and middle alternative should be less than the product of the utility difference between the middle and worst alternative.

Rewriting (4.12), we obtain

$$
\bar{q}_{3}^{2}=\frac{\Delta_{1}^{3}}{\bar{\pi}_{1} \Delta_{1}^{3}+\bar{\pi}_{2} \Delta_{3}^{3}},
$$

and substitution of the expressions for $\bar{\pi}_{1}$ and $\bar{\pi}_{2}$ results in

$$
\bar{q}_{3}^{2}=\frac{\Delta_{1}^{1} \Delta_{1}^{2} \Delta_{1}^{3}+\Delta_{2}^{1} \Delta_{1}^{2} \Delta_{1}^{3}+\Delta_{2}^{1} \Delta_{2}^{2} \Delta_{1}^{3}}{\Delta_{2}^{1} \Delta_{1}^{2} \Delta_{1}^{3}+\Delta_{2}^{1} \Delta_{2}^{2} \Delta_{1}^{3}+\Delta_{2}^{1} \Delta_{2}^{2} \Delta_{2}^{3}} .
$$

Notice that $0<\bar{q}_{3}^{2}<1$. By $(4.17)$ we have

$$
\frac{\Delta_{2}^{1}}{\Delta_{1}^{1}}=\frac{\rho_{1}+\rho_{3}\left(1-\bar{p}_{3}^{3}\right)}{\rho_{3} \bar{p}_{3}^{3}\left(1-\bar{q}_{3}^{2}\right)} \text {. }
$$

Substitution of the expression for $\bar{q}_{3}^{2}$ in the latter equation, and then solving for $\bar{p}_{3}^{3}$ results in

$$
\bar{p}_{3}^{3}=\frac{\rho_{1}+\rho_{3}}{\rho_{3}} \frac{\Delta_{1}^{1} \Delta_{1}^{2} \Delta_{1}^{3}+\Delta_{1}^{1} \Delta_{2}^{2} \Delta_{1}^{3}+\Delta_{1}^{1} \Delta_{2}^{2} \Delta_{2}^{3}}{\Delta_{1}^{1} \Delta_{2}^{2} \Delta_{1}^{3}+\Delta_{1}^{1} \Delta_{2}^{2} \Delta_{2}^{3}+\Delta_{2}^{1} \Delta_{2}^{2} \Delta_{2}^{3}} .
$$

Obviously, it holds that $\bar{p}_{3}^{3}>0$. Moreover, we have that $\bar{p}_{3}^{3}<1$ if and only if

$$
\frac{\rho_{1}}{\rho_{3}}<\frac{\Delta_{2}^{1} \Delta_{2}^{2} \Delta_{2}^{3}-\Delta_{1}^{1} \Delta_{1}^{2} \Delta_{1}^{3}}{\Delta_{1}^{1} \Delta_{1}^{2} \Delta_{1}^{3}+\Delta_{1}^{1} \Delta_{2}^{2} \Delta_{1}^{3}+\Delta_{1}^{1} \Delta_{2}^{2} \Delta_{2}^{3}}\left(<\frac{\Delta_{2}^{1}}{\Delta_{1}^{1}}\right) .
$$

By (4.17) we have

$$
\frac{\Delta_{1}^{2}}{\Delta_{2}^{2}}=\frac{\rho_{1}+\rho_{3}\left(1-\bar{p}_{3}^{3}\right)}{\rho_{2}\left(1-\bar{q}_{2}^{1}\right)} .
$$


We substitute the expression found for $\bar{p}_{3}^{3}$ and solve the resulting equation for $\bar{q}_{2}^{1}$, and obtain that

$$
1-\bar{q}_{2}^{1}=\frac{\rho_{1}+\rho_{3}}{\rho_{2}} \frac{\Delta_{2}^{1} \Delta_{2}^{2} \Delta_{2}^{3}-\Delta_{1}^{1} \Delta_{1}^{2} \Delta_{1}^{3}}{\Delta_{1}^{1} \Delta_{1}^{2} \Delta_{1}^{3}+\Delta_{1}^{1} \Delta_{1}^{2} \Delta_{2}^{3}+\Delta_{2}^{1} \Delta_{1}^{2} \Delta_{2}^{3}} .
$$

This expression is clearly strictly positive. It is less than or equal to one if and only if

$$
\rho_{2} \geq \frac{\Delta_{2}^{1} \Delta_{2}^{2} \Delta_{2}^{3}-\Delta_{1}^{1} \Delta_{1}^{2} \Delta_{1}^{3}}{\Delta_{1}^{1} \Delta_{1}^{2} \Delta_{2}^{3}+\Delta_{2}^{1} \Delta_{1}^{2} \Delta_{2}^{3}+\Delta_{2}^{1} \Delta_{2}^{2} \Delta_{2}^{3}} .
$$

Since all players $i$ propose their best outcome $x_{b_{i}}$ with strictly positive probability, and since $\bar{q}_{1}^{3}=0, \bar{q}_{2}^{1}<1$, and $\bar{q}_{3}^{2}<1$ implies that such a proposal is accepted with strictly positive probability, no player wants to use the option not to make a proposal. Finally, the equilibrium utilities satisfy $\bar{z}^{1}=u_{2}^{1}, \bar{z}^{2}=u_{3}^{2}$, and $\bar{z}^{3}<u_{1}^{3}$.

We summarize our findings in the following theorem.

TheOrem 4.3.1 There is a CPE $(\bar{p}, \bar{q}) \in P \times Q$ with $\bar{p}_{2}^{1}=0, \bar{p}_{3}^{2}=0, \bar{p}_{1}^{3}>0$, and $\bar{z}^{1} \geq u_{2}^{1}$ if and only if $\Delta_{1}^{1} \Delta_{1}^{2} \Delta_{1}^{3}<\Delta_{2}^{1} \Delta_{2}^{2} \Delta_{2}^{3}$ and $\rho$ is such that (4.21) and (4.23) are satisfied. In this case, such CPE is unique. It is given by (4.10), (4.11), (4.19), (4.20), and (4.22). The equilibrium utilities satisfy $\bar{z}^{1}=u_{2}^{1}, \bar{z}^{2}=u_{3}^{2}$, and $\bar{z}^{3}<u_{1}^{3}$.

For given utilities satisfying (4.18), (4.21) requires $\rho_{3}$ to be sufficiently high compared to $\rho_{1}$, and (4.23) requires $\rho_{2}$ to be sufficiently high.

To summarize, players 1 and 2 propose their best alternative whenever they are the recognized player. Player 1's best alternative is accepted for sure, whereas player 2's best alternative may be rejected with positive probability. By proposing his best alternative, this player chooses the risky option over his riskless security utility $u_{m_{2}}^{2}$. Player 3's proposal consists of randomizing between his best and middle alternative. Notice that, unlike the equilibrium of Theorem 4.1, players never use the option to refrain from making a proposal, i.e., $p_{0}^{i}=0, i=1,2$, 3. Since conditional equilibrium utilities satisfy $\bar{v}_{i}^{i}>\bar{z}^{i}, i=1,2$, 3 , each player enjoys an advantage whenever he is the recognized player. Moreover, conditional on being the recognized player, player 1 achieves his best alternative, player 2 is strictly better off compared to his security level, and player 3 is kept at his security level. In many bargaining models, the advantage to propose vanishes in taking the limit to the no discounting case. Here the advantage is present under no discounting. ${ }^{7}$

The equilibrium leads to a strictly positive expected delay. The reason is that player 3 is recognized with strictly positive probability and proposes $x_{3}$ with strictly positive probability. This proposal is rejected by both players with strictly positive probability. Player 1 always proposes $x_{1}$, which is accepted by player 3 . Player 2 always proposes $x_{2}$, which is accepted by player 1 with strictly probability $1-\bar{q}_{2}^{1}$ and is rejected by both players otherwise.

Using a straightforward relabeling of the players, we find fully symmetric results for equilibria with $\bar{p}_{2}^{2}=\bar{p}_{3}^{3}=1$ and player 1 mixing between $x_{1}$ and $x_{2}$, and equilibria with $\bar{p}_{1}^{1}=\bar{p}_{3}^{3}=1$ and player 2 mixing between $x_{2}$ and $x_{3}$.

\footnotetext{
${ }^{7}$ Houba (2008) reports a case with persistence of this advantage in bilateral alternating-offers bargaining.
} 


\subsubsection{Case 2}

It holds that $(\bar{p}, \bar{q}) \in P \times Q$ is a CPE if and only if there is $\bar{\pi}$ such that

$$
\begin{aligned}
& \bar{p}=\left[\begin{array}{ccc}
0 & 0 & 0 \\
1 & 0 & 1-\bar{p}_{3}^{3} \\
0 & 1 & 0 \\
0 & 0 & \bar{p}_{3}^{3}
\end{array}\right] \\
& \bar{q}^{1}=\left[\begin{array}{l}
1 \\
0 \\
0 \\
1
\end{array}\right], \bar{q}^{2}=\left[\begin{array}{c}
1 \\
1 \\
0 \\
\bar{q}_{3}^{2}
\end{array}\right], \text { and } \bar{q}^{3}=\left[\begin{array}{l}
1 \\
0 \\
1 \\
0
\end{array}\right], \\
& \left(1-\bar{q}_{3}^{2}\right) u_{3}^{3}+\bar{q}_{3}^{2}\left(\bar{\pi}_{1} u_{1}^{3}+\bar{\pi}_{2} u_{2}^{3}+\bar{\pi}_{3} u_{3}^{3}\right)=u_{1}^{3}, \\
& \bar{\pi}_{1} u_{1}^{1}+\bar{\pi}_{2} u_{2}^{1}+\bar{\pi}_{3} u_{3}^{1}<u_{2}^{1}, \\
& \bar{\pi}_{1} u_{1}^{2}+\bar{\pi}_{2} u_{2}^{2}+\bar{\pi}_{3} u_{3}^{2}=u_{3}^{2}, \\
& \bar{\pi}_{1} u_{1}^{3}+\bar{\pi}_{2} u_{2}^{3}+\bar{\pi}_{3} u_{3}^{3}<u_{1}^{3}, \\
& \bar{\pi}_{1}+\bar{\pi}_{2}+\bar{\pi}_{3}=1, \\
& \bar{\pi}_{1}: \bar{\pi}_{2}: \bar{\pi}_{3}=\rho_{1}+\rho_{3}\left(1-\bar{p}_{3}^{3}\right): \rho_{2}: \rho_{3} \bar{p}_{3}^{3}\left(1-\bar{q}_{3}^{2}\right),
\end{aligned}
$$

where $0<\bar{p}_{3}^{3}<1$ and $0<\bar{q}_{3}^{2}<1$.

We can rewrite (4.26)-(4.29) as

$$
\begin{aligned}
\Delta_{1}^{3}-\bar{q}_{3}^{2}\left(\bar{\pi}_{1} \Delta_{1}^{3}+\bar{\pi}_{2} \Delta_{3}^{3}\right) & =0 \\
\bar{\pi}_{1} \Delta_{1}^{1}-\bar{\pi}_{3} \Delta_{2}^{1} & <0 \\
-\bar{\pi}_{1} \Delta_{2}^{2}+\bar{\pi}_{2} \Delta_{1}^{2} & =0 \\
-\bar{\pi}_{2} \Delta_{2}^{3}+\bar{\pi}_{3} \Delta_{1}^{3} & <0 .
\end{aligned}
$$

We have a system $(4.30),(4.31),(4.32),(4.34)$ with five equations in the five unknowns $\bar{\pi}_{1}$, $\bar{\pi}_{2}, \bar{\pi}_{3}, \bar{p}_{3}^{3}$, and $\bar{q}_{3}^{2}$. Solving this system results in outcome probabilities

$$
\begin{aligned}
& \bar{\pi}_{1}=\frac{\Delta_{1}^{2} \Delta_{1}^{3}+\rho_{2} \Delta_{1}^{2} \Delta_{2}^{3}}{\Delta_{1}^{2} \Delta_{1}^{3}+\Delta_{2}^{2} \Delta_{1}^{3}+\Delta_{2}^{2} \Delta_{2}^{3}}, \\
& \bar{\pi}_{2}=\frac{\Delta_{2}^{2} \Delta_{1}^{3}+\rho_{2} \Delta_{2}^{2} \Delta_{2}^{3}}{\Delta_{1}^{2} \Delta_{1}^{3}+\Delta_{2}^{2} \Delta_{1}^{3}+\Delta_{2}^{2} \Delta_{2}^{3}}, \\
& \bar{\pi}_{3}=\frac{\Delta_{2}^{2} \Delta_{2}^{3}-\rho_{2} \Delta_{3}^{2} \Delta_{2}^{3}}{\Delta_{1}^{2} \Delta_{1}^{3}+\Delta_{2}^{2} \Delta_{1}^{3}+\Delta_{2}^{2} \Delta_{2}^{3}},
\end{aligned}
$$

and equilibrium action probabilities

$$
\begin{aligned}
\bar{p}_{3}^{3} & =\frac{\rho_{1} \Delta_{2}^{2}-\rho_{2} \Delta_{1}^{2}+\rho_{3} \Delta_{2}^{2}}{\rho_{3} \Delta_{2}^{2}}, \\
\bar{q}_{3}^{2} & =\frac{\Delta_{1}^{3}}{\Delta_{1}^{3}+\rho_{2} \Delta_{2}^{3}} .
\end{aligned}
$$

It is immediate that the solution satisfies $0<\bar{q}_{3}^{2}<1$. 
The inequality (4.33) is equivalent to

$$
\rho_{2}<\frac{\Delta_{2}^{1} \Delta_{2}^{2} \Delta_{2}^{3}-\Delta_{1}^{1} \Delta_{1}^{2} \Delta_{1}^{3}}{\Delta_{1}^{1} \Delta_{1}^{2} \Delta_{2}^{3}+\Delta_{2}^{1} \Delta_{1}^{2} \Delta_{2}^{3}+\Delta_{2}^{1} \Delta_{2}^{2} \Delta_{2}^{3}}\left(<\frac{\Delta_{2}^{2}}{\Delta_{1}^{2}+\Delta_{2}^{2}}\right) .
$$

The inequality (4.35) is always satisfied.

The requirement $\bar{p}_{3}^{3}>0$ is equivalent to $\rho_{2}<\Delta_{2}^{2} / \Delta_{3}^{2}$, which implies $\bar{\pi}_{3}>0$. This requirement follows from (4.38). The requirement $\bar{p}_{3}^{3}<1$ is equivalent to

$$
\frac{\rho_{1}}{\rho_{2}}<\frac{\Delta_{1}^{2}}{\Delta_{2}^{2}}
$$

Since all players $i$ propose their best outcome $x_{b_{i}}$ with strictly positive probability, and since $\bar{q}_{1}^{3}=0, \bar{q}_{2}^{1}=0$, and $\bar{q}_{3}^{2}<1$ implies that such a proposal is accepted with strictly positive probability, no player wants to use the option not to make a proposal. We summarize our findings in the following theorem.

TheOREM 4.3.2 There is a $C P E(\bar{p}, \bar{q}) \in P \times Q$ with $\bar{p}_{2}^{1}=0, \bar{p}_{3}^{2}=0, \bar{p}_{1}^{3}>0$, and $\bar{z}^{1}<u_{2}^{1}$ if and only if $\Delta_{1}^{1} \Delta_{1}^{2} \Delta_{1}^{3}<\Delta_{2}^{1} \Delta_{2}^{2} \Delta_{2}^{3}$ and $\rho$ is such that (4.38) and (4.39) are satisfied. In this case, a CPE is unique. It is given by (4.24), (4.25), (4.36), and (4.37). Equilibrium utilities satisfy $\bar{z}^{1}<u_{2}^{1}, \bar{z}^{2}=u_{3}^{2}$, and $\bar{z}^{3}<u_{1}^{3}$.

For given utilities satisfying (4.18), (4.38) requires $\rho_{2}$ to be sufficiently low. It complements (4.23) which implies that equilibria as in Case 1 cannot coexist with those as in Case 2. Inequality (4.39) requires $\rho_{1}$ to be sufficiently low compared to $\rho_{2}$. Notice that, like the equilibrium of Theorem 4.3.1, the option not to make a proposal cannot be chosen with any positive probability.

The equilibrium leads to a strictly positive expected delay. The reason is that player 3 is recognized with strictly positive probability, proposes $x_{3}$ with strictly positive probability, which is rejected by both players with strictly positive probability. The proposals of players 1 and 2 are accepted for sure. Similar to the previous case, all three players have an advantage to propose. Conditional on being the recognized player, player 3 cannot do better than getting the utility of his middle alternative. Conditional on being the proposer, both player 1 and player 2 achieve the utility of the best alternative.

By a relabeling of the players, we find fully symmetric results for equilibria with $\bar{p}_{2}^{2}=$ $\bar{p}_{3}^{3}=1$ and player 1 mixing between $x_{1}$ and $x_{2}$, and equilibria with $\bar{p}_{1}^{1}=\bar{p}_{3}^{3}=1$ and player 2 mixing between $x_{2}$ and $x_{3}$.

\subsection{No Player with $\bar{p}_{m_{i}}^{i}>0$.}

We finally consider equilibria where all players propose their best outcome for sure. Let $\left(\bar{p}_{b_{i}}^{i}, \bar{p}_{m_{i}}^{i}, \bar{q}_{m_{i}}^{i}, \bar{z}^{i}\right)$ be a solution to $(3.15)-(3.22)$ with $\bar{p}_{1}^{1}=\bar{p}_{2}^{2}=\bar{p}_{3}^{3}=1$. To obtain $\bar{\pi}_{j}>0$, $j=1,2,3$, we must have $\bar{q}_{1}^{3}<1, \bar{q}_{2}^{1}<1$, and $\bar{q}_{3}^{2}<1$. If follows from (3.18) that $\bar{z}^{1} \leq u_{2}^{1}$, $\bar{z}^{2} \leq u_{3}^{2}$, and $\bar{z}^{3} \leq u_{1}^{3}$. Since all players propose their best outcome with strictly positive probability, and since such a proposal is accepted with strictly positive probability, no player wants to use the option not to make a proposal. We distinguish four possible cases of interest. In Case 1 , there are three players with $\bar{z}^{i}=u_{m_{i}}^{i}$, in Case 2 there are two such players, without loss of generality, players 1 and 2 , in Case 3 there is one such player, without loss of generality player 1 , and in Case 4 all players have $\bar{z}^{i}<u_{m_{i}}^{i}$. 


\subsubsection{Case 1}

It holds that $(\bar{p}, \bar{q}) \in P \times Q$ is a CPE if and only if there is $\bar{\pi}$ such that

$$
\begin{aligned}
& \bar{p}=\left[\begin{array}{lll}
0 & 0 & 0 \\
1 & 0 & 0 \\
0 & 1 & 0 \\
0 & 0 & 1
\end{array}\right] \\
& \bar{q}^{1}=\left[\begin{array}{c}
1 \\
0 \\
\bar{q}_{2}^{1} \\
1
\end{array}\right], \bar{q}^{2}=\left[\begin{array}{c}
1 \\
1 \\
0 \\
\bar{q}_{3}^{2}
\end{array}\right], \text { and } \bar{q}^{3}=\left[\begin{array}{c}
1 \\
\bar{q}_{1}^{3} \\
1 \\
0
\end{array}\right], \\
& \bar{\pi}_{1} u_{1}^{1}+\bar{\pi}_{2} u_{2}^{1}+\bar{\pi}_{3} u_{3}^{1}=u_{2}^{1}, \\
& \bar{\pi}_{1} u_{1}^{2}+\bar{\pi}_{2} u_{2}^{2}+\bar{\pi}_{3} u_{3}^{2}=u_{3}^{2}, \\
& \bar{\pi}_{1} u_{1}^{3}+\bar{\pi}_{2} u_{2}^{3}+\bar{\pi}_{3} u_{3}^{3}=u_{1}^{3}, \\
& \bar{\pi}_{1}+\bar{\pi}_{2}+\bar{\pi}_{3}=1, \\
& \bar{\pi}_{1}: \bar{\pi}_{2}: \bar{\pi}_{3}=\rho_{1}\left(1-\bar{q}_{1}^{3}\right): \rho_{2}\left(1-\bar{q}_{2}^{1}\right): \rho_{3}\left(1-\bar{q}_{3}^{2}\right) .
\end{aligned}
$$

As in Section 4.1 we obtain that

$$
\begin{aligned}
& \Delta_{1}^{1} \Delta_{1}^{2} \Delta_{1}^{3}=\Delta_{2}^{1} \Delta_{2}^{2} \Delta_{2}^{3}, \\
& \bar{\pi}_{1}=\frac{\Delta_{2}^{1} \Delta_{1}^{2}}{\Delta_{1}^{1} \Delta_{1}^{2}+\Delta_{2}^{1} \Delta_{1}^{2}+\Delta_{2}^{1} \Delta_{2}^{2}}, \\
& \bar{\pi}_{2}=\frac{\Delta_{2}^{1} \Delta_{2}^{2}}{\Delta_{1}^{1} \Delta_{1}^{2}+\Delta_{2}^{1} \Delta_{1}^{2}+\Delta_{2}^{1} \Delta_{2}^{2}}, \\
& \bar{\pi}_{3}=\frac{\Delta_{1}^{1} \Delta_{1}^{2}}{\Delta_{1}^{1} \Delta_{1}^{2}+\Delta_{2}^{1} \Delta_{1}^{2}+\Delta_{2}^{1} \Delta_{2}^{2}} .
\end{aligned}
$$

The CPE is not unique. Let $\lambda>0$ be such that, for $i=1,2,3, \lambda \bar{\pi}_{m_{i}} \leq \rho_{m_{i}}$. If player $i$ has to respond to the proposal $x_{m_{i}}$, he accepts with probability $\lambda \bar{\pi}_{m_{i}} / \rho_{m_{i}}>0$ and rejects with probability $\bar{q}_{m_{i}}^{i}=1-\lambda \bar{\pi}_{m_{i}} / \rho_{m_{i}}<1$. This construction ensures that (4.42) holds. The higher $\lambda$, the less delay before an outcome is accepted. The highest possible choice of $\lambda$ occurs when there is at least one player $i$ for which $\bar{q}_{m_{i}}^{i}=0$. In that case, selection of player $i+1$ as proposer leads to a proposal that is accepted for sure. Note that $\lambda=0$ would violate $\bar{\pi}_{m_{i}}>0$. The set of CPEs is not closed. The no discounting case differs in this respect from the discounting case where the set of equilibria is compact. This conforms to Herings and Houba (2010) who report for a general class of bargaining models that the set of CPEs may fail to be compact in the no discounting case.

By definition of this case, the equilibrium utilities satisfy $\bar{z}^{i}=u_{m_{i}}^{i}, i=1,2,3$. Since also $\bar{q}_{m_{i}}^{i}<1$ and $\bar{z}^{i}=u_{m_{i}}^{i}$, the conditional equilibrium utilities satisfy $\bar{v}_{i}^{i} \in\left(u_{m_{i}}^{i}, u_{b_{i}}^{i}\right)$, $i=1,2,3$. We conclude that there is an advantage in becoming the recognized player and that a recognized player does strictly better than his security level $u_{m_{i}}^{i}$. 
THEOREM 4.4.1 There is a $C P E(\bar{p}, \bar{q}) \in P \times Q$ with $\bar{p}_{m_{i}}^{i}=0, i=1,2,3$, and $\bar{z}^{i} \geq u_{m_{i}}^{i}$, $i=1,2,3$, if and only if $\Delta_{1}^{1} \Delta_{1}^{2} \Delta_{1}^{3}=\Delta_{2}^{1} \Delta_{2}^{2} \Delta_{2}^{3}$. In this case, there is a unique CPE with minimal delay. It is given by the solution $(\bar{p}, \bar{q})$ to (4.40), (4.41), and (4.42) with $\bar{q}_{m_{i}}^{i}=0$ for at least one player $i$, where $\left(\bar{\pi}_{1}, \bar{\pi}_{2}, \bar{\pi}_{3}\right)$ is defined in (4.44)-(4.46). Other CPEs are obtained by proportionally lowering $1-\bar{q}_{m_{i}}^{i}$ across players $i$. All CPEs induce the same equilibrium utilities, given by $\bar{z}^{i}=u_{m_{i}}^{i}, i=1,2,3$.

\subsubsection{Case 2}

It holds that $(\bar{p}, \bar{q}) \in P \times Q$ is a CPE if and only if there is $\bar{\pi}$ such that

$$
\begin{aligned}
& \bar{p}=\left[\begin{array}{lll}
0 & 0 & 0 \\
1 & 0 & 0 \\
0 & 1 & 0 \\
0 & 0 & 1
\end{array}\right] \\
& \bar{q}^{1}=\left[\begin{array}{c}
1 \\
0 \\
\bar{q}_{2}^{1} \\
1
\end{array}\right], \bar{q}^{2}=\left[\begin{array}{c}
1 \\
1 \\
0 \\
\bar{q}_{3}^{2}
\end{array}\right], \text { and } \bar{q}^{3}=\left[\begin{array}{l}
1 \\
0 \\
1 \\
0
\end{array}\right], \\
& \left(1-\bar{q}_{3}^{2}\right) u_{3}^{3}+\bar{q}_{3}^{2}\left(\bar{\pi}_{1} u_{1}^{3}+\bar{\pi}_{2} u_{2}^{3}+\bar{\pi}_{3} u_{3}^{3}\right) \geq u_{1}^{3}, \\
& \bar{\pi}_{1} u_{1}^{1}+\bar{\pi}_{2} u_{2}^{1}+\bar{\pi}_{3} u_{3}^{1}=u_{2}^{1}, \\
& \bar{\pi}_{1} u_{1}^{2}+\bar{\pi}_{2} u_{2}^{2}+\bar{\pi}_{3} u_{3}^{2}=u_{3}^{2}, \\
& \bar{\pi}_{1} u_{1}^{3}+\bar{\pi}_{2} u_{2}^{3}+\bar{\pi}_{3} u_{3}^{3}<u_{1}^{3}, \\
& \bar{\pi}_{1}+\bar{\pi}_{2}+\bar{\pi}_{3}=1, \\
& \bar{\pi}_{1}: \bar{\pi}_{2}: \bar{\pi}_{3}=\rho_{1}: \rho_{2}\left(1-\bar{q}_{2}^{1}\right): \rho_{3}\left(1-\bar{q}_{3}^{2}\right) .
\end{aligned}
$$

As in Subsection 4.3.1 we obtain that

$$
\Delta_{1}^{1} \Delta_{1}^{2} \Delta_{1}^{3}<\Delta_{2}^{1} \Delta_{2}^{2} \Delta_{2}^{3}
$$

$$
\begin{aligned}
& \bar{\pi}_{1}=\frac{\Delta_{2}^{1} \Delta_{1}^{2}}{\Delta_{1}^{1} \Delta_{1}^{2}+\Delta_{2}^{1} \Delta_{1}^{2}+\Delta_{2}^{1} \Delta_{2}^{2}}, \\
& \bar{\pi}_{2}=\frac{\Delta_{2}^{1} \Delta_{2}^{2}}{\Delta_{1}^{1} \Delta_{1}^{2}+\Delta_{2}^{1} \Delta_{1}^{2}+\Delta_{2}^{1} \Delta_{2}^{2}}, \\
& \bar{\pi}_{3}=\frac{\Delta_{1}^{1} \Delta_{1}^{2}}{\Delta_{1}^{1} \Delta_{1}^{2}+\Delta_{2}^{1} \Delta_{1}^{2}+\Delta_{2}^{1} \Delta_{2}^{2}} .
\end{aligned}
$$

From (4.50) it then follows that

$$
\begin{aligned}
& \bar{q}_{2}^{1}=1-\frac{\rho_{1}}{\rho_{2}} \frac{\Delta_{2}^{2}}{\Delta_{1}^{2}}, \\
& \bar{q}_{3}^{2}=1-\frac{\rho_{1}}{\rho_{3}} \frac{\Delta_{1}^{1}}{\Delta_{2}^{1}} .
\end{aligned}
$$


To satisfy (4.49) we need that

$$
\frac{\rho_{1}}{\rho_{3}} \geq \frac{\Delta_{2}^{1} \Delta_{2}^{2} \Delta_{2}^{3}-\Delta_{1}^{1} \Delta_{1}^{2} \Delta_{1}^{3}}{\Delta_{1}^{1} \Delta_{1}^{2} \Delta_{1}^{3}+\Delta_{1}^{1} \Delta_{2}^{2} \Delta_{1}^{3}+\Delta_{1}^{1} \Delta_{2}^{2} \Delta_{2}^{3}}\left(\leq \frac{\Delta_{2}^{1}}{\Delta_{1}^{1}}\right)
$$

The requirements that $\bar{q}_{2}^{1} \geq 0$ and $\bar{q}_{3}^{2} \geq 0$ lead to

$$
\begin{aligned}
& \frac{\rho_{1}}{\rho_{2}} \leq \frac{\Delta_{1}^{2}}{\Delta_{2}^{2}}, \\
& \frac{\rho_{3}}{\rho_{1}} \geq \frac{\Delta_{1}^{1}}{\Delta_{2}^{1}} .
\end{aligned}
$$

By definition of the case, the equilibrium utilities satisfy $\bar{z}^{1}=u_{m_{1}}^{1}, \bar{z}^{2}=u_{m_{2}}^{2}$, and $\bar{z}^{3}<u_{m_{3}}^{3}$. For $i=1,2, q_{b_{i}}^{i-1}<1$ and $\bar{z}^{i}=u_{m_{i}}^{i}$ imply that the conditional equilibrium utilities satisfy $\bar{v}_{i}^{i}>u_{m_{i}}^{i}$. Since $\bar{z}^{3}<u_{m_{3}}^{3}$, it follows that player 3 has an advantage to propose.

We can summarize our findings in the following theorem.

TheOrem 4.4.2 There is a $C P E(\bar{p}, \bar{q}) \in P \times Q$ with $\bar{p}_{m_{i}}^{i}=0, i=1,2,3, \bar{z}^{1} \geq u_{2}^{1}$, $\bar{z}^{2} \geq u_{3}^{2}$, and $\bar{z}^{3}<u_{1}^{3}$ if and only if $\Delta_{1}^{1} \Delta_{1}^{2} \Delta_{1}^{3}<\Delta_{2}^{1} \Delta_{2}^{2} \Delta_{2}^{3}$ and $\rho$ is such that (4.54), (4.55), and (4.56) are satisfied. In this case, there is a unique CPE. It is given by (4.47), (4.48), (4.52), and (4.53). The equilibrium utilities satisfy $\bar{z}^{1}=u_{2}^{1}, \bar{z}^{2}=u_{3}^{2}$, and $\bar{z}^{3}<u_{1}^{3}$.

For given utilities satisfying (4.51), (4.54) requires $\rho_{1}$ to be sufficiently high compared to $\rho_{3}$, and (4.56) requires $\rho_{1}$ to be sufficiently low. Moreover, $\rho_{1}$ should be sufficiently low compared to $\rho_{2}$ by (4.55). Notice that, unlike the equilibrium of Theorem 4.1, the option not to make a proposal cannot be chosen with any positive probability.

By (4.50), the equilibrium does not involve delay if and only if $\rho_{i}$ is equal to $\bar{\pi}_{i}$ for ALL $i=1,2,3$.

By a relabeling of the players, we obtain fully symmetric results for equilibria with $\bar{p}_{m_{i}}^{i}=0, i=1,2,3, \bar{z}^{1} \geq u_{2}^{1}, \bar{z}^{2}<u_{3}^{2}$, and $\bar{z}^{3} \geq u_{1}^{3}$, and for equilibria with $\bar{p}_{m_{i}}^{i}=0$, $i=1,2,3, \bar{z}^{1}<u_{2}^{1}, \bar{z}^{2} \geq u_{3}^{2}$, and $\bar{z}^{3} \geq u_{1}^{3}$.

\subsubsection{Case 3}

It holds that $(\bar{p}, \bar{q}) \in P \times Q$ is a CPE if and only if there is $\bar{\pi}$ such that

$$
\begin{aligned}
& \bar{p}=\left[\begin{array}{lll}
0 & 0 & 0 \\
1 & 0 & 0 \\
0 & 1 & 0 \\
0 & 0 & 1
\end{array}\right] \\
& \bar{q}^{1}=\left[\begin{array}{c}
1 \\
0 \\
\bar{q}_{2}^{1} \\
1
\end{array}\right], \bar{q}^{2}=\left[\begin{array}{l}
1 \\
1 \\
0 \\
0
\end{array}\right], \text { and } \bar{q}^{3}=\left[\begin{array}{l}
1 \\
0 \\
1 \\
0
\end{array}\right], \\
& \left(1-\bar{q}_{2}^{1}\right) u_{2}^{2}+\bar{q}_{2}^{1}\left(\bar{\pi}_{1} u_{1}^{2}+\bar{\pi}_{2} u_{2}^{2}+\bar{\pi}_{3} u_{3}^{2}\right) \geq u_{3}^{2},
\end{aligned}
$$




$$
\begin{aligned}
\bar{\pi}_{1} u_{1}^{1}+\bar{\pi}_{2} u_{2}^{1}+\bar{\pi}_{3} u_{3}^{1}=u_{2}^{1}, \\
\bar{\pi}_{1} u_{1}^{2}+\bar{\pi}_{2} u_{2}^{2}+\bar{\pi}_{3} u_{3}^{2}<u_{3}^{2}, \\
\bar{\pi}_{1} u_{1}^{3}+\bar{\pi}_{2} u_{2}^{3}+\bar{\pi}_{3} u_{3}^{3}<u_{1}^{3}, \\
\bar{\pi}_{1}+\bar{\pi}_{2}+\bar{\pi}_{3}=1, \\
\bar{\pi}_{1}: \bar{\pi}_{2}: \bar{\pi}_{3}=\rho_{1}: \rho_{2}\left(1-\bar{q}_{2}^{1}\right): \rho_{3} .
\end{aligned}
$$

Rewriting (4.60) and using (4.63), we find that

$$
\rho_{3}=\rho_{1} \frac{\Delta_{1}^{1}}{\Delta_{2}^{1}}
$$

It follows that Case 3 admits consistent subgame perfect equilibria in degenerate cases only, more precisely, when (4.64) holds. In these degenerate cases, there is a continuum of consistent subgame perfect equilibria, inducing a continuum of equilibrium utilities for players 2 and 3. We parametrize the equilibria by means of the strictly positive real number $\lambda$ and using (4.63) we write

$$
\begin{aligned}
& \bar{\pi}_{1}=\lambda \rho_{1}, \\
& \bar{\pi}_{3}=\lambda \rho_{1} \frac{\Delta_{1}^{1}}{\Delta_{2}^{1}} .
\end{aligned}
$$

Suppose by means of contradiction that $\lambda<1$. Using (4.63), we find that

$$
\begin{aligned}
& \bar{\pi}_{1}=\lambda \rho_{1}<\rho_{1}, \\
& \bar{\pi}_{2}=\frac{\bar{\pi}_{1} \rho_{2}\left(1-\bar{q}_{2}^{1}\right)}{\rho_{1}}=\lambda \rho_{2}\left(1-\bar{q}_{2}^{1}\right)<\rho_{2}, \\
& \bar{\pi}_{3}=\frac{\bar{\pi}_{1} \rho_{3}}{\rho_{1}}=\lambda \rho_{3}<\rho_{3} .
\end{aligned}
$$

We obtain the contradiction $1=\bar{\pi}_{1}+\bar{\pi}_{2}+\bar{\pi}_{3}<\rho_{1}+\rho_{2}+\rho_{3}=1$. Consequently, we have shown that $\lambda \geq 1$.

Since $\rho_{1}+\rho_{2}+\rho_{3}=1$ and $\bar{\pi}_{1}+\bar{\pi}_{2}+\bar{\pi}_{3}=1$, we have

$$
\begin{aligned}
& \rho_{2}=1-\rho_{1} \frac{\Delta_{1}^{1}+\Delta_{2}^{1}}{\Delta_{2}^{1}}, \\
& \bar{\pi}_{2}=1-\lambda \rho_{1} \frac{\Delta_{1}^{1}+\Delta_{2}^{1}}{\Delta_{2}^{1}} .
\end{aligned}
$$

Using (4.63), we have

$$
\bar{q}_{2}^{1}=\frac{\lambda \Delta_{2}^{1}-\Delta_{2}^{1}}{\lambda \Delta_{2}^{1}-\lambda \rho_{1} \Delta_{3}^{1}}
$$

The denominator of (4.65) is strictly positive if and only if $\rho_{1}<\Delta_{2}^{1} / \Delta_{3}^{1}$. The inequalities in (4.61) and (4.59) are satisfied if and only if

$$
\frac{\Delta_{2}^{1} \Delta_{1}^{2}}{\Delta_{1}^{1} \Delta_{1}^{2}+\Delta_{2}^{1} \Delta_{1}^{2}+\Delta_{2}^{1} \Delta_{2}^{2}}<\lambda \rho_{1} \leq \frac{\Delta_{2}^{1} \Delta_{1}^{2}+\rho_{1} \Delta_{2}^{1} \Delta_{2}^{2}}{\Delta_{1}^{1} \Delta_{1}^{2}+\Delta_{2}^{1} \Delta_{1}^{2}+\Delta_{2}^{1} \Delta_{2}^{2}}\left(<\frac{\Delta_{2}^{1}}{\Delta_{1}^{1}+\Delta_{2}^{1}}\right) .
$$


The inequality in (4.66) in parentheses implies that $\bar{q}_{2}^{1}$ is strictly less than one, and that $\bar{\pi}_{2}$ is strictly positive.

The inequality (4.62) is satisfied if and only if

$$
\lambda \rho_{1}<\frac{\Delta_{2}^{1} \Delta_{2}^{3}}{\Delta_{1}^{1} \Delta_{1}^{3}+\Delta_{1}^{1} \Delta_{2}^{3}+\Delta_{2}^{1} \Delta_{2}^{3}} .
$$

The first inequality of (4.66) together with (4.67) imply that

$$
\Delta_{1}^{1} \Delta_{1}^{2} \Delta_{1}^{3}<\Delta_{2}^{1} \Delta_{2}^{2} \Delta_{2}^{3} .
$$

There is some $\lambda \geq 1$ such that (4.66) and (4.67) are satisfied if and only if $\Delta_{1}^{1} \Delta_{1}^{2} \Delta_{1}^{3}<$ $\Delta_{2}^{1} \Delta_{2}^{2} \Delta_{2}^{3}$ and

$$
\rho_{1}<\frac{\Delta_{2}^{1} \Delta_{2}^{3}}{\Delta_{1}^{1} \Delta_{1}^{3}+\Delta_{1}^{1} \Delta_{2}^{3}+\Delta_{2}^{1} \Delta_{2}^{3}} .
$$

The lowest possible value of $\lambda \geq 1$ such that (4.66) and (4.67) are satisfied is given by

$$
\max \left\{1, \frac{1}{\rho_{1}} \frac{\Delta_{2}^{1} \Delta_{1}^{2}}{\Delta_{1}^{1} \Delta_{1}^{2}+\Delta_{2}^{1} \Delta_{1}^{2}+\Delta_{2}^{1} \Delta_{2}^{2}}\right\} .
$$

We can summarize our findings in the following theorem.

TheOREM 4.4.3 There is a $C P E(\bar{p}, \bar{q}) \in P \times Q$ with $\bar{p}_{m_{i}}^{i}=0, i=1,2,3, \bar{z}^{1} \geq u_{2}^{1}$, $\bar{z}^{2}<u_{3}^{2}$, and $\bar{z}^{3}<u_{1}^{3}$ if and only if $\Delta_{1}^{1} \Delta_{1}^{2} \Delta_{1}^{3}<\Delta_{2}^{1} \Delta_{2}^{2} \Delta_{2}^{3}, \rho_{3}=\rho_{1} \Delta_{1}^{1} / \Delta_{2}^{1}$, and $\rho_{1}$ satisfies (4.68). In this case there is a continuum of consistent subgame perfect equilibria. Any $\lambda \geq 1$ satisfying (4.66) and (4.67) induces a consistent subgame perfect equilibrium given by (4.57), (4.58), and (4.65). Equilibrium utilities depend on $\lambda$ and satisfy $\bar{z}^{1}=u_{2}^{1}$, $\bar{z}^{2}<u_{3}^{2}$, and $\bar{z}^{3}<u_{1}^{3}$.

Notice that, unlike the equilibrium of Theorem 4.1, the option not to make a proposal cannot be chosen with any positive probability. The equilibrium does not involve delay if and only if $\lambda=1$. Whenever $\rho_{1}<\Delta_{2}^{1} \Delta_{1}^{2} /\left(\Delta_{1}^{1} \Delta_{1}^{2}+\Delta_{2}^{1} \Delta_{1}^{2}+\Delta_{2}^{1} \Delta_{2}^{2}\right)$, the lowest possible choice for $\lambda$ strictly exceeds 1 , and delay cannot be avoided.

Fully symmetric results hold for equilibria with $\bar{p}_{m_{i}}^{i}=0, i=1,2,3, \bar{z}^{1}<u_{2}^{1}, \bar{z}^{2} \geq u_{3}^{2}$, and $\bar{z}^{3}<u_{1}^{3}$, and for equilibria with $\bar{p}_{m_{i}}^{i}=0, i=1,2,3, \bar{z}^{1}<u_{2}^{1}, \bar{z}^{2}<u_{3}^{2}$, and $\bar{z}^{3} \geq u_{1}^{3}$.

\subsubsection{Case 4}

It holds that $(\bar{p}, \bar{q}) \in P \times Q$ is a CPE if and only if there is $\bar{\pi}$ such that

$$
\begin{aligned}
& \bar{p}=\left[\begin{array}{lll}
0 & 0 & 0 \\
1 & 0 & 0 \\
0 & 1 & 0 \\
0 & 0 & 1
\end{array}\right] \\
& \bar{q}^{1}=\left[\begin{array}{l}
1 \\
0 \\
0 \\
1
\end{array}\right], \bar{q}^{2}=\left[\begin{array}{l}
1 \\
1 \\
0 \\
0
\end{array}\right], \text { and } \bar{q}^{3}=\left[\begin{array}{l}
1 \\
0 \\
1 \\
0
\end{array}\right],
\end{aligned}
$$




$$
\begin{aligned}
& \bar{\pi}_{1} u_{1}^{1}+\bar{\pi}_{2} u_{2}^{1}+\bar{\pi}_{3} u_{3}^{1}<u_{2}^{1}, \\
& \bar{\pi}_{1} u_{1}^{2}+\bar{\pi}_{2} u_{2}^{2}+\bar{\pi}_{3} u_{3}^{2}<u_{3}^{2}, \\
& \bar{\pi}_{1} u_{1}^{3}+\bar{\pi}_{2} u_{2}^{3}+\bar{\pi}_{3} u_{3}^{3}<u_{1}^{3}, \\
& \bar{\pi}_{1}+\bar{\pi}_{2}+\bar{\pi}_{3}=1, \\
& \bar{\pi}_{1}: \bar{\pi}_{2}: \bar{\pi}_{3}=\rho_{1}: \rho_{2}: \rho_{3} .
\end{aligned}
$$

The equalities in (4.75) immediately lead to the conclusion that $\bar{\pi}_{1}=\rho_{1}, \bar{\pi}_{2}=\rho_{2}$, and $\bar{\pi}_{3}=\rho_{3}$. The inequalities in (4.72)-(4.74) are equivalent to the following conditions:

$$
\begin{aligned}
& \frac{\rho_{3}}{\rho_{1}}>\frac{\Delta_{1}^{1}}{\Delta_{2}^{1}}, \\
& \frac{\rho_{1}}{\rho_{2}}>\frac{\Delta_{1}^{2}}{\Delta_{2}^{2}}, \\
& \frac{\rho_{2}}{\rho_{3}}>\frac{\Delta_{1}^{3}}{\Delta_{2}^{3}} .
\end{aligned}
$$

It is immediate to verify that (4.76)-(4.78) imply that $\Delta_{1}^{1} \Delta_{1}^{2} \Delta_{1}^{3}<\Delta_{2}^{1} \Delta_{2}^{2} \Delta_{2}^{3}$.

By definition of the case, the equilibrium utilities satisfy $\bar{z}^{1}<u_{m_{1}}^{1}, \bar{z}^{2}<u_{m_{2}}^{2}$ and $\bar{z}^{3}<$ $u_{m_{3}}^{3}$. Since each player accepts his middle alternative for sure, the conditional equilibrium utilities satisfy $\bar{v}_{1}^{1}=u_{1}^{1}, \bar{v}_{2}^{2}=u_{2}^{2}$ and $\bar{v}_{3}^{3}=u_{3}^{3}$. Therefore, each player has an advantage to propose and, as the recognized player, each player can realize his best alternative for sure.

We can summarize our findings in the following theorem.

Theorem 4.4.4 There is a CPE $(\bar{p}, \bar{q}) \in P \times Q$ with $\bar{p}_{m_{i}}^{i}=0, i=1,2,3, \bar{z}^{1}<u_{2}^{1}$, $\bar{z}^{2}<u_{3}^{2}$, and $\bar{z}^{3}<u_{1}^{3}$ if and only if $\Delta_{1}^{1} \Delta_{1}^{2} \Delta_{1}^{3}<\Delta_{2}^{1} \Delta_{2}^{2} \Delta_{2}^{3}$ and $\rho$ is such that (4.76)-(4.78) are satisfied. In this case there is a unique CPE. It is given by (4.70) and (4.71). The equilibrium utilities satisfy $\bar{z}^{1}<u_{2}^{1}, \bar{z}^{2}<u_{3}^{2}$, and $\bar{z}^{3}<u_{1}^{3}$.

Notice that, unlike the equilibrium of Theorem 4.1, the option not to make a proposal cannot be chosen with any positive probability. The equilibrium is in pure strategies in which each player always proposes his best alternative and always accepts his best and middle alternative. These strategies imply immediate agreement and the probability distribution over the three alternatives coincides with the recognition probabilities, i.e. $\bar{\pi}_{i}=\rho_{i}$. The expected equilibrium utilities are below the utility of the middle alternative, which makes accepting the middle alternative the unique best response. The recognized player takes full advantage of this response by proposing his best alternative knowing it will be accepted for sure.

\subsection{Summary of Equilibrium Types}

The results obtained thus far show that many different cases and subcases have to be distinguished in characterizing all CPEs. It is astonishing that all except one of these eight (sub)cases can occur in equilibrium, albeit that three subcases are degenerate. Also by symmetry, permutations of the players' roles adds to the multiplicity of cases. Four subcases are robust in the sense of having positive Lebesgue measure in the parameter 


\begin{tabular}{|c|ccc|ccc|c|}
\hline Theorem & \multicolumn{3}{|c|}{ Proposals } & \multicolumn{3}{c|}{ Utilities } & Occurrence \\
\hline 4.1 & $\bar{p}_{2}^{1}>0$ & $\bar{p}_{3}^{2}>0$ & $\bar{p}_{1}^{3}>0$ & $\bar{z}^{1}=u_{2}^{1}$ & $\bar{z}^{2}=u_{3}^{2}$ & $\bar{z}^{3}=u_{1}^{3}$ & Degenerate \\
\hline 4.3 .1 & $\bar{p}_{2}^{1}=0$ & $\bar{p}_{3}^{2}=0$ & $\bar{p}_{1}^{3}>0$ & $\bar{z}^{1}=u_{2}^{1}$ & $\bar{z}^{2}=u_{3}^{2}$ & $\bar{z}^{3}<u_{1}^{3}$ & \\
& $\bar{p}_{2}^{1}=0$ & $\bar{p}_{3}^{2}>0$ & $\bar{p}_{1}^{3}=0$ & $\bar{z}^{1}=u_{2}^{1}$ & $\bar{z}^{2}<u_{3}^{2}$ & $\bar{z}^{3}=u_{1}^{3}$ & \\
& $\bar{p}_{2}^{1}>0$ & $\bar{p}_{3}^{2}=0$ & $\bar{p}_{1}^{3}=0$ & $\bar{z}^{1}<u_{2}^{1}$ & $\bar{z}^{2}=u_{3}^{2}$ & $\bar{z}^{3}=u_{1}^{3}$ & \\
\hline 4.3 .2 & $\bar{p}_{2}^{1}=0$ & $\bar{p}_{3}^{2}=0$ & $\bar{p}_{1}^{3}>0$ & $\bar{z}^{1}<u_{2}^{1}$ & $\bar{z}^{2}=u_{3}^{2}$ & $\bar{z}^{3}<u_{1}^{3}$ & \\
& $\bar{p}_{2}^{1}=0$ & $\bar{p}_{3}^{2}>0$ & $\bar{p}_{1}^{3}=0$ & $\bar{z}^{1}=u_{2}^{1}$ & $\bar{z}^{2}<u_{3}^{2}$ & $\bar{z}^{3}<u_{1}^{3}$ & \\
& $\bar{p}_{2}^{1}>0$ & $\bar{p}_{3}^{2}=0$ & $\bar{p}_{1}^{3}=0$ & $\bar{z}^{1}<u_{2}^{1}$ & $\bar{z}^{2}<u_{3}^{2}$ & $\bar{z}^{3}=u_{1}^{3}$ & \\
\hline 4.4 .1 & $\bar{p}_{2}^{1}=0$ & $\bar{p}_{3}^{2}=0$ & $\bar{p}_{1}^{3}=0$ & $\bar{z}^{1}=u_{2}^{1}$ & $\bar{z}^{2}=u_{3}^{2}$ & $\bar{z}^{3}=u_{1}^{3}$ & Degenerate \\
\hline 4.4 .2 & $\bar{p}_{2}^{1}=0$ & $\bar{p}_{3}^{2}=0$ & $\bar{p}_{1}^{3}=0$ & $\bar{z}^{1}=u_{2}^{1}$ & $\bar{z}^{2}=u_{3}^{2}$ & $\bar{z}^{3}<u_{1}^{3}$ & \\
& $\bar{p}_{2}^{1}=0$ & $\bar{p}_{3}^{2}=0$ & $\bar{p}_{1}^{3}=0$ & $\bar{z}^{1}=u_{2}^{1}$ & $\bar{z}^{2}<u_{3}^{2}$ & $\bar{z}^{3}=u_{1}^{3}$ & \\
& $\bar{p}_{2}^{1}=0$ & $\bar{p}_{3}^{2}=0$ & $\bar{p}_{1}^{3}=0$ & $\bar{z}^{1}<u_{2}^{1}$ & $\bar{z}^{2}=u_{3}^{2}$ & $\bar{z}^{3}=u_{1}^{3}$ & \\
\hline 4.4 .3 & $\bar{p}_{2}^{1}=0$ & $\bar{p}_{3}^{2}=0$ & $\bar{p}_{1}^{3}=0$ & $\bar{z}^{1}=u_{2}^{1}$ & $\bar{z}^{2}<u_{3}^{2}$ & $\bar{z}^{3}<u_{1}^{3}$ & Degenerate \\
& $\bar{p}_{2}^{1}=0$ & $\bar{p}_{3}^{2}=0$ & $\bar{p}_{1}^{3}=0$ & $\bar{z}^{1}<u_{2}^{1}$ & $\bar{z}^{2}=u_{3}^{2}$ & $\bar{z}^{3}<u_{1}^{3}$ & Degenerate \\
& $\bar{p}_{2}^{1}=0$ & $\bar{p}_{3}^{2}=0$ & $\bar{p}_{1}^{3}=0$ & $\bar{z}^{1}<u_{2}^{1}$ & $\bar{z}^{2}<u_{3}^{2}$ & $\bar{z}^{3}=u_{1}^{3}$ & Degenerate \\
\hline 4.4 .4 & $\bar{p}_{2}^{1}=0$ & $\bar{p}_{3}^{2}=0$ & $\bar{p}_{1}^{3}=0$ & $\bar{z}^{1}<u_{2}^{1}$ & $\bar{z}^{2}<u_{3}^{2}$ & $\bar{z}^{3}<u_{1}^{3}$ & \\
\hline
\end{tabular}

Table 2: Characteristics of the various types of equilibrium.

space. Only one of these four subcases admits a unique CPE in pure strategies. Table 2 summarizes these and other main characteristics of the equilibria as found in this section.

The robust cases have the following defining characteristics:

First, conditional on being recognized, at most one player randomizes between his best and middle alternative, and the other players always propose their best alternative for sure. To put it differently, at most one player proposes cautiously and the others aggressively.

Second, the number of players who get their best alternative accepted for sure can be any number ranging from none to three.

Third, at the start of any bargaining round during ongoing negotiations, all players can realize, in expectation, an equilibrium utility that is at most the utility of the middle alternative. It also implies that each player $i$ as a responding player faces a continuation payoff after both responding players reject equal to $\bar{z}^{i} \leq u_{m_{i}}^{i}$. In case the inequality is strict, player $i$ accepts for sure his middle alternative, whenever it is on the table. In any CPE, this will provoke player $i+1$ for whom $m_{i}$ is the best alternative to propose aggressively whenever he is recognized.

Fourth, except for some degenerate cases, the recognized player does strictly better, in expectation, than his security level that can be obtained for sure by proposing the middle alternative. This implies that each recognized player has a strict advantage in being recognized. Table 2 shows many cases in which the recognized player's best alternative is accepted for sure. It is also clear that the recognized player has an incentive to be aggressive and proposes his best alternative with positive probability, often probability equal to one.

\section{$5 \quad$ Special Cases and Examples}

In this section, we discuss two special cases of interest to illustrate the type of results obtained in the previous sections. These examples also serve as an illustration to the 
analyses that follow this section.

\subsection{The Symmetric Case}

Throughout this subsection, we will first consider a special case of interest in more detail, namely what we call the symmetric case. In the symmetric case it holds that $\rho_{1}=\rho_{2}=$ $\rho_{3}=1 / 3$, and for $i=1,2,3$, the utilities $u_{b_{i}}^{i}, u_{m_{i}}^{i}$, and $u_{w_{i}}^{i}$ are independent of $i$. Since in this section we are considering the symmetric case, we drop the superscript $i$ from $\Delta_{1}^{i}, \Delta_{2}^{i}$, and $\Delta_{3}^{i}$.

The condition of Theorem 4.1 then reduces to $\Delta_{1}=\Delta_{2}$, or equivalently, for $i=1,2,3$, $u_{1}^{i}+u_{3}^{i}=2 u_{2}^{i}$, i.e. $u_{2}^{i}$ is the average of $u_{1}^{i}$ and $u_{3}^{i}$. From our expressions for $\bar{\pi}_{j}$, we find that $\bar{\pi}_{1}=\bar{\pi}_{2}=\bar{\pi}_{3}=1 / 3$. In this case, there exists a CPE without any delay, as well as CPEs with delay. In the CPE without delay, every player $i$ proposes $x_{m_{i}}$ with probability 1 , a proposal that is accepted for sure by player $i+1$. The other CPEs are as follows. All players $i$ propose $x_{m_{i}}$ with the same probability that is strictly in between 0 and $1,0<\bar{p}_{m_{1}}^{1}=\bar{p}_{m_{2}}^{2}=\bar{p}_{m_{3}}^{3}<1$. Such a proposal is accepted for sure by player $i+1$. The remaining probability is put by player $i$ on the option not to make a proposal $x_{0}$ and the proposal $x_{b_{i}}$, for $i=1,2,3, \bar{p}_{0}^{i}+\bar{p}_{b_{i}}^{i}=1-\bar{p}_{m_{i}}^{i}$. Both proposals are identical in the sense that they do not lead to the immediate acceptance of some outcome. Equilibrium utilities satisfy $\bar{z}^{i}=u_{m_{i}}^{i}, i=1,2,3$.

Equilibria as in Theorem 4.3.1 do not exist. By (4.21) and (4.23) we have that

$$
\frac{1}{2}<\frac{\left(\Delta_{2}\right)^{3}-\left(\Delta_{1}\right)^{3}}{\left(\Delta_{1}\right)^{2} \Delta_{2}+\Delta_{1}\left(\Delta_{2}\right)^{2}+\left(\Delta_{2}\right)^{3}} \leq \frac{1}{3}
$$

a contradiction.

The conditions of Theorem 4.3.2 cannot hold. Indeed, from (4.38) it follows that $\Delta_{2}>\Delta_{1}$, whereas (4.39) implies $\Delta_{2}<\Delta_{1}$.

The condition of Theorem 4.4.1 reduces to $\Delta_{1}=\Delta_{2}$, or equivalently, for $i=1,2,3$, $u_{1}^{i}+u_{3}^{i}=2 u_{2}^{i}$, i.e. $u_{2}^{i}$ is the average of $u_{1}^{i}$ and $u_{3}^{i}$. From our expressions for $\bar{\pi}_{j}$, we find that $\bar{\pi}_{1}=\bar{\pi}_{2}=\bar{\pi}_{3}=1 / 3$. In this case, there exists a CPE without any delay, as well as CPEs with delay. In the CPE without delay, every player $i$ proposes $x_{b_{i}}$ with probability 1 , a proposal that is accepted for sure by player $i-1$. The other CPEs are as follows. All players $i$ propose $x_{b_{i}}$ with probability 1 . Such a proposal is rejected by player $i-1$ with a probability $\bar{q}_{m_{i-1}}^{i-1}$ that is independent of $i$ and strictly in between 0 and $1,0<\bar{q}_{2}^{1}=\bar{q}_{3}^{2}=\bar{q}_{1}^{3}<1$. All CPEs induce the same equilibrium utilities given by $\bar{z}^{i}=u_{m_{i}}^{i}, i=1,2,3$.

The conditions of Theorem 4.4 .2 cannot hold. Indeed, since $\left(\Delta_{1}\right)^{3}<\left(\Delta_{2}\right)^{3}$, we have that $\Delta_{1}<\Delta_{2}$. At the same time, (4.55) requires $\Delta_{2} \leq \Delta_{1}$, a contradiction.

The conditions of Theorem 4.4.3 do not hold since they require at the same time that $\Delta_{1}<\Delta_{2}$ and $\Delta_{1}=\Delta_{2}$.

The conditions of Theorem 4.4.4 hold if and only if $\Delta_{1}<\Delta_{2}$. From our expressions for $\bar{\pi}_{j}$, we find that $\bar{\pi}_{1}=\bar{\pi}_{2}=\bar{\pi}_{3}=1 / 3$. The CPE does not involve delay. Every player $i$ proposes $x_{b_{i}}$ with probability 1 , a proposal that is accepted for sure by player $i-1$. All CPEs induce the same equilibrium utilities given by $\bar{z}^{i}=\left(u_{1}^{i}+u_{2}^{i}+u_{3}^{i}\right) / 3<u_{m_{i}}^{i}, i=1,2,3$.

Summarizing, we have the following result. 
ThEOREM 5.1 For $i=1,2,3$, let $u_{b_{i}}^{i}, u_{m_{i}}^{i}, u_{w_{i}}^{i}$, and $\rho_{i}$ be independent of $i$. There is a CPE $(\bar{p}, \bar{q}) \in P \times Q$ if and only if $\Delta_{1} \leq \Delta_{2}$. There is a CPE with the property that $\bar{p}_{b_{i}}^{i}=1$ and $\bar{q}_{m_{i}}^{i}=0, i=1,2,3$. When $\Delta_{1}<\Delta_{2}$ this is the unique CPE. When $\Delta_{1}=\Delta_{2}$, there are also equilibria $(\bar{p}, \bar{q}) \in P \times Q$ with $\bar{p}_{b_{i}}^{i}=1$ and $\bar{q}_{m_{i}}^{i}>0, i=1,2,3$, and equilibria $(\bar{p}, \bar{q}) \in P \times Q$ with $\bar{p}_{m_{i}}^{i}>0$ and $\bar{q}_{m_{i}}^{i}=1, i=1,2$, 3. In the last type of equilibrium, some players may actually use the option not to make a proposal with strictly positive probability less than 1. Equilibrium utilities satisfy $z^{i}=\left(u_{1}^{i}+u_{2}^{i}+u_{3}^{i}\right) / 3, i=1,2,3$ in all cases.

The equilibria in Theorem 5.1 are all symmetric in the sense that either all players $i$ propose $x_{b_{i}}$ with equal probability, which is accepted with strictly positive probability independent from $i$, or all players $i$ propose $x_{m_{i}}$ with equal probability, which is accepted with strictly positive probability independent from $i$, whereas a proposal $x_{b_{i}}$ is rejected for sure. The latter situation may only occur in the degenerate case where $\Delta_{1}=\Delta_{2}$. In this degenerate case, there is a modest degree of asymmetry possible: some players may decide not to make a proposal with probability strictly less than one, potentially dependent on the identity of the player.

CPEs are unique when $\Delta_{1}<\Delta_{2}$. They are essentially unique when $\Delta_{1}=\Delta_{2}$ in the sense that in the latter case all equilibria share the same realization probabilities $\bar{\pi}_{1}=\bar{\pi}_{2}=$ $\bar{\pi}_{3}=1 / 3$ of the possible outcomes, and therefore share the same equilibrium utilities. It follows that equilibrium utilities are given by $z^{i}=\left(u_{1}^{i}+u_{2}^{i}+u_{3}^{i}\right) / 3, i=1,2,3$.

\subsection{The Asymmetric Case: An Example}

To study the asymmetric case, we consider the following example that allows for asymmetric recognition probabilities in more detail. In this subsection we assume that

$$
\begin{aligned}
& \Delta_{1}^{1}=\Delta_{1}^{2}=\Delta_{1}^{3}=1, \\
& \Delta_{2}^{1}=\Delta_{2}^{2}=\Delta_{2}^{3}=2,
\end{aligned}
$$

and an arbitrary $\rho$ is taken. We normalize the utility of the worst alternative of a player to 0 .

Equilibria as in Theorem 4.1 and 4.4.1 do not exist.

The conditions of Theorem 4.3.1 and its symmetric analogues reduce to

$$
\begin{aligned}
& \rho_{1}<\rho_{3} \text { and } \rho_{2} \geq \frac{1}{2}, \\
& \rho_{2}<\rho_{1} \text { and } \rho_{3} \geq \frac{1}{2}, \\
& \rho_{3}<\rho_{2} \text { and } \rho_{1} \geq \frac{1}{2} .
\end{aligned}
$$

The conditions of Theorem 4.3.2 and its symmetric analogues reduce to

$$
\begin{aligned}
& 2 \rho_{1}<\rho_{2} \text { and } \rho_{2}<\frac{1}{2}, \\
& 2 \rho_{2}<\rho_{3} \text { and } \rho_{3}<\frac{1}{2}, \\
& 2 \rho_{3}<\rho_{1} \text { and } \rho_{1}<\frac{1}{2} .
\end{aligned}
$$

The conditions of Theorem 4.4.2 and its symmetric analogues reduce to

$$
\begin{aligned}
\rho_{3} & \leq \rho_{1} \leq 2 \rho_{3} \text { and } 2 \rho_{1} \leq \rho_{2}, \\
\rho_{1} & \leq \rho_{2} \leq 2 \rho_{1} \text { and } 2 \rho_{2} \leq \rho_{3}, \\
\rho_{2} & \leq \rho_{3} \leq 2 \rho_{2} \text { and } 2 \rho_{3} \leq \rho_{1} .
\end{aligned}
$$


The conditions of Theorem 4.4.3 and its symmetric analogues reduce to

$$
\begin{aligned}
& \rho_{1}<\frac{4}{7} \text { and } 2 \rho_{3}=\rho_{1}, \\
& \rho_{2}<\frac{4}{7} \text { and } 2 \rho_{1}=\rho_{2}, \\
& \rho_{3}<\frac{4}{7} \text { and } 2 \rho_{2}=\rho_{3} .
\end{aligned}
$$

The conditions of Theorem 4.4.4 and its symmetric analogues reduce to

$$
\begin{aligned}
\rho_{1} & <2 \rho_{3}, \\
\rho_{2} & <2 \rho_{1}, \\
\rho_{3} & <2 \rho_{2} .
\end{aligned}
$$

We illustrate the above in the following figure. The areas corresponding to Theo-

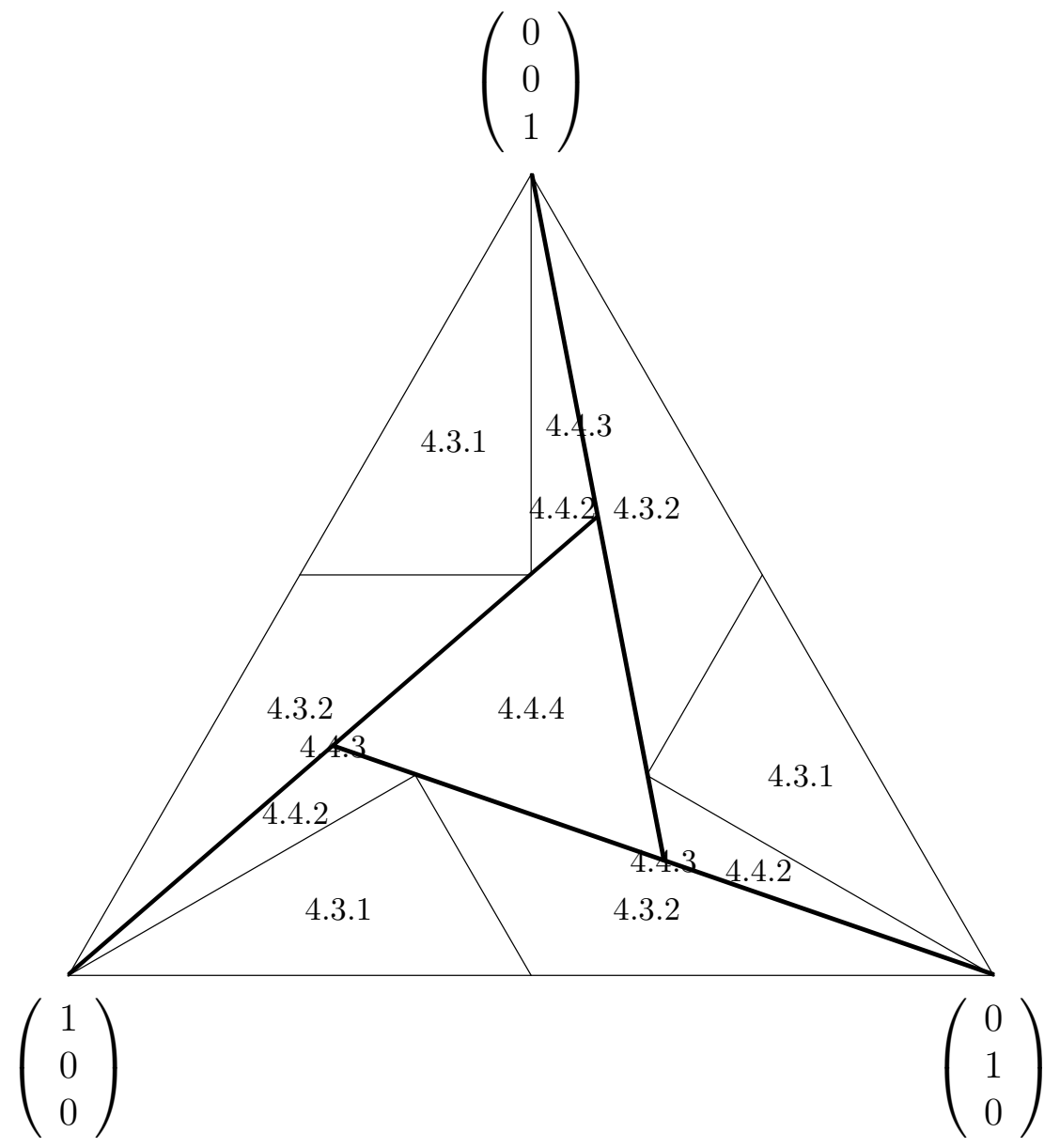

Figure 1: Conditions on $\rho$ for which particular types of equilibria exist.

rem 4.3.2 and 4.4.4 are open, and the areas corresponding to Theorem 4.4.2 are closed. The areas corresponding to Theorem 4.3 .1 are closed when adjacent to the area of Theorem 4.3.2 and open when adjacent to Theorem 4.4.2. The area corresponding to Theorem 4.4.3 is drawn by thick lines. The theorems almost partition the unit simplex, with 
the exception of the intersection of the areas corresponding to Theorem 4.4.2 and 4.4.3, where different types of equilibria overlap.

The next figure indicates the utility level of player 1 per area. Player 1's equilibrium utility is not monotonic in his recognition probability. The intuition is that next to player 1's own recognition probability, the recognition probability of player 3 matters. When player 3 has a high recognition probability, then he will frequently propose the third outcome, which is the worst case scenario from the perspective of player 1 . The correspondence associating to each $\rho$ the set of possible equilibrium utility levels of player 1 is upper hemi-continuous, but not continuous. It is multiple valued in the area corresponding to Theorem 4.4.3.

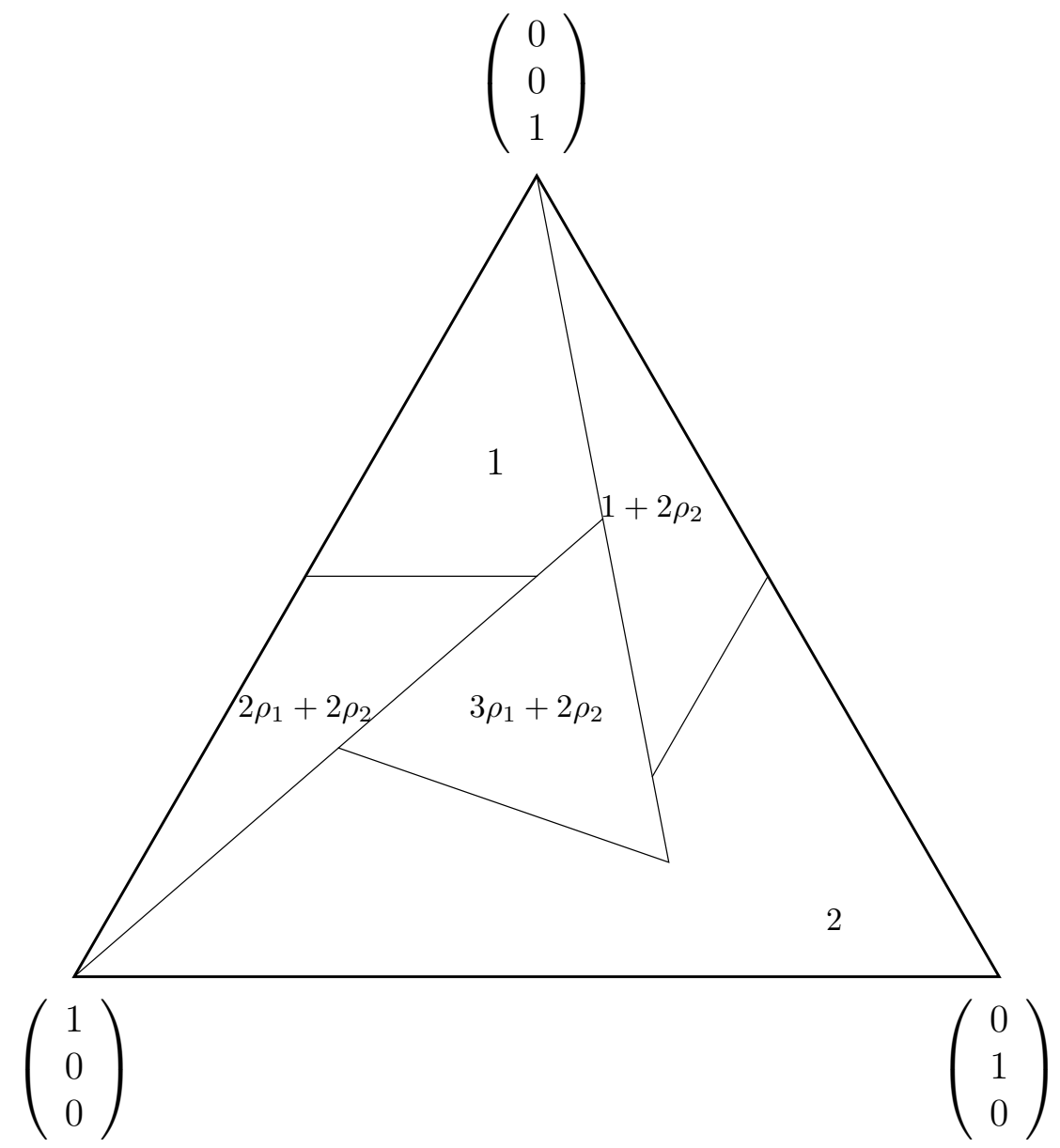

Figure 2: Equilibrium utility of player 1 in relation to $\rho$.

The next figure depicts the probability of delay. Notice that if this probability is equal to $\alpha$, then the expected delay is $\alpha /(1-\alpha)$ periods. Delay varies in an upper hemi-continuous way with recognition probabilities $\rho$. The probability of delay is zero when recognition probabilities are all close to $1 / 3$. When one or two recognition probabilities converge to zero, then the probability of delay converges to one, so the expected delay converges to infinity. 


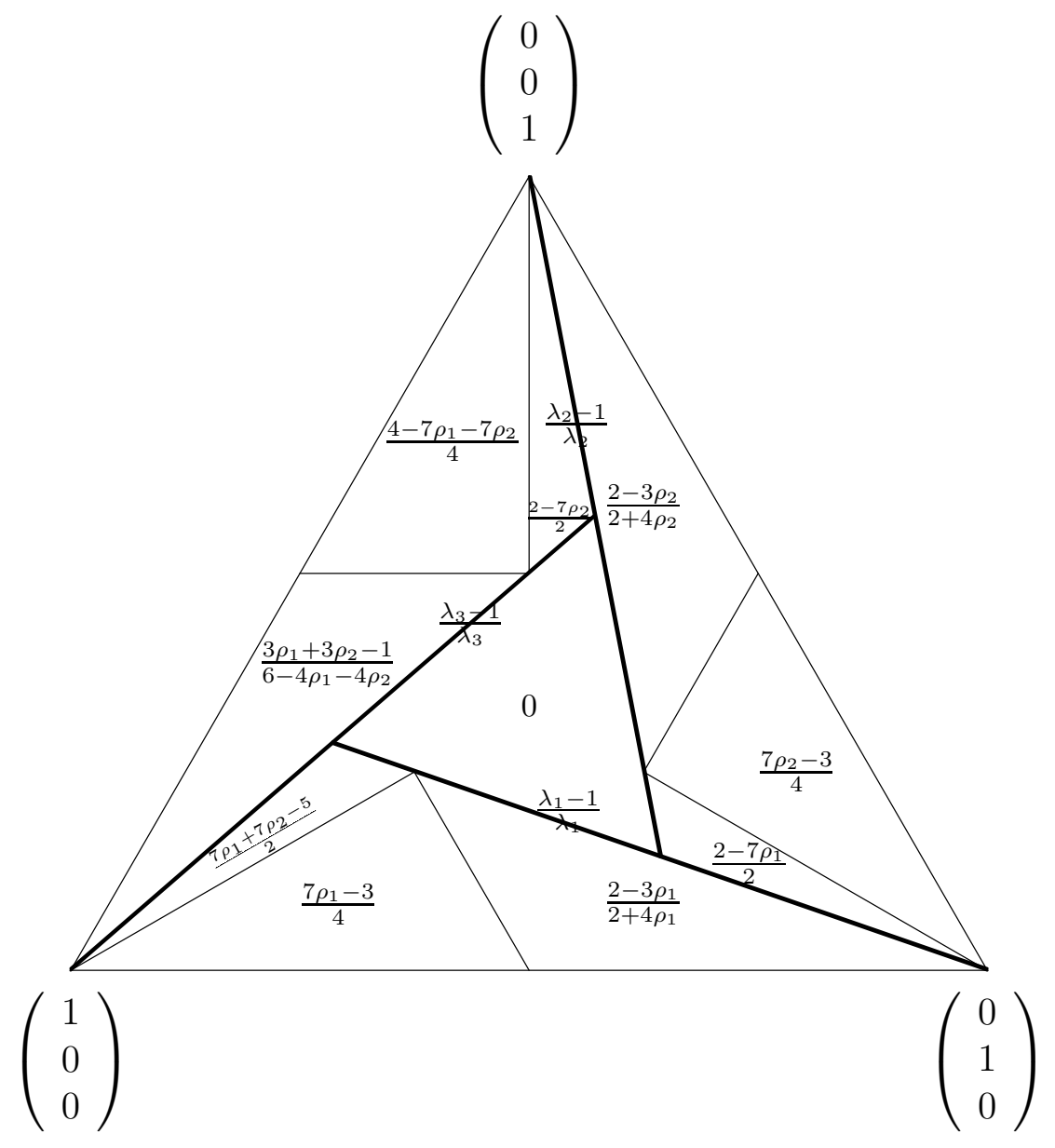

Figure 3: The probability of delay in relation to $\rho$. For region 4.4 .3 it holds that $\lambda_{1} \in$ $\left(\frac{2}{7 \rho_{1}}, \frac{4}{7 \rho_{1}}\right) \cap\left[1, \frac{2+4 \rho_{1}}{7 \rho_{1}}\right], \lambda_{2} \in\left(\frac{2}{7 \rho_{2}}, \frac{4}{7 \rho_{2}}\right) \cap\left[1, \frac{2+4 \rho_{2}}{7 \rho_{2}}\right]$, and $\lambda_{3} \in\left(\frac{2}{7-7 \rho_{1}-7 \rho_{2}}, \frac{4}{7-7 \rho_{1}-7 \rho_{2}}\right) \cap\left[1, \frac{6-4 \rho_{1}-4 \rho_{2}}{7-7 \rho_{1}-7 \rho_{2}}\right]$. 


\section{Existence and Uniqueness of Equilibria}

The results of Section 4 establish that equilibria can only exist if $\Delta_{1}^{1} \Delta_{1}^{2} \Delta_{1}^{3} \leq \Delta_{2}^{1} \Delta_{2}^{2} \Delta_{2}^{3}$. In this section we show that this condition is not only necessary, but also sufficient for the existence of equilibria. Both Theorem 4.1 and Theorem 4.4.1 imply that equilibria exist for all values of the recognition probabilities $\rho$ when $\Delta_{1}^{1} \Delta_{1}^{2} \Delta_{1}^{3}=\Delta_{2}^{1} \Delta_{2}^{2} \Delta_{2}^{3}$. Therefore we only have to examine whether equilibria exist when $\Delta_{1}^{1} \Delta_{1}^{2} \Delta_{1}^{3}<\Delta_{2}^{1} \Delta_{2}^{2} \Delta_{2}^{3}$, irrespective of the values of the recognition probabilities $\rho$.

It is instructive to define the risk coefficient $\alpha_{i}$ of player $i$ by

$$
\alpha_{i}=\frac{\Delta_{1}^{i}}{\Delta_{2}^{i}}, \quad i=1,2,3
$$

The risk coefficient is closely related to the concept of risk limit as introduced in Zeuthen (1930) and further developed in Harsanyi (1977). The risk limit is defined in a setting with two players and three outcomes. There is the outcome proposed by the player himself, say $y_{1}$, the outcome proposed by his opponent, say $y_{2}$, and the disagreement outcome, say $y_{0}$. The risk limit of a player is then defined as the probability on the disagreement outcome for which he would be indifferent between getting the disagreement outcome with this probability and $y_{1}$ with the remaining probability, and getting outcome $y_{2}$ for sure. In a formula the risk limit $\ell$ is given by

$$
\ell=\frac{u\left(y_{1}\right)-u\left(y_{2}\right)}{u\left(y_{1}\right)-u\left(y_{0}\right)}
$$

This paper involves three players and four alternatives (we now count the disagreement outcome as one alternative), so the risk limit is not directly applicable. However, if we define $y_{1}$ as the best alternative $x_{b_{i}}$ for player $i, y_{2}$ as his middle alternative $x_{m_{i}}$, and $y_{0}$ as his worst alternative $x_{w_{i}}$, then a straightforward calculation reveals that

$$
\ell_{i}=\frac{\Delta_{1}^{i}}{\Delta_{1}^{i}+\Delta_{2}^{i}}=\frac{\alpha_{i}}{1+\alpha_{i}}
$$

Alternatively, we can write $\alpha_{i}=\ell_{i} /\left(1-\ell_{i}\right)$.

The condition $\Delta_{1}^{1} \Delta_{1}^{2} \Delta_{1}^{3} \leq \Delta_{2}^{1} \Delta_{2}^{2} \Delta_{2}^{3}$ can be rewritten as $\alpha_{1} \alpha_{2} \alpha_{3} \leq 1$, or as $\sqrt[3]{\alpha_{1} \alpha_{2} \alpha_{3}} \leq 1$. In words this condition expresses that the geometric mean of the players' risk coefficients is less than or equal to 1.

To simplify the analysis, we introduce some additional notation, in particular the coefficients $\beta_{i}$, defined by

$$
\begin{aligned}
\beta_{1} & =\frac{\Delta_{2}^{1} \Delta_{2}^{2} \Delta_{2}^{3}-\Delta_{1}^{1} \Delta_{1}^{2} \Delta_{1}^{3}}{\Delta_{1}^{1} \Delta_{1}^{2} \Delta_{1}^{3}+\Delta_{1}^{1} \Delta_{2}^{2} \Delta_{1}^{3}+\Delta_{1}^{1} \Delta_{2}^{2} \Delta_{2}^{3}}, \\
\beta_{2} & =\frac{\Delta_{2}^{1} \Delta_{2}^{2} \Delta_{2}^{3}-\Delta_{1}^{1} \Delta_{1}^{2} \Delta_{1}^{3}}{\Delta_{1}^{1} \Delta_{1}^{2} \Delta_{1}^{3}+\Delta_{1}^{1} \Delta_{1}^{2} \Delta_{2}^{3}+\Delta_{2}^{1} \Delta_{1}^{2} \Delta_{2}^{3}}, \\
\beta_{3} & =\frac{\Delta_{2}^{1} \Delta_{2}^{2} \Delta_{2}^{3}-\Delta_{1}^{1} \Delta_{1}^{2} \Delta_{1}^{3}}{\Delta_{1}^{1} \Delta_{1}^{2} \Delta_{1}^{3}+\Delta_{2}^{1} \Delta_{1}^{2} \Delta_{1}^{3}+\Delta_{2}^{1} \Delta_{2}^{2} \Delta_{1}^{3}} .
\end{aligned}
$$




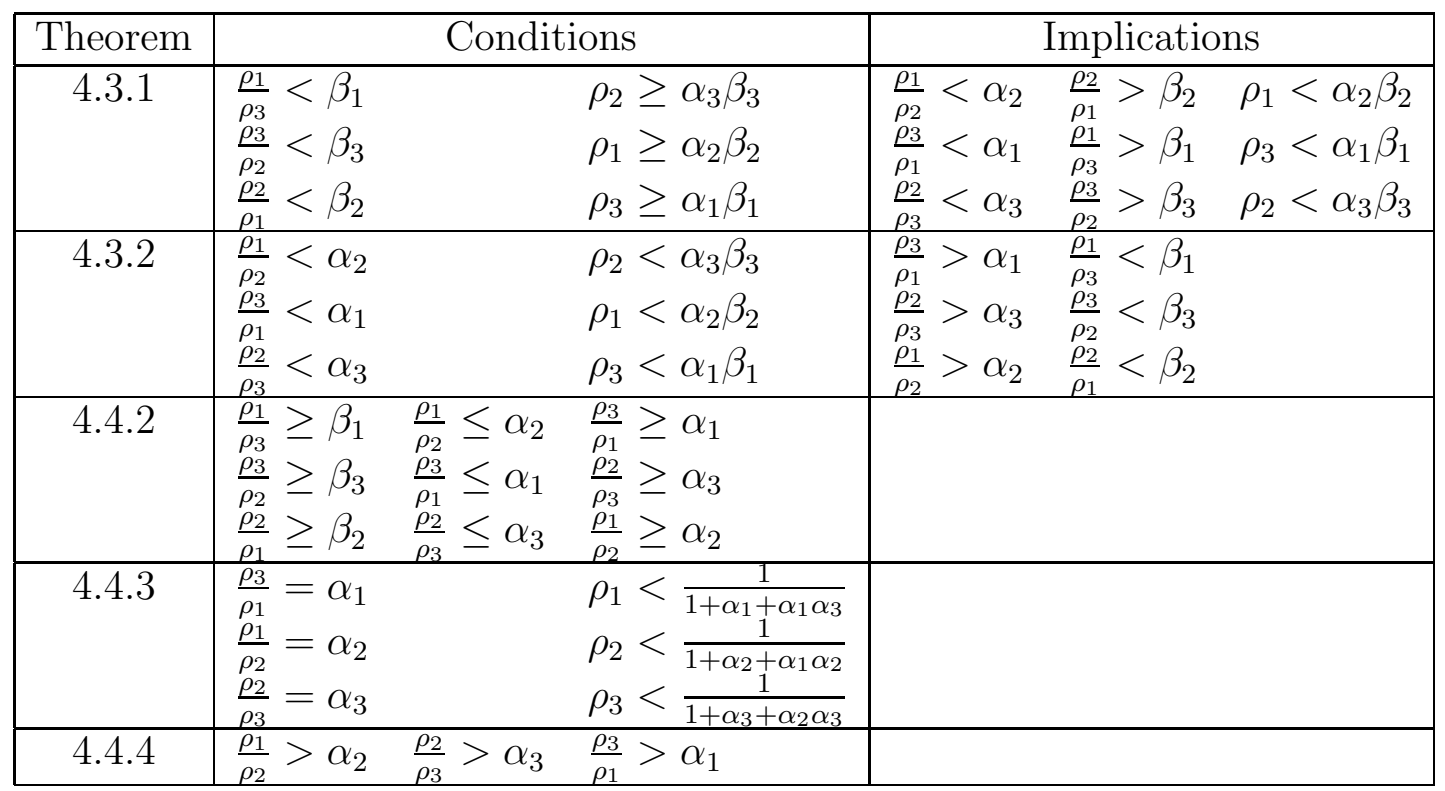

Table 3: Equilibrium types and conditions on recognition probabilities.

In the definition of $\beta_{i}$ it holds that $\Delta_{1}^{i}$ enters in each term in the denominator. To manipulate the conditions as expressed in $\alpha_{i}$ and $\beta_{i}$, it is useful to note that

$$
\begin{aligned}
& \frac{\beta_{1}}{1+\beta_{1}}=\alpha_{2} \beta_{2}, \quad \beta_{1}=\frac{1-\alpha_{1} \alpha_{2} \alpha_{3}}{\alpha_{1} \alpha_{2} \alpha_{3}+\alpha_{1} \alpha_{3}+\alpha_{1}}, \\
& \frac{\beta_{2}}{1+\beta_{2}}=\alpha_{3} \beta_{3}, \quad \beta_{2}=\frac{1-\alpha_{1} \alpha_{2} \alpha_{3}}{\alpha_{1} \alpha_{2} \alpha_{3}+\alpha_{1} \alpha_{2}+\alpha_{2}}, \\
& \frac{\beta_{3}}{1+\beta_{3}}=\alpha_{1} \beta_{1}, \quad \beta_{3}=\frac{1-\alpha_{1} \alpha_{2} \alpha_{3}}{\alpha_{1} \alpha_{2} \alpha_{3}+\alpha_{2} \alpha_{3}+\alpha_{3}} .
\end{aligned}
$$

The conditions on recognition probabilities identified in Section 4 under which equilibria of a particular type exist are summarized in Table 3. Subtypes are ordered in the same way as in Table 2. For all equilibrium types listed in Table 3 it holds that $\Delta_{1}^{1} \Delta_{1}^{2} \Delta_{1}^{3}<\Delta_{2}^{1} \Delta_{2}^{2} \Delta_{2}^{3}$, or equivalently, $\alpha_{1} \alpha_{2} \alpha_{3}<1$. Table 3 also shows that the conditions for which particular types of equilibria exist can be formulated in terms of the players' risk coefficients (since also $\beta_{i}$ can be expressed in terms of $\alpha_{1}, \alpha_{2}$, and $\alpha_{3}$ ) and recognition probability vector $\rho$ only.

The last column of Table 3 states some of the implications that follow from the conditions on the parameters. We show next how these implications can be derived.

Consider the first line of conditions in Theorem 4.3.1 and suppose by way of contradiction that $\rho_{1} / \rho_{2} \geq \alpha_{2}$. Then

$$
1=\rho_{1}+\rho_{2}+\rho_{3}>\alpha_{2} \alpha_{3} \beta_{3}+\alpha_{3} \beta_{3}+\frac{\alpha_{2} \alpha_{3} \beta_{3}}{\beta_{1}}=1,
$$

a contradiction, so consequently $\rho_{1} / \rho_{2}<\alpha_{2}$. Similarly, the supposition $\rho_{2} / \rho_{1} \leq \beta_{2}$ implies

$$
1=\rho_{1}+\rho_{2}+\rho_{3}>\frac{\alpha_{3} \beta_{3}}{\beta_{2}}+\alpha_{3} \beta_{3}+\frac{\alpha_{3} \beta_{3}}{\beta_{1} \beta_{2}}=1+\frac{\alpha_{3} \beta_{3}}{\beta_{1} \beta_{2}}>1,
$$

a contradiction, so consequently $\rho_{2} / \rho_{1}>\beta_{2}$. Also, the supposition $\rho_{1} \geq \alpha_{2} \beta_{2}$ implies

$$
1=\rho_{1}+\rho_{2}+\rho_{3}>\alpha_{2} \beta_{2}+\alpha_{3} \beta_{3}+\frac{\alpha_{2} \beta_{2}}{\beta_{1}}=1+\alpha_{3} \beta_{3}>1
$$


a contradiction, so consequently $\rho_{1}<\alpha_{2} \beta_{2}$. The other implications of Theorem 4.3 .1 follow by symmetry.

Consider the first line of conditions in Theorem 4.3.2 and suppose by way of contradiction $\rho_{3} / \rho_{1} \leq \alpha_{1}$. Then we obtain

$$
1=\rho_{1}+\rho_{2}+\rho_{3}<\alpha_{2} \alpha_{3} \beta_{3}+\alpha_{3} \beta_{3}+\alpha_{1} \alpha_{2} \alpha_{3} \beta_{3}=\left(1-\alpha_{1} \alpha_{2} \alpha_{3}\right)<1,
$$

a contradiction, so consequently $\rho_{3} / \rho_{1}>\alpha_{1}$. Similarly, the supposition $\rho_{1} / \rho_{3} \geq \beta_{1}$ implies

$$
1=\rho_{1}+\rho_{2}+\rho_{3}<\alpha_{2} \alpha_{3} \beta_{3}+\alpha_{3} \beta_{3}+\frac{\alpha_{2} \alpha_{3} \beta_{3}}{\beta_{1}}=1
$$

a contradiction, so consequently $\rho_{1} / \rho_{3}<\beta_{1}$. The other implications of Theorem 4.3.2 follow by symmetry.

THEOREM 6.1 There exists a CPE if and only if the geometric mean of the players' risk coefficients is less than or equal to 1.

PROOF: All results in Section 4 impose the condition $\Delta_{1}^{1} \Delta_{1}^{2} \Delta_{1}^{3} \leq \Delta_{2}^{1} \Delta_{2}^{2} \Delta_{2}^{3}$ for equilibrium existence. By Theorem 4.1 an equilibrium exists if $\Delta_{1}^{1} \Delta_{1}^{2} \Delta_{1}^{3}=\Delta_{2}^{1} \Delta_{2}^{2} \Delta_{2}^{3}$. It remains to be shown that an equilibrium exists if $\Delta_{1}^{1} \Delta_{1}^{2} \Delta_{1}^{3}<\Delta_{2}^{1} \Delta_{2}^{2} \Delta_{2}^{3}$.

By Theorem 4.4.4 an equilibrium exists if $\rho_{1} / \rho_{2}>\alpha_{2}, \rho_{2} / \rho_{3}>\alpha_{3}$, and $\rho_{3} / \rho_{1}>\alpha_{1}$. Consider now the cases where the conditions of Theorem 4.4.4 are not satisfied. We claim that then

$$
\left(\frac{\rho_{3}}{\rho_{1}} \geq \alpha_{1} \text { and } \frac{\rho_{1}}{\rho_{2}} \leq \alpha_{2}\right) \text { or }\left(\frac{\rho_{1}}{\rho_{2}} \geq \alpha_{2} \text { and } \frac{\rho_{2}}{\rho_{3}} \leq \alpha_{3}\right) \text { or }\left(\frac{\rho_{2}}{\rho_{3}} \geq \alpha_{3} \text { and } \frac{\rho_{3}}{\rho_{1}} \leq \alpha_{1}\right) .
$$

Indeed, assume, without loss of generality, $\rho_{1} / \rho_{2} \leq \alpha_{2}$. Either it holds that $\rho_{3} / \rho_{1} \geq \alpha_{1}$ or $\rho_{3} / \rho_{1}<\alpha_{1}$. In the former case the first formula in (6.1) is true, in the latter case it should hold that $\rho_{2} / \rho_{3} \geq \alpha_{3}$, since otherwise

$$
1=\frac{\rho_{1}}{\rho_{2}} \frac{\rho_{2}}{\rho_{3}} \frac{\rho_{3}}{\rho_{1}}<\alpha_{2} \alpha_{3} \alpha_{1}<1
$$

and the third formula in (6.1) is true.

We show next that an equilibrium exists whenever $\rho_{3} / \rho_{1} \geq \alpha_{1}$ and $\rho_{1} / \rho_{2} \leq \alpha_{2}$. The other two cases in (6.1) follow by symmetry. If $\rho_{3} / \rho_{1}=\alpha_{1}$ and $\rho_{1} / \rho_{2} \leq \alpha_{2}$, then line 1 in Theorem 4.4.3 implies the existence of an equilibrium since $\rho_{1} / \rho_{2} \leq \alpha_{2}$ implies $\rho_{1}<$ $1 /\left(1+\alpha_{1}+\alpha_{1} \alpha_{3}\right)$. Suppose, by contradiction, that $\rho_{1} \geq 1 /\left(1+\alpha_{1}+\alpha_{1} \alpha_{3}\right)$. Then

$$
\begin{aligned}
1 & =\rho_{1}+\rho_{2}+\rho_{3} \geq \frac{1}{1+\alpha_{1}+\alpha_{1} \alpha_{3}}+\frac{1}{\alpha_{2}+\alpha_{1} \alpha_{2}+\alpha_{1} \alpha_{2} \alpha_{3}}+\frac{\alpha_{1}}{1+\alpha_{1}+\alpha_{1} \alpha_{3}} \\
& =\frac{1+\alpha_{2}+\alpha_{1} \alpha_{2}}{\alpha_{2}+\alpha_{1} \alpha_{2}+\alpha_{1} \alpha_{2} \alpha_{3}}>1,
\end{aligned}
$$

a contradiction. If $\rho_{1} / \rho_{2}=\alpha_{2}$ and $\rho_{3} / \rho_{1}>\alpha_{1}$, then line 2 in Theorem 4.4 .3 implies the existence of an equilibrium since $\rho_{3} / \rho_{1} \geq \alpha_{1}$ implies $\rho_{2}<1 /\left(1+\alpha_{2}+\alpha_{1} \alpha_{2}\right)$. Suppose, by contradiction, that $\rho_{2} \geq 1 /\left(1+\alpha_{2}+\alpha_{1} \alpha_{2}\right)$. Then

$$
1=\rho_{1}+\rho_{2}+\rho_{3}>\frac{\alpha_{2}}{1+\alpha_{2}+\alpha_{1} \alpha_{2}}+\frac{1}{1+\alpha_{2}+\alpha_{1} \alpha_{2}}+\frac{\alpha_{1} \alpha_{2}}{1+\alpha_{2}+\alpha_{1} \alpha_{2}}=1,
$$


a contradiction.

It remains to be shown that an equilibrium exists if $\rho_{3} / \rho_{1}>\alpha_{1}$ and $\rho_{1} / \rho_{2}<\alpha_{2}$. By line 1 in Theorem 4.4.2, an equilibrium exists if $\rho_{3} / \rho_{1} \geq \alpha_{1}, \rho_{1} / \rho_{2} \leq \alpha_{2}$, and $\rho_{1} / \rho_{3} \geq \beta_{1}$, and by line 1 in Theorem 4.3.1 an equilibrium exists if $\rho_{1} / \rho_{3}<\beta_{1}$ and $\rho_{2} \geq \alpha_{3} \beta_{3}$.

It remains to be shown that an equilibrium exists if $\rho_{3} / \rho_{1}>\alpha_{1}, \rho_{1} / \rho_{2}<\alpha_{2}, \rho_{1} / \rho_{3}<\beta_{1}$, and $\rho_{2}<\alpha_{3} \beta_{3}$. This follows from line 1 in Theorem 4.3.2.

Q.E.D.

The necessary and sufficient condition for existence allows for one or two risk coefficients that are larger than one, but then at least one player's risk coefficient should be sufficiently below one. A risk coefficient bounded by one has a nice economic interpretation, related to the following definition.

DEFINITION 6.2 The utility function $u^{i}$ is compromise-prone if

$$
u^{i}\left(x_{m_{i}}\right) \geq \frac{1}{2} u_{i}\left(x_{b_{i}}\right)+\frac{1}{2} u_{i}\left(x_{w_{i}}\right) .
$$

A utility function is said to be compromise-prone if the utility of the middle alternative is at least as high as getting the best alternative with probability one half and getting the worst alternative with probability one half. This condition has in our discrete setting the same interpretation as risk-aversion (concavity) for an environment with a continuum of alternatives. The opposite, a lack of compromise proneness, implies risk-seeking (convexity). A player $i$ has a compromise-prone utility function if and only if $\alpha_{i} \leq 1$. The following corollary now follows immediately.

COROLlARY 6.3 If all players have compromise-prone utility functions, then there exists a CPE.

From Table 3, it immediately follows that the existence of pure CPEs with immediate agreement for symmetric bargaining protocols, i.e. $\rho_{1}=\rho_{2}=\rho_{3}=\frac{1}{3}$, is equivalent to having three compromise-prone players. So, for symmetric bargaining protocols, compromiseprone players are necessary and sufficient for existence of a pure CPE with immediate agreement in which recognized players propose their best alternative for sure and each player accepts his best and middle alternative for sure.

In general, existence of CPEs requires that at least some player should be sufficiently compromise-prone to compensate for the possible lack of such proneness of the other players. Compromise-prone players have an incentive to seek agreement.

The examples in Section 5 show that equilibrium may not be unique and that there might be infinitely many equilibrium utilities. The following result demonstrates that such examples are degenerate in the sense that this set of games has a closure with Lebesgue measure zero. To compute the Lebesgue measure of a set of games, we consider a game $(u, \rho)$ as a subset of $\mathbb{R}^{9} \times \mathbb{R}^{2}$, i.e. identifying $\rho$ by its first two coordinates. To require the zero Lebesgue measure property for the closure of a set of games, evidently implies this property for the set of games itself, but not vice versa, as for instance illustrated by the set of rational numbers. 


\begin{tabular}{|c|c|c|}
\hline Theorem & Delay probability & Occurrence \\
\hline 4.1 & {$\left[1-\left(1+\alpha_{2}+\alpha_{1} \alpha_{2}\right) \min \left\{\rho_{1}, \frac{\rho_{2}}{\alpha_{1} \alpha_{2}}, \frac{\rho_{3}}{\alpha_{2}}\right\}, 1\right)$} & Degenerate \\
\hline 4.3 .1 & $\begin{array}{l}\rho_{2}+\left(\alpha_{1} \alpha_{2} \alpha_{3}\left(1+\beta_{2}\right)-\beta_{2}\right)\left(1-\rho_{2}\right) \\
\rho_{1}+\left(\alpha_{1} \alpha_{2} \alpha_{3}\left(1+\beta_{1}\right)-\beta_{1}\right)\left(1-\rho_{1}\right) \\
\rho_{3}+\left(\alpha_{1} \alpha_{2} \alpha_{3}\left(1+\beta_{3}\right)-\beta_{3}\right)\left(1-\rho_{3}\right)\end{array}$ & \\
\hline 4.3 .2 & $\begin{array}{l}\frac{\alpha_{3}-\left(1+\alpha_{2}\right) \alpha_{3} \rho_{2}}{\left.\alpha_{3}+\rho_{2}\right)} \\
\frac{\alpha_{2}-\left(1+\alpha_{1}\right) \alpha_{2} \rho_{1}}{\alpha_{2}+\rho_{1}} \\
\frac{\alpha_{1}-\left(1+\alpha_{3}\right) \alpha_{1} \rho_{3}}{\alpha_{1}+\rho_{3}} \\
\end{array}$ & \\
\hline 4.4 .1 & $1-\left(1+\alpha_{2}+\alpha_{1} \alpha_{2}\right) \min \left\{\frac{\rho_{1}}{\alpha_{2}}, \rho_{2}, \frac{\rho_{3}}{\alpha_{1} \alpha_{2}}\right\}$ & Degenerate \\
\hline 4.4 .2 & $\begin{array}{l}1-\frac{1+\alpha_{2}+\alpha_{1} \alpha_{2}}{\alpha_{2}} \rho_{1} \\
1-\frac{1+\alpha_{1}+\alpha_{1} \alpha_{3}}{\alpha_{1}} \rho_{3} \\
1-\frac{1+\alpha_{3}+\alpha_{2} \alpha_{3}}{\alpha_{3}} \rho_{2}\end{array}$ & \\
\hline 4.4 .3 & $\begin{array}{l}D_{1} \\
D_{2} \\
D_{3}\end{array}$ & $\begin{array}{l}\text { Degenerate } \\
\text { Degenerate } \\
\text { Degenerate }\end{array}$ \\
\hline 4.4 .4 & 0 & \\
\hline $\begin{aligned} D_{1} & =[\mathrm{ma} \\
D_{2} & =[\mathrm{ma} \\
D_{3} & =[\mathrm{ma}\end{aligned}$ & $\begin{array}{l}\left.\left\{0,1-\frac{1+\alpha_{2}+\alpha_{1} \alpha_{2} \rho_{1}}{\alpha_{2}}\right\}, 1-\frac{1+\alpha_{2}+\alpha_{1} \alpha_{2}}{\alpha_{2}+\rho_{1}} \rho_{1}\right] \cap[\mathrm{m} \\
\left.\left\{0,1-\frac{1+\alpha_{3}+\alpha_{2} \alpha_{3} \rho_{2}}{\alpha_{3}}\right\}, 1-\frac{1+\alpha_{3}+\alpha_{2} \alpha_{3}}{\alpha_{3}+\rho_{2}} \rho_{2}\right] \cap[\mathrm{m} \\
\left.\left\{0,1-\frac{1+\alpha_{1}+\alpha_{1} \alpha_{3} \rho_{3}}{\alpha_{1}}\right\}, 1-\frac{1+\alpha_{1}+\alpha_{1} \alpha_{3}}{\alpha_{1}+\rho_{3}} \rho_{3}\right] \cap[\mathrm{m}\end{array}$ & $\begin{array}{l}\left.\operatorname{ax}\left\{0,1-\frac{1+\alpha_{2}+\alpha_{1} \alpha_{2} \rho_{1}}{\alpha_{2}}\right\}, 1-\left(1+\alpha_{1}+\alpha_{1} \alpha_{3}\right) \rho_{1}\right) \\
\left.\operatorname{ax}\left\{0,1-\frac{1+\alpha_{3}+\alpha_{2} \alpha_{3} \rho_{2}}{\alpha_{3}}\right\}, 1-\left(1+\alpha_{2}+\alpha_{1} \alpha_{2}\right) \rho_{2}\right) \\
\left.\operatorname{ax}\left\{0,1-\frac{1+\alpha_{1}+\alpha_{1} \alpha_{3} \rho_{3}}{\alpha_{1}}\right\}, 1-\left(1+\alpha_{3}+\alpha_{2} \alpha_{3}\right) \rho_{3}\right)\end{array}$ \\
\hline
\end{tabular}

Table 4: Delay probabilities.

TheOREM 6.4 Consider the set of games $(u, \rho) \in \mathcal{G}$ where the geometric mean of the players' risk coefficients is less than or equal to 1. Except for a subset of games whose closure has Lebesgue measure zero, there is a unique CPE.

Proof: Existence follows from Theorem 6.1. Leave out the games satisfying the conditions of Theorem 4.3.1, Theorem 4.1, and Theorem 4.4.1. This corresponds to a set of games whose closure has Lebesgue measure zero. Comparing the conditions in any two distinct rows (that do not correspond to Theorem 4.3.1) of Table 3 leads to the conclusion that the corresponding two sets of parameters have an empty intersection. This conclusion follows directly in most cases. In some cases one has to make use of the property that $\alpha_{i} \beta_{i}<1$, which implies that we cannot have simultaneously $\rho_{j} / \rho_{k}<\alpha_{i}$ and $\rho_{k} / \rho_{j}<\beta_{i}$.

\section{$7 \quad$ Delay and Cycles}

We analyze the extent to which there can be delay in the CPE. If the probability that there is delay is $\alpha$, then the expected delay is equal to $\alpha /(1-\alpha)$ periods. Using the results of Section 4 , it is a straightforward exercise to compute the probability of delay. Table 4 gives an overview of the delay probabilities. In some cases the delay probability is given by an interval. This means that for every value of delay in the interval, there is an equilibrium with that probability of delay.

An important question is whether there always exist some $\rho$ that implies existence of a CPE without delay. 
THEOREM 7.1 Let u be such that the geometric mean of the players' risk coefficients is less than or equal to one. Then there is a $\rho$ such that $(u, \rho)$ has a CPE without delay.

Proof: First we consider the case where $\Delta_{1}^{1} \Delta_{1}^{2} \Delta_{1}^{3}=\Delta_{2}^{1} \Delta_{2}^{2} \Delta_{2}^{3}$. From Table 4 and Theorem 4.1 it follows that we can choose

$$
\begin{aligned}
\rho_{1} & =\frac{\Delta_{2}^{1} \Delta_{2}^{2}}{\Delta_{1}^{1} \Delta_{1}^{2}+\Delta_{2}^{1} \Delta_{1}^{2}+\Delta_{2}^{1} \Delta_{2}^{2}}, \\
\rho_{2} & =\frac{\Delta_{1}^{1} \Delta_{1}^{2}}{\Delta_{1}^{1} \Delta_{1}^{2}+\Delta_{2}^{1} \Delta_{1}^{2}+\Delta_{2}^{1} \Delta_{2}^{2}}, \\
\rho_{3} & =\frac{\Delta_{2}^{1} \Delta_{1}^{2}}{\Delta_{1}^{1} \Delta_{1}^{2}+\Delta_{2}^{1} \Delta_{1}^{2}+\Delta_{2}^{1} \Delta_{2}^{2}} .
\end{aligned}
$$

Next we consider the case where $\Delta_{1}^{1} \Delta_{1}^{2} \Delta_{1}^{3}<\Delta_{2}^{1} \Delta_{2}^{2} \Delta_{2}^{3}$, i.e. $\sqrt[3]{\alpha_{1} \alpha_{2} \alpha_{3}}<1$ We show that $\rho$ can be chosen such that the conditions of Theorem 4.4.4 as listed in Table 3 are satisfied. We define $\gamma=1 / \sqrt[3]{\alpha_{1} \alpha_{2} \alpha_{3}}>1$ and

$$
\begin{aligned}
\rho_{1} & =\frac{\gamma^{2} \alpha_{2} \alpha_{3}}{1+\gamma \alpha_{3}+\gamma^{2} \alpha_{2} \alpha_{3}} \\
\rho_{2} & =\frac{\gamma \alpha_{3}}{1+\gamma \alpha_{3}+\gamma^{2} \alpha_{2} \alpha_{3}} \\
\rho_{3} & =\frac{1}{1+\gamma \alpha_{3}+\gamma^{2} \alpha_{2} \alpha_{3}} .
\end{aligned}
$$

It therefore holds that

$$
\begin{aligned}
& \frac{\rho_{1}}{\rho_{2}}=\gamma \alpha_{2}>\alpha_{2}, \\
& \frac{\rho_{2}}{\rho_{3}}=\gamma \alpha_{3}>\alpha_{3}, \\
& \frac{\rho_{3}}{\rho_{1}}=\frac{1}{\gamma^{2} \alpha_{2} \alpha_{3}}=\gamma \alpha_{1}>\alpha_{1} .
\end{aligned}
$$

Q.E.D.

If we think of the parameters $\rho_{i}$ as a measure of bargaining power, then Theorem 7.1 makes clear that irrespective of the players' utility functions, delay in bargaining can be avoided under an appropriate distribution of bargaining power. This theorem extends the no-delay result reported earlier for symmetric procedures under three players with compomise-prone utility functions to arbitrary utility functions.

The next result shows that the expected delay in bargaining goes to infinity when one player has all the bargaining power.

THEOREM 7.2 Let $u$ be such that the geometric mean of the players' risk coefficients is less than or equal to one. Consider a sequence of recognition probability vectors $\left(\rho^{n}\right)_{n \in \mathbb{N}}$ which converges to $e^{i}$, the $i$-th unit vector, for some $i=1,2,3$. For $n \in \mathbb{N}$, let $\left(p^{n}, q^{n}\right)$ be a $C P E$ of $\left(u, \rho^{n}\right)$ and denote the corresponding delay probability by $\delta_{n}$. Then $\lim _{n \rightarrow \infty} \delta_{n}=1$.

Proof: Without loss of generality, we may assume that for all $n \in \mathbb{N},\left(u, \rho^{n}\right)$ satisfies the conditions of exactly one of the theorems, i.e. one of the 15 cases displayed in Table 4.

According to Table 4, the lower bound on the delay probability following from an equilibrium of Theorem 4.1 is given by

$$
1-\left(1+\alpha_{2}+\alpha_{1} \alpha_{2}\right) \min \left\{\rho_{1}^{n}, \frac{\rho_{2}^{n}}{\alpha_{1} \alpha_{2}}, \frac{\rho_{3}^{n}}{\alpha_{2}}\right\} .
$$


Clearly, this lower bound converges to 1 when $n \rightarrow \infty$.

According to Table 3, the first line of conditions in Theorem 4.3.1 states that $\rho_{2}^{n} \geq \alpha_{3} \beta_{3}$, so $\lim _{n \rightarrow \infty} \rho_{2}^{n}=1$. Then Table 4, first line corresponding to 4.3.1, yields that $\lim _{n \rightarrow \infty} \delta_{n}=1$. The other cases corresponding to Theorem 4.3.1 follow by symmetry.

According to Table 3, the first line of conditions in Theorem 4.3.2 states that $\rho_{2}^{n}<$ $\alpha_{3} \beta_{3}$, so it is impossible that $\lim _{n \rightarrow \infty} \rho_{2}^{n}=1$, so $\lim _{n \rightarrow \infty} \rho_{2}^{n}=0$. Then Table 4, first line corresponding to 4.3 .2 , yields that $\lim _{n \rightarrow \infty} \delta_{n}=1$. The other cases corresponding to Theorem 4.3.2 follow by symmetry.

It is evident from Table 4 that the delay probability following from Theorem 4.4 .1 goes to 1 when $n$ goes to infinity.

According to Table 3, the first line of conditions in Theorem 4.4.2 states that $\rho_{1}^{n} \leq \alpha_{2} \rho_{2}^{n}$, so $\lim _{n \rightarrow \infty} \rho_{1}^{n}=0$. Then Table 4, first line corresponding to 4.4.2, yields that $\lim _{n \rightarrow \infty} \delta_{n}=1$. The other cases corresponding to Theorem 4.4.2 follow by symmetry.

According to Table 3, the first line of conditions in Theorem 4.4.3 states that $\rho_{1}^{n}<$ $1 /\left(1+\alpha_{1}+\alpha_{1} \alpha_{3}\right)$, so $\lim _{n \rightarrow \infty} \rho_{1}^{n}=0$. Then Table 4, first line corresponding to 4.4.3, yields that the lower bound on the delay probability following from an equilibrium converges to 1. The other cases corresponding to Theorem 4.4 .3 follow by symmetry.

Table 3 demonstrates that $\left(u, \rho^{n}\right)_{n \in \mathbb{N}}$ cannot satisfy the conditions of Theorem 4.4.4. Suppose without loss of generality that $\lim _{n \rightarrow \infty} \rho_{1}^{n}=0$. Since $\rho_{2}^{n}<\rho_{1}^{n} / \alpha_{2}$, we have $\lim _{n \rightarrow \infty} \rho_{2}^{n}=0$. Since $\rho_{3}^{n}<\rho_{2}^{n} / \alpha_{3}$, we have $\lim _{n \rightarrow \infty} \rho_{3}^{n}=0$. It follows that $\rho$ converges to the zero vector, a contradiction.

Q.E.D.

We also analyze whether cycles can occur. Cycles should occur according to the Condorcet logic. Other authors like Chwe (1994) have argued using tools from cooperative game theory that cycles should not occur when players are farsighted. We say that a particular play of the game has resulted in a cycle if all three alternatives have been proposed and rejected, before some alternative is accepted. An equilibrium is said to have a cycle if there is a positive probability that the equilibrium path has resulted in a cycle. If an equilibrium has a cycle, then clearly there is also a positive probability on an equilibrium path where consecutively alternatives $1,2,3$, and 1 are proposed and rejected.

TheOREM 7.3 The set of games $(u, \rho) \in \mathcal{G}$ admitting CPEs with cycles has Lebesgue measure zero.

Proof: It is easily verified that, with the exception of Theorem 4.1 and 4.4.3, there is always an alternative that, in equilibrium, is never rejected when being proposed. The Conditions in Theorem 4.1 and 4.3 are only satisfied for sets of games having a closure with Lebesgue measure zero.

Q.E.D.

Cycles occur with positive probability in the CPEs of Theorem 4.1 and 4.4.3. It is easily verified that for Theorem 4.1 there is always a CPE where the equilibrium path results in a cycle with probability arbitrarily close to one. But the conditions of these theorems hold in degenerate cases only. 


\section{The Role of Risk Aversion}

In this section we investigate the role of risk aversion. We concentrate on the impact of risk aversion on the equilibrium outcome probability vector $\left(\bar{\pi}_{1}, \bar{\pi}_{2}, \bar{\pi}_{3}\right)$. Player 1 is at least as good off with the lottery $\left(\bar{\pi}_{1}^{\prime}, \bar{\pi}_{2}^{\prime}, \bar{\pi}_{3}^{\prime}\right)$ instead of $\left(\bar{\pi}_{1}, \bar{\pi}_{2}, \bar{\pi}_{3}\right)$ when $\bar{\pi}_{1}^{\prime} \geq \bar{\pi}_{1}, \bar{\pi}_{2}^{\prime} \geq \bar{\pi}_{2}$, and $\bar{\pi}_{3}^{\prime} \leq \bar{\pi}_{3}$.

The standard approach to risk aversion in bargaining is to take a concave transformation of one of the player's utility functions. Taking such a transformation for player $i$ will reduce his risk coefficient, because the gap between $u_{m_{i}}^{i}$ and $u_{w_{i}}^{i}$ will become relatively larger than the gap between $u_{b_{i}}^{i}$ and $u_{m_{i}}^{i}$. The equilibrium probability vector $\left(\bar{\pi}_{1}, \bar{\pi}_{2}, \bar{\pi}_{3}\right)$ can be rewritten in terms of risk coefficients only and for that reason we perform the comparative statics with respect to changes in risk aversion as changes in the player's risk coefficients.

Table 5 reports the equilibrium outcome probability vectors. For Theorem 4.4.3, first line, $\lambda \geq 1$ should be chosen to satisfy (4.66) and (4.67). For the other two lines corresponding to Theorem 4.4.3, $\lambda \geq 1$ should satisfy the appropriate analogues of (4.66) and (4.67). We evaluate the local effects of a change in a player's risk coefficient for the non-degenerate cases.

\begin{tabular}{|c|c|c|c|c|c|c|c|}
\hline Theorem & & Utilities & & $\bar{\pi}_{1}$ & $\bar{\pi}_{2}$ & $\bar{\pi}_{3}$ & Occurrence \\
\hline 4.1 & $\overline{z^{1}}=u_{2}^{1}$ & $\bar{z}^{2}=u_{3}^{2}$ & $\bar{z}^{3}=u_{1}^{3}$ & $\frac{\alpha_{2}}{\frac{\alpha_{2}}{1+\alpha_{2}+\alpha_{1} \alpha_{0}}}$ & $\frac{1}{1+\alpha_{0}+\alpha_{1} \alpha_{0}}$ & $\frac{\alpha_{1} \alpha_{2}}{1+\alpha_{2}+\alpha_{1} \alpha_{2}}$ & Degenerate \\
\hline 4.3 .1 & $\begin{array}{l}\bar{z}^{1}=u_{2}^{1} \\
\bar{z}^{1}=u_{2}^{1} \\
\bar{z}^{1}<u_{2}^{1}\end{array}$ & $\begin{array}{l}\bar{z}^{2}=u_{3}^{2} \\
\bar{z}^{2}<u_{3}^{2} \\
\bar{z}^{2}=u_{3}^{2}\end{array}$ & $\begin{array}{l}\bar{z}^{3}<u_{1}^{3} \\
\bar{z}^{3}=u_{1}^{3} \\
\bar{z}^{3}=u_{1}^{3}\end{array}$ & $\begin{array}{l}\frac{\alpha_{2}}{1+\alpha_{2}+\alpha_{1} \alpha_{2}} \\
\frac{1}{1+\alpha_{1}+\alpha_{1} \alpha_{3}} \\
\frac{\alpha_{2} \alpha_{3}}{1+\alpha_{2}+\alpha_{2} \alpha_{3}}\end{array}$ & $\begin{array}{c}\frac{1}{1+\alpha_{2}+\alpha_{1} \alpha_{2}} \\
\frac{\alpha_{1} \alpha_{3}}{1+\alpha_{1}+\alpha_{1} \alpha_{3}} \\
\frac{\alpha_{3}}{1+\alpha_{2}+\alpha_{2} \alpha_{2}}\end{array}$ & $\begin{array}{l}\frac{\alpha_{1} \alpha_{2}}{1+\alpha_{2}+\alpha_{1} \alpha_{2}} \\
\frac{\alpha_{1}}{1+\alpha_{1}+\alpha_{1} \alpha_{3}} \\
\frac{1}{1+\alpha_{2}+\alpha_{2} \alpha_{3}}\end{array}$ & \\
\hline 4.3 .2 & $\begin{array}{l}\bar{z}^{1}<u_{2}^{1} \\
\bar{z}^{1}=u_{2}^{1} \\
\bar{z}^{1}<u_{2}^{1}\end{array}$ & $\begin{array}{l}\bar{z}^{2}=u_{3}^{2} \\
\bar{z}^{2}<u_{3}^{2} \\
\bar{z}^{2}<u_{3}^{2}\end{array}$ & $\begin{array}{l}\bar{z}^{3}<u_{1}^{3} \\
\bar{z}^{3}<u_{1}^{3} \\
\bar{z}^{3}=u_{1}^{3}\end{array}$ & $\begin{array}{l}\frac{\alpha_{2}\left(\alpha_{3}+\rho_{2}\right)}{1+\alpha_{3}+\alpha_{2} \alpha_{3}} \\
\frac{\alpha_{2}+\rho_{1}}{1+\alpha_{2}+\alpha_{1} \alpha_{2}} \\
\frac{1-\rho_{3}\left(1+\alpha_{3}\right)}{1+\alpha_{1}+\alpha_{1} \alpha_{3}}\end{array}$ & $\begin{array}{l}\frac{\alpha_{3}+\rho_{2}}{1+\alpha_{3}+\alpha_{2} \alpha_{3}} \\
\frac{1-\rho_{1}\left(1+\alpha_{1}\right)}{1+\alpha_{2}+\alpha_{1} \alpha_{2}} \\
\frac{\alpha_{3}\left(\alpha_{1}+\rho_{3}\right)}{1+\alpha_{1}+\alpha_{1} \alpha_{3}}\end{array}$ & $\begin{array}{l}\frac{1-\rho_{2}\left(1+\alpha_{2}\right)}{1+\alpha_{3}+\alpha_{2} \alpha_{3}} \\
\frac{\alpha_{1}\left(\alpha_{2}+\rho_{1}\right)}{1+\alpha_{2}+\alpha_{1} \alpha_{2}} \\
\frac{\alpha_{1}+\rho_{3}}{1+\alpha_{1}+\alpha_{1} \alpha_{2}}\end{array}$ & \\
\hline 4.4 .1 & $\bar{z}^{1}=u_{2}^{1}$ & $\bar{z}^{2}=u_{3}^{2}$ & $\bar{z}^{3}=u_{1}^{3}$ & $\frac{\alpha_{2}}{1+\alpha_{2}+\alpha_{1} \alpha_{2}}$ & $\frac{1}{1+\alpha_{2}+\alpha_{1} \alpha_{2}}$ & $\frac{\alpha_{1} \alpha_{2}}{1+\alpha_{2}+\alpha_{1} \alpha_{2}}$ & Degenerate \\
\hline 4.4 .2 & $\begin{array}{l}\bar{z}^{1}=u_{2}^{1} \\
\bar{z}^{1}=u_{2}^{1} \\
\bar{z}^{1}<u_{2}^{1}\end{array}$ & $\begin{array}{l}\bar{z}^{2}=u_{3}^{2} \\
\bar{z}^{2}<u_{3}^{2} \\
\bar{z}^{2}=u_{3}^{2}\end{array}$ & $\begin{array}{l}\bar{z}^{3}<u_{1}^{3} \\
\bar{z}^{3}=u_{1}^{3} \\
\bar{z}^{3}=u_{1}^{3}\end{array}$ & $\begin{array}{l}\frac{1+\alpha_{2}+\alpha_{1} \alpha_{2}}{\alpha_{2}} \frac{1+\alpha_{2}+\alpha_{1} \alpha_{2}}{1} \frac{1}{1+\alpha_{1}+\alpha_{1} \alpha_{3}} \\
\frac{\alpha_{2} \alpha_{3}}{1+\alpha_{3}+\alpha_{2} \alpha_{3}}\end{array}$ & $\begin{array}{l}\frac{1}{1+\alpha_{2}+\alpha_{1} \alpha_{2}} \\
\frac{\alpha_{1} \alpha_{3}}{1+\alpha_{1}+\alpha_{1} \alpha_{3}} \\
\frac{\alpha_{3}}{1+\alpha_{3}+\alpha_{2} \alpha_{3}}\end{array}$ & $\begin{array}{l}\frac{1+\alpha_{2}+\alpha_{1} \alpha_{2}}{\alpha_{1} \alpha_{2}} \frac{1+\alpha_{2}+\alpha_{1} \alpha_{2}}{\frac{\alpha_{1}}{1+\alpha_{1}+\alpha_{1} \alpha_{3}}} \frac{1}{1+\alpha_{3}+\alpha_{2} \alpha_{3}}\end{array}$ & \\
\hline 4.4 .3 & $\begin{array}{l}\bar{z}^{1}=u_{2}^{1} \\
\bar{z}^{1}<u_{2}^{1} \\
\bar{z}^{1}<u_{2}^{1}\end{array}$ & $\begin{array}{l}\bar{z}^{2}<u_{3}^{2} \\
\bar{z}^{2}=u_{3}^{2} \\
\bar{z}^{2}<u_{3}^{2}\end{array}$ & $\begin{array}{l}\bar{z}^{3}<u_{1}^{3} \\
\bar{z}^{3}<u_{1}^{3} \\
\bar{z}^{3}=u_{1}^{3}\end{array}$ & $\begin{array}{c}\lambda \rho_{1} \\
\alpha_{2} \lambda \rho_{2} \\
1-\lambda \rho_{3}\left(1+\alpha_{3}\right)\end{array}$ & $\begin{array}{c}1-\lambda \rho_{1}\left(1+\alpha_{1}\right) \\
\lambda \rho_{2} \\
\alpha_{3} \lambda \rho_{3}\end{array}$ & $\begin{array}{c}\alpha_{1} \lambda \rho_{1} \\
1-\lambda \rho_{2}\left(1+\alpha_{2}\right) \\
\lambda \rho_{3}\end{array}$ & $\begin{array}{l}\text { Degenerate } \\
\text { Degenerate } \\
\text { Degenerate }\end{array}$ \\
\hline 4.4 .4 & $\bar{z}^{1}<u_{2}^{1}$ & $\bar{z}^{2}<u_{3}^{2}$ & $\bar{z}^{3}<u_{1}^{3}$ & $\rho_{1}$ & $\rho_{2}$ & $\rho_{3}$ & \\
\hline
\end{tabular}

Table 5: CPE outcome probabilities.

Consider the first line of Theorem 4.3.1 and 4.4.2 for which

$$
\left(\bar{\pi}_{1}, \bar{\pi}_{2}, \bar{\pi}_{3}\right)=\left(\frac{\alpha_{2}}{1+\alpha_{2}+\alpha_{1} \alpha_{2}}, \frac{1}{1+\alpha_{2}+\alpha_{1} \alpha_{2}}, \frac{\alpha_{1} \alpha_{2}}{1+\alpha_{2}+\alpha_{1} \alpha_{2}}\right) .
$$

We have that

$$
\begin{array}{lll}
\frac{\partial \bar{\pi}_{1}}{\partial \alpha_{1}}<0, & \frac{\partial \bar{\pi}_{2}}{\partial \alpha_{1}}<0, & \frac{\partial \bar{\pi}_{3}}{\partial \alpha_{1}}>0, \\
\frac{\partial \bar{\pi}_{1}}{\partial \alpha_{2}}>0, & \frac{\partial \bar{\pi}_{2}}{\partial \alpha_{2}}<0, & \frac{\partial \bar{\pi}_{3}}{\partial \alpha_{2}}>0, \\
\frac{\partial \bar{\pi}_{1}}{\partial \alpha_{3}}=0, & \frac{\partial \bar{\pi}_{2}}{\partial \alpha_{3}}=0, & \frac{\partial \bar{\pi}_{3}}{\partial \alpha_{3}}=0,
\end{array}
$$


and $\frac{\partial \bar{\pi}_{1}}{\partial \alpha_{i}}+\frac{\partial \bar{\pi}_{2}}{\partial \alpha_{i}}+\frac{\partial \bar{\pi}_{3}}{\partial \alpha_{i}}=0$ for $i=1,2,3$.

An increase in $\alpha_{1}$ yields player 1 lower probabilities for obtaining his best and middle alternatives and an increased probability for obtaining his worst alternative. So, a less risk averse type of player 1 is unambiguously worse off. The next question is whether one or both other players benefit from such a change. This issue is straightforward for player 3, who would face an increased probability for obtaining his best alternative and lower probabilities for obtaining his middle and worst alternatives. Therefore, player 3 is unambiguously better off if player 1 becomes less risk averse. Player 2 faces lower probabilities for both his best and worst alternatives and an increased probability for his middle alternative. Notice however that player 2 obtains an equilibrium utility of $\bar{z}^{2}=u_{3}^{2}$, which is not affected by the risk coefficient of player 1 .

Next, an increase in $\alpha_{2}$ yields player 2 a lower probability for obtaining his best alternative and increased probabilities for attaining his middle and worst alternatives. A less risk averse type of player 2 is unambiguously worse off, albeit that player 2 is affected in a different manner than player 1 is affected by changes in $\alpha_{1}$. Since player 3 faces increased probabilities for obtaining his best and middle alternatives and a lower probability for obtaining his worst alternative, player 3 is unambiguously better off if player 2 becomes less risk averse. The equilibrium utility for player 1 is not affected by the risk coefficient of player 3 .

Finally, changes in player 3's risk coefficient have no effect on the outcome probability vector, so both situations are unambiguously equivalent for all the players.

The second and third line corresponding to Theorem 4.3 .1 and 4.4 .2 are obtained by a permutation of the players and lead to analogous results.

Consider the first line corresponding to Theorem 4.3.2 for which

$$
\left(\bar{\pi}_{1}, \bar{\pi}_{2}, \bar{\pi}_{3}\right)=\left(\frac{\alpha_{2}\left(\alpha_{3}+\rho_{2}\right)}{1+\alpha_{3}+\alpha_{2} \alpha_{3}}, \frac{\alpha_{3}+\rho_{2}}{1+\alpha_{3}+\alpha_{2} \alpha_{3}}, \frac{1-\rho_{2}\left(1+\alpha_{2}\right)}{1+\alpha_{3}+\alpha_{2} \alpha_{3}}\right) .
$$

We have that

$$
\begin{array}{lll}
\frac{\partial \bar{\pi}_{1}}{\partial \alpha_{1}}=0, & \frac{\partial \bar{\pi}_{2}}{\partial \alpha_{1}}=0, & \frac{\partial \bar{\pi}_{3}}{\partial \alpha_{1}}=0, \\
\frac{\partial \bar{\pi}_{1}}{\partial \alpha_{2}}>0, & \frac{\partial \bar{\pi}_{2}}{\partial \alpha_{2}}<0, & \frac{\partial \bar{\pi}_{3}}{\partial \alpha_{2}}<0, \\
\frac{\partial \pi_{1}}{\partial \alpha_{3}}>0, & \frac{\partial \bar{\pi}_{2}}{\partial \alpha_{3}}>0, & \frac{\partial \bar{\pi}_{3}}{\partial \alpha_{3}}<0,
\end{array}
$$

and $\frac{\partial \bar{\pi}_{1}}{\partial \alpha_{1}}+\frac{\partial \bar{\pi}_{2}}{\partial \alpha_{1}}+\frac{\partial \bar{\pi}_{3}}{\partial \alpha_{1}}=0$ for $i=1,2,3$. For this derivation, observe that

$$
\frac{\partial}{\partial \alpha_{3}} \frac{\alpha_{2}\left(\alpha_{3}+\rho_{2}\right)}{1+\alpha_{3}+\alpha_{2} \alpha_{3}}=\frac{\alpha_{2}\left(1-\rho_{2}\left(1+\alpha_{2}\right)\right)}{\left(1+\alpha_{3}+\alpha_{2} \alpha_{3}\right)^{2}}=\frac{\alpha_{2}}{1+\alpha_{3}+\alpha_{2} \alpha_{3}} \bar{\pi}_{3}>0 .
$$

First, changes in player 1's risk coefficient have no effect. Second, in case player 2 becomes less risk averse then this player is always worse off and player 1 is always better off. The marginal effect of a change in $\alpha_{2}$ on the equilibrium utility of player 3 is given by

$$
\begin{aligned}
\frac{\partial \pi_{1}}{\partial \alpha_{2}} u_{1}^{3}+\frac{\partial \pi_{2}}{\partial \alpha_{2}} u_{2}^{3}+\frac{\partial \pi_{3}}{\partial \alpha_{3}} u_{3}^{3} & =\frac{\left(\alpha_{3}+\rho_{2}\right)\left(1+\alpha_{3}\right)}{\left(1+\alpha_{3}+\alpha_{2} \alpha_{3}\right)^{2}} u_{1}^{3}-\frac{\left(\alpha_{3}+\rho_{2}\right) \alpha_{3}}{\left(1+\alpha_{3}+\alpha_{2} \alpha_{3}\right)^{2}} u_{2}^{3}-\frac{\alpha_{3}+\rho_{2}}{\left(1+\alpha_{3}+\alpha_{2} \alpha_{3}\right)^{2}} u_{3}^{3} \\
& =\frac{\left(\alpha_{3}+\rho_{2}\right) \alpha_{3}}{\left(1+\alpha_{3}+\alpha_{2} \alpha_{3}\right)^{2}} \Delta_{2}^{3}-\frac{\alpha_{3}+\rho_{2}}{\left(1+\alpha_{3}+\alpha_{2} \alpha_{3}\right)^{2}} \Delta_{1}^{3}=0 .
\end{aligned}
$$

Third, in case player 3 becomes less risk averse then this player is always worse off and player 1 is always better off. The marginal effect of a change in $\alpha_{3}$ on the equilibrium 
utility of player 2 is given by

$$
\begin{aligned}
\frac{\partial \pi_{1}}{\partial \alpha_{3}} u_{1}^{2}+\frac{\partial \pi_{2}}{\partial \alpha_{3}} u_{2}^{2}+\frac{\partial \pi_{3}}{\partial \alpha_{3}} u_{3}^{2}=\frac{\alpha_{2}\left(1-\rho_{2}\left(1+\alpha_{2}\right)\right)}{\left(1+\alpha_{3}+\alpha_{2} \alpha_{3}\right)^{2}} u_{1}^{2}+\frac{\left(1-\rho_{2}\left(1+\alpha_{2}\right)\right)}{\left(1+\alpha_{3}+\alpha_{2} \alpha_{3}\right)^{2}} u_{2}^{2}-\frac{\left(1-\rho_{2}\left(1+\alpha_{2}\right)\right)\left(1+\alpha_{2}\right)}{\left(1+\alpha_{3}+\alpha_{2} \alpha_{3}\right)^{2}} u_{3}^{2} \\
=-\frac{\alpha_{2}\left(1-\rho_{2}\left(1+\alpha_{2}\right)\right)}{\left(1+\alpha_{3}+\alpha_{2} \alpha_{3}\right)^{2}} \Delta_{2}^{2}+\frac{1-\rho_{2}\left(1+\alpha_{2}\right)}{\left(1+\alpha_{3}+\alpha_{2} \alpha_{3}\right)^{2}} \Delta_{1}^{2}=0 .
\end{aligned}
$$

The second and third line corresponding to Theorem 4.3.2 are obtained by a permutation of the players and lead to analogous results.

Consider Theorem 4.4 .4 for which $\left(\bar{\pi}_{1}, \bar{\pi}_{2}, \bar{\pi}_{3}\right)=\left(\rho_{1}, \rho_{2}, \rho_{3}\right)$. Then, $\frac{\partial \bar{\pi}_{i}}{\partial \alpha_{j}}=0$ for all $i, j=1,2,3$. This implies that changes in any player's risk aversion have no effect on the outcome probability vector and for each player the situation before and after such a change is unambiguously equivalent.

In summary, we obtain effects that are opposite to the well-known effects from two player alternating-offers bargaining and the Nash bargaining solution, see e.g. Roth (1985), Safra, Zhou, and Zilcha (1990), and Kihlstrom, Roth, and Schmeidler (1991). In these references, less risk aversion leads to a better bargaining result. Here, for the three cases of Theorem 4.3.1, 4.3.2, and 4.4.2, we obtain that the opposite effect that less risk aversion leads to a worse outcome in the sense that the probability of attaining the best alternative decreases while the probabilities for obtaining the middle and worst alternative increase. In the remaining main case, the one of Theorem 4.4.4, changes in risk attitudes have no effect. So, combining these four cases, we find opposite effects for risk aversion on the own bargaining position. Due to Pareto efficiency of every CPE, at least one player has to benefit. We find for the main cases that at least one player is unaffected by a change in one of the risk coefficients.

\section{Concluding Remarks}

We model decision making by three players over a set consisting of three alternatives as a bargaining game in extensive form. The set-up corresponds to the well-known Condorcet paradox in the sense that the players' utility functions are such that naive majority voting over the alternatives results in a Condorcet cycle. We consider the case of sophisticated players and formulate a strategic bargaining model to study the proposals and acceptance/rejection decisions as made by rational players.

The paper analyzes the consistent subgame perfect equilibria of the game. It is shown that such equilibria exist if and only if the geometric mean of the players' risk coefficients is less than or equal to 1 . A sufficient condition for equilibrium existence is that players be compromise-prone, i.e. the utility of the middle alternative is at least as high as the utility of getting the best alternative with probability one half and getting the worst alternative with probability one half. These conditions also admit an interpretation in terms of the risk limit of Zeuthen (1930) and Harsanyi (1977).

When the geometric mean of the players' risk coefficients is less than or equal to 1 , equilibria do not only exist, but are also typically unique. A player never proposes his worst alternative and knows that his middle alternative, when proposed, will be accepted for sure by the player for whom this is the best alternative. The trade-off for a proposer is therefore to propose either his best alternative, which might be rejected, or to propose his middle alternative which is accepted for sure. A player that has to respond to a proposal accepts his best alternative and rejects his worst alternative. He faces a trade-off when 
his middle alternative is offered to him. Accepting this alternative leads to utility equal to the middle alternative for sure, rejecting it leads to a particular probability distribution over the three alternatives. The situation where an agreement is never reached cannot be supported as an equilibrium phenomenon.

Many equilibria feature delay before an agreement is reached. We show that for any specification of the players' risk coefficients, there are recognition probability vectors for which no delay occurs before an agreement is reached, but also that expected delay goes to infinity when recognition probability vectors converge to a unit vector, i.e. in the limit a single player is the only proposer. Contrary to the usual intuition, decreasing risk aversion weakens the bargaining position of a player.

In this paper, we study the case without discounting. The question how the situation with little discounting is related to the case without discounting is studied in the companion paper Herings and Houba (2010). In this reference, also natural extensions of the model with more than three alternatives and more than three players are dealt with. Though the calculations of the three alternative-three player case are quite demanding, the end results are sufficiently neat to sustain some hope that progress on the general case with Condorcet cycles is within reach.

\section{References}

Banks, J., and J. Duggan (2000), "A Bargaining Model of Collective Choice," American Political Science Review, 94, 73-88.

Baron, D.P., and J.A. Ferejohn (1989), "Bargaining in Legislatures," American Political Science Review, 83, 1181-1206.

Bhaskar, V., G.J. Mailath, and S. Morris (2009), "A Foundation for Markov Equilibria in Infinite Horizon Perfect Information Games," PIER Working Paper 09-029, $1-21$.

Binmore, K. (1987), "Perfect Equilibria in Bargaining Models," in K. Binmore and P. Dasgupta (eds.), The Economics of Bargaining, Basil Blackwell, Oxford, UK, pp. ry-105.

Bloch, F. (1996), "Sequential Formation of Coalitions in Games with Externalities and Fixed Payoff Division," Games and Economic Behavior, 14, 90-123.

Breton, M. Le (1987), "On the Core of Voting Games," Social Choice and Welfare, 4, 295-305.

Chatterjee, K., B. Dutta, D. Ray, and K. Sengupta (1993), "A Noncooperative Theory of Coalitional Bargaining," Review of Economic Studies, 60, 463-477.

Chwe, M. S.-Y. (1994), "Farsighted Coalitional Stability," Journal of Economic Theory, 63, 299-325.

Cox, G.W. (1984), "Non-Collegial Simple Games and the Nowhere Denseness of the Set of Preference Profiles Having a Core," Social Choice and Welfare, 1, 159-164. 
Fink, A.M. (1964), "Equilibrium in a Stochastic n-Person Game," Journal of Science of Hiroshima University Series A-I, 28, 89-93.

Haller, H., and R. Lagunoff (2000), "Genericity and Markovian Behavior in Stochastic Games," Econometrica, 68, 1231-1248.

Harsanyi, J.C. (1977), Rational Behavior and Bargaining Equilibrium in Games and Social Situations, Cambridge University Press, Cambridge.

Harsanyi, J.C., and R. Selten (1988), A General Theory of Equilibrium Selection in Games, MIT Press, Cambridge.

Herings, P.J.J., and H. Houba (2010), "Discrete Sets of Alternatives and Costless Delay in Negotiations," Working Paper.

Herings, P.J.J., and R.J.A.P. Peeters (2004), "Stationary Equilibria in Stochastic Games: Structure, Selection, and Computation," Journal of Economic Theory, 118, 32-60.

Houba, H. (2008), "On continuous-time Markov processes in bargaining," Economic Letters 100, 280-283.

Kihlstrom, R., A. Roth, and D. Schmeidler (1991), "Risk Aversion and Solutions to Nash's Bargaining Problem," in O. Moeschlin and D. Pallaschke (eds), Game Theoretic and Mathematical Economics, Amsterdam, North-Holland, 34-47.

Maskin, E.S., and J. Tirole (2001), "Markov Perfect Equilibrium, I. Observable Actions," Journal of Economic Theory, 100, 191-219.

McKelvey, R.D. (1976), "Intransitivities in Multidimensional Voting Models and Some Implications for Agenda Control," Journal of Economic Theory, 12, 472-482.

McKelvey, R.D. (1979), "General Conditions for Global Intransitivities in Formal Voting Models," Econometrica, 47, 1085-1112.

Plott, C.R. (1967), "A Notion of Equilibrium and its Possibility Under Majority Rule," American Economic Review, 57, 787-806.

Roth, A. (1985), "A Note on Risk Aversion in a Perfect Equilibrium Model of Bargaining," Econometrica, 53, 207-211.

Rubinstein, A. (1979), "A Note about the "Nowhere Denseness" of Societies Having an Equilibrium under Majority Rule," Econometrica, 47, 511-514.

Rubinstein, A. (1982), "Perfect Equilibrium in a Bargaining Model," Econometrica, 50, 97-109.

Safra, Z., L. Zhou, and I. Zilcha (1990), "Risk Aversion in the Nash Bargaining Problem with Risky Outcomes and Risky Disagreement Points," Econometrica, 58, 961965. 
Schofield, N. (1983), "Generic Instability of Majority Rule," Review of Economic Studies, 50, 695-705.

Sobel, M.J. (1971), "Noncooperative Stochastic Games," The Annals of Mathematical Studies, 42, 1930-1935.

Takahashi M. (1964), "Equilibrium points of stochastic, noncooperative n-person games," Journal of Science of Hiroshima University Series A-I, 28, 95-99.

Zeuthen, F. (1930), Problems of Monopoly and Economic Welfare, Routledge, London. 